\title{
A spectroscopic atlas of post-AGB stars and planetary nebulae selected from the IRAS point source catalogue ${ }^{\star, \star \star}$
}

\author{
O. Suárez ${ }^{1}$, P. García-Lario ${ }^{2}$, A. Manchado ${ }^{3,4}$, M. Manteiga ${ }^{5}$, A. Ulla ${ }^{6}$, and S. R. Pottasch ${ }^{7}$ \\ 1 Laboratorio de Astrofísica Espacial y Física Fundamental, INTA, Apartado de Correos 50727, 28080 Madrid, Spain \\ e-mail: olga@laeff.inta.es \\ 2 Research and Scientific Support Department of ESA, European Space Astronomy Centre, Villafranca del Castillo, \\ Apartado de Correos 50727, 28080 Madrid, Spain \\ Instituto de Astrofísica de Canarias, c/via Lactea, s/n, 38200 La Laguna, Tenerife, Spain \\ ${ }^{4}$ Consejo Superior de Investigaciones Científicas (CSIC), Spain \\ 5 Departamento de Ciencias de la Navegación y de la Tierra, E.T.S. de Náutica y Máquinas, Universidade da Coruña, \\ 15011 A Coruña, Spain \\ ${ }^{6}$ Departamento de Física Aplicada, Facultade de Ciencias do Mar, Campus Marcosende-Lagoas, Universidade de Vigo, \\ 36310 Vigo, Pontevedra, Spain \\ 7 Kapteyn Astronomical Institute, Postbus 800, 9700 AV Groningen, The Netherlands
}

Received 26 August 2005 / Accepted 5 July 2006

\section{ABSTRACT}

\begin{abstract}
Aims. We study the optical spectral properties of a sample of stars showing far infrared colours similar to those of well-known planetary nebulae. The large majority of them were unidentified sources or poorly known in the literature at the time when this spectroscopic survey started, some 15 years ago.

Methods. We present low-resolution optical spectroscopy, finding charts and improved astrometric coordinates of a sample of 253 IRAS sources.

Results. We have identified 103 sources as post-AGB stars, 21 as "transition sources", and 36 as planetary nebulae, some of them strongly reddened. Among the rest of sources in the sample, we were also able to identify 38 young stellar objects, 5 peculiar stars, and 2 Seyfert galaxies. Up to 49 sources in our spectroscopic sample do not show any optical counterpart, and most of them are suggested to be heavily obscured post-AGB stars, rapidly evolving on their way to becoming planetary nebulae.

Conclusions. An analysis of the galactic distribution of the sources identified as evolved stars in the sample is presented together with a study of the distribution of these stars in the IRAS two-colour diagram. Finally, the spectral type distribution and other properties of the sources identified as post-AGB in this spectroscopic survey are discussed in the framework of stellar evolution.
\end{abstract}

Key words. planetary nebulae: general - stars: AGB and post-AGB - stars: mass-loss - atlases

\section{Introduction}

Post-Asymptotic Giant Branch (post-AGB, hereafter) stars are rapidly evolving low- and intermediate-mass stars $\left(1-8 M_{\odot}\right)$ in the transition phase from the AGB to the Planetary Nebula (PNe, hereafter) stage (Kwok 1993; Habing 1996; van Winckel 2003). Their precursors, the AGB stars, are pulsating stars, very bright in the infrared, which can become heavily obscured in the optical by thick circumstellar envelopes formed as a consequence of their strong mass loss (up to $10^{-4} M_{\odot} / \mathrm{yr}$ ). When the mass loss stops, the AGB star enters the so-called post-AGB stage, which is also accompanied by the cessation of the stellar pulsations. This is followed by a decrease in the optical depth of the circumstellar envelope as a consequence of the expansion, which implies that the central star can be seen again in the optical range if it were ever obscured at the end of the AGB. During this process, the effective temperature of the central star increases. This leads to a rapid change in the spectral type, which migrates from late- to

\footnotetext{
^ Based mainly on observations collected at the European Southern Observatory (La Silla, Chile) and at the Observatorio del Roque de los Muchachos (La Palma, Spain).

$\star \star$ Appendices and Tables 3-6 are only available in electronic form at http://www . aanda.org
}

early-type in very short timescales of just a few thousand years (Vassiliadis \& Wood 1994).

The terminology used to define the various stages preceding the formation of a PN is sometimes confusing. In this work the term "post-AGB star" will be applied to those sources that have already left the strong mass-losing AGB phase. When the temperature of the central star is hot enough $(T \geq 20000 \mathrm{~K})$, the ionization of the envelope starts, and we consider that the star has entered the PN stage. We will adopt the term "transition source" for those stars in an intermediate stage between the post-AGB and PN stages, whose spectra are characterised by the simultaneous detection of a prominent stellar continuum and shock-excited emission lines.

The initial goal of the observations presented here was the discovery of new PNe among the 1084 sources included in the so-called "GLMP catalogue" (García-Lario 1992). This was a colour-selected sample of IRAS sources showing the characteristic far-infrared colours of well known PNe. The strategy was based on the fact that $\mathrm{PNe}$ are located in a well-defined region of the IRAS two-colour diagram [12]-[25] vs. [25]-[60] almost exclusively populated by $\mathrm{PNe}$ and their immediate precursors, the post-AGB stars. In this region, only a small overlap exists with some young stellar objects and a few Seyfert galaxies, while 
normal stars and galaxies show completely different far-infrared colours (Pottasch et al. 1988). Not unexpectedly, as a byproduct of our search for new PNe, we found that many of the observed stars were actually post-AGB stars and transition sources, as well as peculiar very young $\mathrm{PNe}$, rather than genuine, classical PNe.

Indeed, the use of IRAS data proved to be a highly successful method to identify new candidate sources in the transition from the AGB to the PN stage. Their strong infrared excess makes them very bright in the infrared and easily detectable at these wavelengths. Based on their characteristic colours, several lists with potential candidates were compiled in the past by Preite-Martinez (1988), Pottasch et al. (1988), van der Veen et al. (1989), Ratag et al. (1990), Hu et al. (1993), and other authors reported in the Strasbourg-ESO Catalogue of Galactic PNe (Acker et al. 1992). More specific searches for PNe based on other observational criteria were also made by van de Steene \& Pottasch (1993) and Jacoby \& van de Steene (2004). Other searches for post-AGB stars were based on the detection of infrared excesses around stars with optically bright counterparts, like those performed by Oudmaijer et al. (1992) and Oudmaijer (1996) in the last decade. Unfortunately, in many cases no spectroscopic confirmation was ever provided in the literature about the nature of the newly discovered sources. One of the main reasons for this is the poor astrometric accuracy of the IRAS data ( 15-30 arcsec), which makes the identification of the optical counterparts of the selected IRAS sources very difficult, especially in crowded fields close to the galactic plane and/or in the direction of the Galactic Centre.

In this paper we present, for the first time, the whole optical spectroscopic database of stars resulting from the survey carried out by our group in the optical from 1988 to 2003, concentrated on the stars in the GLMP sample for which indications of the presence of an optical counterpart were available. At the time when the observations were performed, priority was given to those sources suspected to be evolved stars. The results are presented in the form of an atlas including the largest set of spectroscopic data on post-AGB stars and transition sources published up to now. The atlas also contains improved astrometric coordinates and finding charts for all the sources observed. The spectral energy distributions (SEDs) from 1 to $100 \mu \mathrm{m}$ of the objects observed were also studied, but they will be presented elsewhere, together with a more detailed analysis of some of the new sources found. Several papers dealing with other individual sources of strong interest found during the survey have already been published (e.g., Manchado et al. 1989b; García Lario et al. 1991; Bobrowsky et al. 1998; García-Lario et al. 1999a)

In Sect. 2 we explain the selection criteria applied to include an IRAS source in our sample. In Sect. 3 we present the observations performed and the data reduction process. Section 4 describes the identification of the sources in the sample and the way the classification of the optical spectra was performed. The main properties of the sources identified as evolved stars are discussed in Sect. 5. Finally, in Sect. 6 we present our conclusions. Spectra and finding charts for individual sources are provided in Appendices A-F (only available in electronic format).

\section{Selection criteria}

The selection criteria applied in this work are essentially the same as those adopted by Pottasch et al. (1988), with some modifications:

(i) The source must be well detected at 12,25 , and $60 \mu \mathrm{m}$ in the IRAS Point Source Catalogue (see Beichman et al. 1988).
The flux quality for each band must be:

$$
\begin{aligned}
& F Q U A L(12 \mu \mathrm{m}) \geq 2 ; \\
& F Q U A L(25 \mu \mathrm{m})=3 ; \\
& F Q U A L(60 \mu \mathrm{m})=3 .
\end{aligned}
$$

(ii) The ratios between the IRAS photometric fluxes must satisfy the following conditions:

$$
\begin{aligned}
& \frac{F_{v}(12 \mu \mathrm{m})}{F_{v}(25 \mu \mathrm{m})} \leq 0.50 ; \\
& \frac{F_{v}(25 \mu \mathrm{m})}{F_{v}(60 \mu \mathrm{m})} \geq 0.35 .
\end{aligned}
$$

(iii) When data at $100 \mu \mathrm{m}$ are of good quality (FQUAL $(100 \mu \mathrm{m})=3)$, we further impose:

$$
\frac{F_{v}(60 \mu \mathrm{m})}{F_{v}(100 \mu \mathrm{m})} \geq 0.60 \text {. }
$$

(iv) Sources must show a low IRAS variability index:

$$
V A R \leq 60 \% .
$$

The choice of criteria (i) and (ii) was mainly a direct consequence of the range of dust temperatures $\left(T_{\mathrm{d}}\right)$ expected in the circumstellar shells of post-AGB stars. If we assume a typical luminosity for a post-AGB star of $L=10^{4} L_{\odot}$, the dust temperature in the shell, following Scoville \& Kwan (1976), would be:

$$
T_{\mathrm{d}}=1.658 f^{-1 / 5} r^{2 / 5} L_{*}^{1 / 5},
$$

where $f$ is the value of the emissivity of the dust grains at $50 \mu \mathrm{m}$, $r$ is the radius of the shell in pc, and $L_{*}$ is the luminosity of the central star in solar units.

Using $f(50 \mu \mathrm{m})=0.004$ (Draine \& Lee 1984), we find a $T_{\mathrm{d}}$ between $200 \mathrm{~K}$ and $80 \mathrm{~K}$ for $r$ between $0.01 \mathrm{pc}$ and $0.1 \mathrm{pc}$, respectively (the expected range of radii of the expanding circumstellar envelope).

Criterium (iii) was added to avoid the potential contamination by young stellar objects, ultra-compact H II regions, and Seyfert galaxies, which can sometimes show very similar colours up to $60 \mu \mathrm{m}$.

Finally, the low IRAS variability was imposed to exclude AGB stars from the sample, since they are known to be strongly variable stars.

All the sources in the original GLMP sample satisfy criteria (i), (ii), and (iii), but not necessarily the last one, concerning the variability. Moreover, in the original GLMP catalogue, about half of the entries were associated with objects that were well identified in the literature, most of them PNe, and they were excluded from the atlas presented in this paper. There was also a significant number of known young stellar objects $(\sim 20 \%)$ and a few Seyfert galaxies $(\sim 5 \%)$, which were not considered either.

A slight, more recent modification of the selection criteria allowed the inclusion of about 100 additional sources in an extended version of the GLMP catalogue (García-Lario, private communication). This extension also contains those IRAS sources not detected in the $12 \mu \mathrm{m}$ IRAS band (FQUAL $(12 \mu \mathrm{m})=1)$, but satisfying the rest of conditions mentioned above. Some of these sources were also included in our spectroscopic survey in the latest years and, as such, they are also considered in the following analysis. 
Table 1. Log of the spectroscopic observations.

\begin{tabular}{|c|c|c|c|c|c|c|}
\hline Run & Telescope & Instrumentation & $\overline{\overline{\text { Dates }}}$ & $\begin{array}{c}\text { Dispersion } \\
(\AA / \text { pix })\end{array}$ & $\begin{array}{c}\text { Spectral range } \\
(\AA)\end{array}$ & $\begin{array}{l}\text { Number of } \\
\text { obs. objects }\end{array}$ \\
\hline$\# 1$ & 3.6 m ESO La Silla & EFOSC1 & 19-21 March 1988 & 3.79 & $3406-6975$ & 12 \\
\hline \#2 & 1.5 m ESO La Silla & Boller \& Chivens & 2-4 January 1989 & 2.47 & $4272-6812$ & 24 \\
\hline \#3 & 1.5 m ESO La Silla & Boller \& Chivens & 23-25 February 1990 & 2.80 & $4046-6920$ & 41 \\
\hline$\# 4$ & $1.5 \mathrm{~m}$ ESO La Silla & Boller \& Chivens & 25-29 June 1990 & 3.47 & $4050-6925$ & 34 \\
\hline \#5 & 2.5 m INT La Palma & IDS & 23-25 Маy 1991 & 2.81 & $3570-5226 / 5538-7240$ & 4 \\
\hline \#6 & $2.2 \mathrm{~m}$ Calar Alto & Boller \& Chivens & November 1991 & 2.50 & $3830-6736$ & 27 \\
\hline \#7 & 1.5 m ESO La Silla & Boller \& Chivens & 19-25 August 1992 & 2.83 & $3590-9425$ & 34 \\
\hline \#8 & 1.5 m ESO La Silla & Boller \& Chivens & 10-13 March 1993 & 3.74 & $3321-11015$ & 32 \\
\hline \#9 & $2.5 \mathrm{~m}$ INT La Palma & IDS & 1-4 July 1993 & 1.48 & $5376-7269$ & 8 \\
\hline \#10 & 1.5 m ESO La Silla & Boller \& Chivens & 13-17 March 1994 & 3.74 & $3285-10980$ & 38 \\
\hline$\# 11$ & 2.5 m INT La Palma & IDS & 18-24 August 1994 & 1.57 & $3779-5401 / 5570-7195$ & 32 \\
\hline \#12 & 1.5 m ESO La Silla & Boller \& Chivens & 11-15 February 1995 & 3.79 & $3532-11190$ & 21 \\
\hline \#13 & 2.5 m INT La Palma & IDS & 13-16 June 1995 & 1.57 & $3700-5455 / 5488-7255$ & 9 \\
\hline \#14 & $2.5 \mathrm{~m}$ INT La Palma & IDS & 9-10 April 2001 & 3.30 & $3702-7401$ & 4 \\
\hline \#15 & 3.6 m ESO La Silla & EFOSC2 & 23-25 June 2003 & 5.30 & $3600-9200$ & 45 \\
\hline
\end{tabular}

The sample presented in this paper is thus comprised by a subset of sources taken from the two GLMP samples described above for which no identification was available (or was poorly established) at the time when these two catalogues were created, and for which optical spectroscopy was obtained.

A total of 253 different IRAS fields were searched, resulting in the successful identification of 205 optical counterparts.

With these selection criteria, our sample of post-AGB stars with optical counterparts is expected to be essentially complete, limited only by the IRAS sensitivity. Only a few C-rich postAGB stars showing prominent solid state features attributed to $\mathrm{SiC}$ at $11.3 \mu \mathrm{m}$, and thus located in region VII of the IRAS colour-colour diagram, as defined by van der Veen \& Habing (1988), may have escaped our identification. In addition, some post-AGB stars in the vicinity of the Galactic Centre could have also been missed due to the IRAS confusion in that region.

\section{Observations and data reduction}

The spectroscopic observations were conducted during several runs spanning 15 years from March 1988 to June 2003. The observations from the Southern Hemisphere were carried out in most cases at the European Southern Observatory (ESO, La Silla, Chile) with the $1.5 \mathrm{~m}$ ESO telescope, equipped with a Boller \& Chivens spectrograph. The first and last runs of observations were carried out at the $3.6 \mathrm{~m}$ ESO telescope, located at the same site, using the ESO Faint Object Spectrograph and Camera EFOSC1 in the first run and EFOSC2 in the last one. The observations from the Northern Hemisphere were carried out at the $2.5 \mathrm{~m}$ Isaac Newton Telescope at the Observatorio del Roque de los Muchachos (La Palma, Spain), using the IDS spectrograph, and at the $2.2 \mathrm{~m}$ telescope at the Observatorio HispanoAlemán (Calar Alto, Spain), also equipped with a Boller \& Chivens spectrograph.

The full log of the spectroscopic observations is shown in Table 1, where we list the telescopes and dates of the observations, together with the instrumentation used in each run, as well as the spectral resolution and the spectral range covered in each case. Exposure times were in the range from 10 to $60 \mathrm{~min}$ depending on the brightness of the source observed, with mean exposure times of 20-30 min.

The optical spectra were reduced using IRAF, following standard techniques. After subtracting the bias, the twodimensional CCD spectra were divided by the normalised flat-field of the corresponding night. Then, the cosmic ray events were identified and removed.

The sky contribution was determined by averaging two narrow regions of the CCD located at both sides of the object and then the mean sky was subtracted. For the wavelength calibration, several $\mathrm{Cu}-\mathrm{Ne}$ or $\mathrm{Cu}-\mathrm{Ar}$ lamp exposures were taken.

Several photometric standards were observed during each observing night and were used for flux calibration. The spectra of the target stars were flux calibrated and corrected for atmospheric extinction using these standards. We estimate typical errors in the absolute fluxes of $\sim 20 \%$. These errors depend mainly on the stability of the seeing conditions for each night of observation.

In parallel with these spectroscopic observations, we also carried out a near infrared photometric survey (Manchado et al. 1989a; García-Lario et al. 1990, 1997). The data obtained in the near infrared helped in many cases to identify the right optical counterpart of the IRAS source, or to confirm the lack of it, in the case of the most obscured sources.

The counterpart identification strategy was the following: in many cases, before the observations took place, a single optical counterpart, close to the IRAS position, was identified in the Digitized Sky Survey (DSS) plates as the most plausible one. When no other information was available, this was the source observed at the telescope.

Sometimes, however, several faint sources with similar brightness were found located within the elliptical IRAS error box. In this case, we usually tried to observe the field in the near-infrared, where we identified the tentative counterpart on the basis of its peculiar near infrared colours (usually the brightest source or the most heavily reddened in the near infrared) first. If no near infrared information was available at the time of the spectroscopic observations, we usually took spectra of all the optical sources located in the field within the elliptical IRAS error box, starting from the closest one to the nominal IRAS position and/or the redder one.

In some cases the identification of the optical counterpart was clear (e.g., when a PN or a source displaying $\mathrm{H} \alpha$ emission was found). Sometimes, however, no special features were detected in the spectra of any of the stars tried, and it was more difficult to determine which of them (if any) was the star physically associated with the IRAS source. It might also be the case that none of the observed stars is the optical counterpart of the IRAS source, since stars on their way to becoming new PNe 
Table 2. Log of the direct imaging observations.

\begin{tabular}{lccc}
\hline \hline Run & Telescope & Instrumentation & Dates \\
\hline \#a & 3.6 m ESO (La Silla) & EFOSC1 & June 1990 \\
\#b & JKT (La Palma) & CCD & July 1990 \\
\#c & 0.90 m Dutch (La Palma) & CCD & March 1994 \\
\#d & 2.5 m NOT (La Palma) & ALFOSC & June 2000 \\
$\#$ e & 2.5 m NOT (La Palma) & ALFOSC & May 2001 \\
$\# f$ & 3.6 m ESO (La Silla) & EFOSC2 & June 2003 \\
\hline
\end{tabular}

can become so heavily obscured by the material expelled during the previous AGB phase that they may not be detectable in the optical range. We will further discuss these uncertainties in Sect. 4.1.

The full list of the 253 IRAS fields observed is shown in Table 3 (only available in electronic format), where we also give: the entry number of the object in the GLMP catalogue, the IRAS name, the improved astrometric coordinates taken from the 2MASS Point Source Catalogue (Cutri et al. 2003) for which the estimated errors are of the order of $\sim 0.2^{\prime \prime}$ corresponding to the source identified as the right counterpart, and the far infrared IRAS colours [12]-[25] and [25]-[60], defined in the classical way as:

$$
\begin{aligned}
& {[12]-[25]=-2.5 \log \frac{F_{12 \mu \mathrm{m}}}{F_{25 \mu \mathrm{m}}}} \\
& {[25]-[60]=-2.5 \log \frac{F_{25 \mu \mathrm{m}}}{F_{60 \mu \mathrm{m}}} .}
\end{aligned}
$$

In the last columns of this table, we also provide the classification assigned to each source and, in the case of the non-evolved objects, the spectral class or type, and we identify the spectroscopic observing run when every source was observed, according to the code given in Table 1.

In addition to the spectroscopic observations, CCD images of selected fields were also obtained through various standard broad and narrow filters covering the whole optical range and using several telescopes listed in Table 2. These images were bias and flat-field corrected and cleaned for cosmic rays using standard IRAF routines. No flux calibration was performed in this case.

In Appendices A-F (only in electronic format) we show the spectrum and the finding chart for all the targets in the survey for which an optical counterpart was identified and a spectrum was taken. In most cases the finding charts are taken from the DSS. However, when extended emission was detected in our images, we used them for the atlas.

We prefer showing the images obtained through red filters (usually the $R$-band) if available, because in most cases the optical counterparts of the IRAS sources in our sample are expected to be brighter at longer wavelengths since on many occasions they are strongly reddened by their circumstellar envelopes. If convenient, we have also used our $\mathrm{H} \alpha$ images, in some cases, to better illustrate the morphology of those sources showing extended ionised emission.

\section{Results}

\subsection{Classification of the sources in the sample}

To classify the objects in the sample as a function of their nature and evolutionary stage, we have studied their optical spectra and the complementary information coming from the near infrared measurements made by García-Lario et al. (1997) and from other sources in the literature. The assigned classifications are shown in Table 3.

The determination of the evolutionary stage of an object can lead to some uncertainties, especially when trying to discriminate between post-AGB stars and young stellar objects. If the source is located within the boundaries or close to a known starforming region, we have assumed it is most probably young. The luminosity obtained in the spectral classification can also give us some hints about its evolutionary stage, since the young stars are expected to show spectra corresponding to a low luminosity class (usually V), while post-AGB stars show extended atmospheres and low gravities usually corresponding to the high luminosity classes I and III.

To take these uncertainties into account, we have introduced a two-letter code in Tables 4-6. The letters rang from A to D and rate our confidence in the data presented, with A representing the maximum reliability. The first letter marks our confidence in the correct identification of the optical counterpart, and the second letter indicates how confident we are in the evolutionary classification assigned.

The adopted selection criteria turned out to be very efficient in the detection of evolved stars. Up to 209 sources, representing $81 \%$ of the stars in the sample, were found to be evolved stars. Among them, the majority of these sources are considered to be post-AGB stars: 103 with an optical counterpart (see Table 4), plus possibly 49 more heavily obscured ones, for which we did not find any optical counterpart during our observations, although most of which are believed to be obscured post-AGB stars. They are labeled as "No counterpart" in Table 3. We have also found 21 "transition sources" (see Table 5).

Only 38 of the sources observed in the sample ( 17\%) were identified as young stellar objects and 2 other ones as galaxies (less than 1\%). Their identifications and the spectral type of the young objects are shown in Table 3.

\subsection{Classification of the optical spectra}

\subsubsection{Spectral types in the MK system}

The classification of the optical spectra in the MK system was performed taking several spectral libraries as references (Silva \& Cornell 1992; Jacoby et al. 1984; Pickles 1998), and it was applied to all the observed sources showing a stellar continuum. By default, we always assumed a luminosity class I in our first try for the sources suspected to be post-AGB stars. To perform this classification, we first normalised both the spectral templates taken from these catalogues and our target spectra. This way we concentrate our attention on the spectral lines, rather than on the stellar continuum, which may appear extremely reddened in many stars of our sample.

We used Silva \& Cornell's templates for the spectra with the lowest signal-to-noise ratios and for the young stellar objects. The estimated error in this classification is about plus or minus one subclass, which implies a total error of around five subtypes in the determination of the spectral type.

For the sources in our sample classified as evolved objects whose spectra had a good signal-to-noise ratio, we used the other two catalogues (Jacoby et al. 1984; Pickles 1998) to derive a more accurate spectral classification, as they provide one spectrum for each subtype and for a wide range of spectral types for stars with luminosity class I. We estimate that the stars classified using these catalogues are affected by errors that are always of the order of less than two spectral subtypes. 
We have had difficulties classifying with accuracy the stars with the earliest spectral types showing the Balmer lines in emission. In many cases these lines show emission over absorption, making it impossible to use their strength for classification purposes.

In Table 4 we list all the sources in the sample classified as post-AGB stars together with their associated spectral classification, and in Table 5 we show the classification of the transition sources ${ }^{1}$.

The spectral type in these two tables is given, as usual, by the letter and number corresponding to the MK classification system. Those spectra for which Silva's rough classification was used maintain the nomenclature followed in that catalogue, i.e, one letter followed by two numbers indicating the range of subtypes. The letter "e" appended to the spectral type indicates that the object shows emission lines. When the spectrum is dominated by emission lines, and the continuum is too faint to derive its spectral type, it is classified with the code "em".

\subsubsection{Determination of the extinction constant, the excitation classes for PNe, and the WR type of the central stars}

To determine the extinction corrected line intensities of the $\mathrm{PNe}$, we calculated the extinction coefficient $c$, using the observed Balmer line decrement (see results in Table 6). Then we dereddened the observed spectra using Whitford's extinction law (Whitford 1958), and we used the dereddened lines to calculate the excitation classes.

The excitation classes were determined using both Morgan (1984) and Dopita \& Meatheringham (1990) criteria to provide a more reliable classification. Morgan (1984) based this classification on the value of the ratios [O III] $(\lambda 4959 \AA) / \mathrm{H} \beta$, $\mathrm{H} \beta / \mathrm{HeII}(\lambda 4686 \AA)$, and $\mathrm{H} \beta /[\mathrm{NeIII}](\lambda 3869 \AA)$. It establishes 12 different classes, ranging from low (class 0 ) to high (class 10) excitation. The classification proposed by Dopita \& Meatheringham (1990) is a continuous one, based on the ratios [O III] $(\lambda 4959 \AA) / \mathrm{H} \beta$ and HeII $(\lambda 4686 \AA) / \mathrm{H} \beta$.

We grouped together all possible excitation classes in only three main subgroups: low, medium, and high excitation (see Table 6).

Note that some PNe were impossible to classify using these criteria, as they appeared so extinguished in the blue region that they did not show several of the lines needed for the classification.

In the case of the PNe with H-poor envelopes (i.e., with WR-type central stars), we used the criteria defined by Acker \& Neiner (2003) which is, in turn, also based on the classification previously defined by Crowther et al. (1998). They identify [WO] subtypes ranging from [W01] to [W04] and [WC] subtypes ranging from [WC4] to [WC11]. This classification is based on the dereddened strength of several $\mathrm{O}$ and $\mathrm{C}$ lines with respect to the continuum, and implies a decrease in temperature from $100000 \mathrm{~K}$ for [WO1-3] stars to 20000 for [WC10].

The final classification derived for the PNe found in our spectroscopic survey and additional information on the morphology of individual sources is shown in Table 6.

1 The rough spectral types assigned to the young stellar objects identified in our survey are shown in Table 3. Note that for these sources we did not try to make any luminosity class determination.

\section{Discussion}

In the following we will concentrate our discussion on the subsample of evolved stars, e.g., the sources classified either as optically bright post-AGB stars, transition sources or PNe, plus the subsample of heavily obscured sources also tentatively classified as post-AGB stars.

\subsection{Galactic latitude distribution}

To study the evolutionary connection between the various types of evolved stars identified in this work (post-AGB stars with and without optical counterpart, transition sources, and PNe) we can compare their relative galactic latitude distribution. For a given class, this is expected to be an indicator of the average mass of the progenitor stars. The youngest, and thus more massive, stars are expected to appear more concentrated towards the galactic plane, while an older population of stars, with lower masses, should show a higher dispersion.

For this purpose, in Fig. 1, we have plotted the galactic latitude distribution of the optically bright post-AGB stars and transition sources, PNe, and obscured post-AGB stars identified in our sample.

To test if the apparent differences among the distributions shown in Fig. 1 are statistically significant, we have used the FSnedecor test (or F-variance test). This test determines whether two samples are drawn from populations with the same variances.

The result of this study shows that there are no statistically significant differences between the galactic distribution of optically bright post-AGB stars (including the transition sources) and the galactic distribution of PNe with a $99 \%$ confidence. This can be interpreted as the confirmation that an evolutionary link exists between the two populations.

However, the galactic distribution of post-AGB stars with no optical counterparts seems to be different from both the postAGB stars with optical counterparts and the PNe. The narrow galactic latitude distribution of the obscured post-AGB stars suggests that they represent a population of more massive progenitors. These stars may be evolving very rapidly to the PN stage and have very recently left the AGB phase, as they still preserve the optically thick envelopes formed in the mass-losing phase. In contrast, the post-AGB stars showing a bright optical counterpart may represent a less massive population of stars that would evolve more slowly. Alternatively, they may never develop optically thick envelopes, evolving all the way from the end of the AGB to the PN stage as optically bright stars.

\subsection{Spectral type distribution of post-AGB stars}

We have found post-AGB stars belonging to all spectral types from $M$ to $B$, shown in Fig. 2, in what can be interpreted as an evolutionary sequence towards higher effective temperatures on their way to becoming PNe. If this interpretation is correct, the distribution of spectral types should be a good indicator of the time spent by these stars in each range of temperatures. The same analysis could be applied to high mass stars, but in this case, part of the evolution would take place while the central star is still heavily obscured by the circumstellar envelope, and by the time it could be observed in the optical, it would have already evolved significantly towards hotter temperatures.

The models developed by (van Hoof et al. 1997, VH97, hereafter) predict the relative number of stars that we should expect to find within each spectral type. 

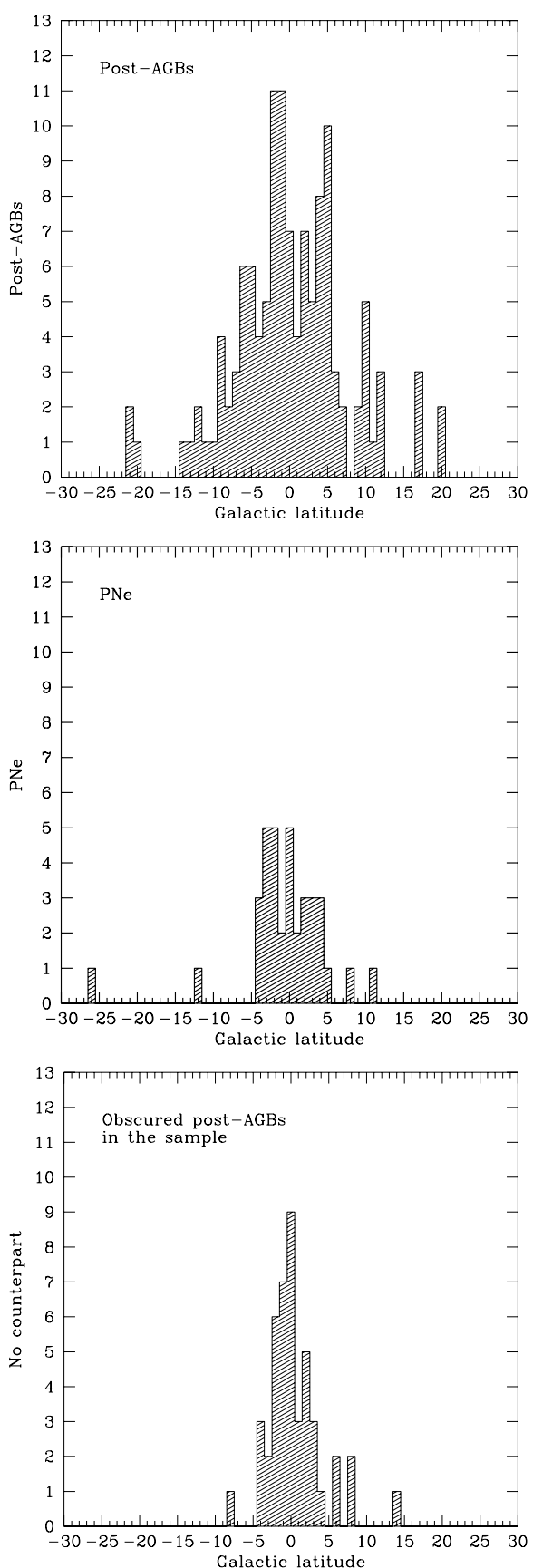

Fig. 1. Galactic latitude distribution of optically bright post-AGB stars, $\mathrm{PNe}$, and obscured post-AGB stars identified in the sample.

To test if the distribution of spectral types observed (see Fig. 2) is similar to any of the models produced by VH97, we have applied the Pearson's Chi-square test. This test verifies if the shape of the distribution of a given variable fits a theoretical distribution with known parameters reasonably well.

The result of this analysis indicates that the distribution of spectral types observed in our sample of post-AGB stars and transition objects, excluding the M- and K-spectral types, is not compatible with any of the three theoretical models used by VH97 with a $95 \%$ confidence level.

We suggest that the incompatibility can be due to the fact that our sample is a combination of objects with different core masses, as is to be expected from the selection criteria chosen, which do not favour any particular core mass range.

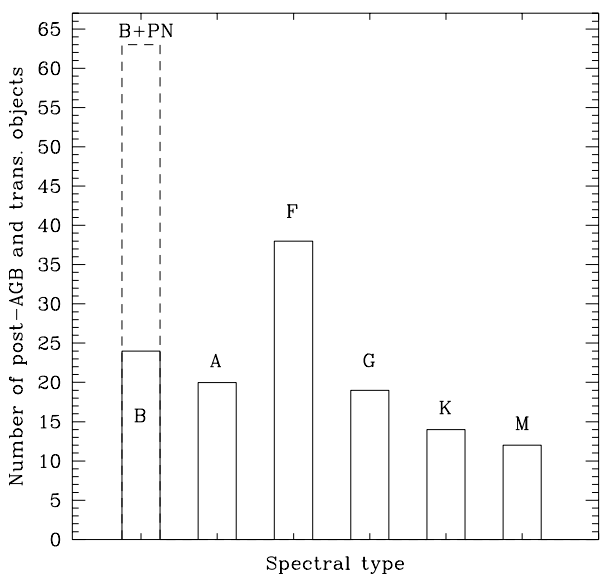

Fig. 2. Relative distribution of spectral types for the post-AGB stars in our sample.

\subsection{Distribution in the IRAS colour-colour diagram}

\subsubsection{Overall distribution}

In Fig. 3 in the IRAS colour-colour diagram, we show the distribution of all the sources included in our spectroscopic survey, where special symbols are used to distinguish between the different types of objects: PNe, optically bright post-AGB stars, galaxies, young stellar objects, and objects for which no counterpart was found (transition sources have again been included together with the post-AGB stars).

The boxes with dashed boundary lines indicate areas mostly populated by variable OH/IR stars (box $b$, dotted line), T-Tauri and Herbig Ae/Be stars (box $c$, short dash), and compact HII regions (box $e$, long dash) (see García-Lario et al. 1997, and references therein). In Fig. 3 we have maintained the labels used in that paper.

The two solid straight lines (vertical and horizontal) show the limits of the area defined by our selection criteria (see Sect. 2). The solid curve has been modeled by Bedijn (1987), and shows the location of O-rich stars as they evolve along the AGB from M-type Miras to variable $\mathrm{OH} / \mathrm{IR}$ stars.

We can see that PNe and post-AGB stars spread almost regularly over the diagram. However, it is important to remark that there is a high probability ( $>95 \%$ ) of finding a post-AGB or a $\mathrm{PN}$ in the region that does not overlap with regions $b, c$, or $e$.

Almost all of young sources (marked with open triangles) are located, as expected, either in box $c$ (T-Tauri, Herbig Ae/Be, and Vega-like stars) or $e$ (usually populated by protostars and ultra-compact $\mathrm{H}$ II regions).

We have also plotted the objects for which we did not find any optical counterpart as small asterisks. A few of them are located in box $e$, suggesting that they could probably be heavily obscured young stellar objects, surrounded by thick protoplanetary discs or by their parent molecular cloud in which they are embedded. The rest of the sources with no counterpart are more or less clustered around a value of [25]-[60] -0.2 and/or relatively close to box $b$, and these are the ones that have been classified as heavily obscured post-AGB stars in this paper.

\subsubsection{Post-AGB stars and transition objects}

In Fig. 4 we show more in detail the distribution of post-AGB stars and "transition sources" in the IRAS two-colour diagram as a function of the spectral type. Post-AGB stars with different spectral types are plotted with different colours, and a different 


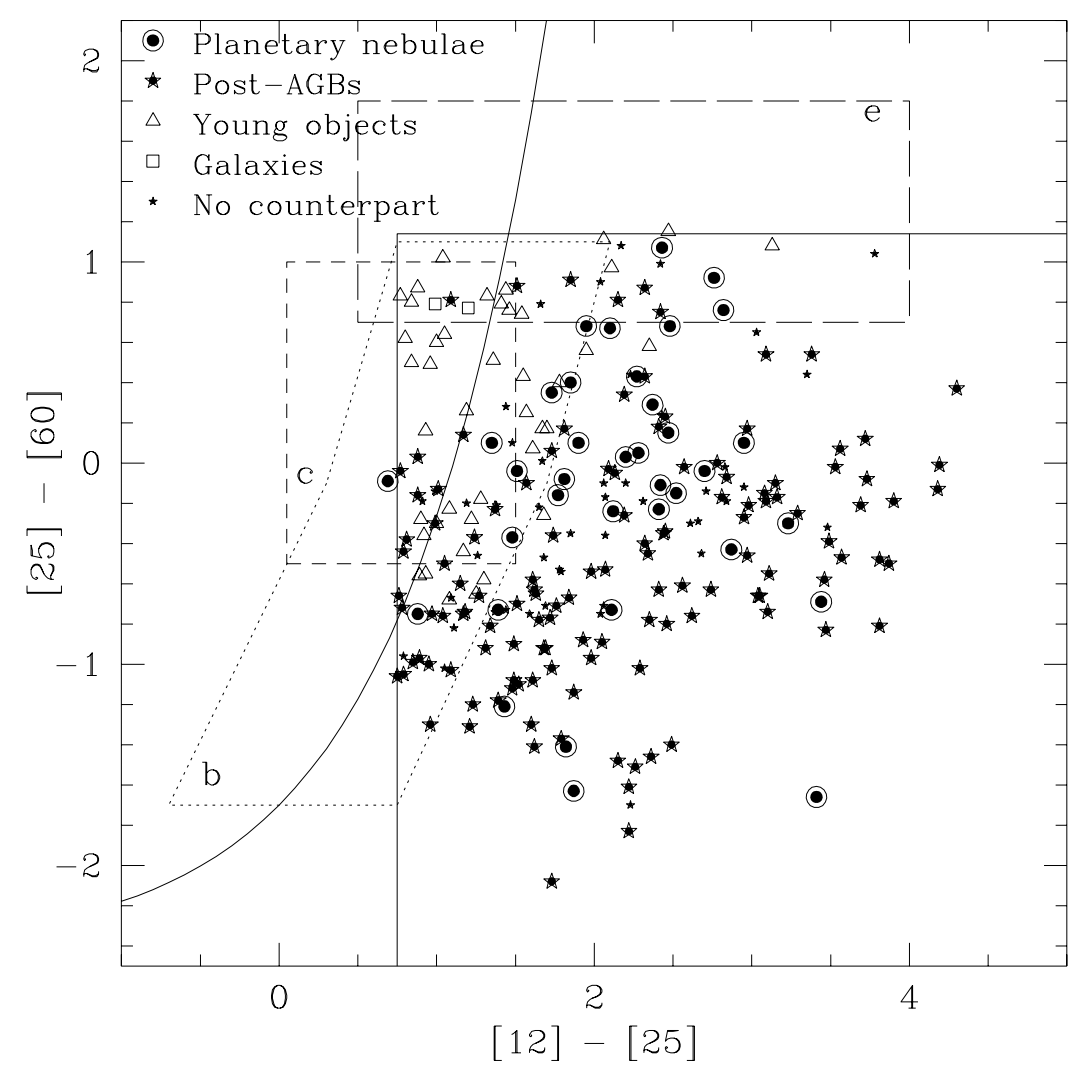

Fig. 3. IRAS colour-colour diagram showing the location of all the objects included in our spectroscopic survey.

symbol is used to plot the transition objects. Objects showing $\mathrm{H} \alpha$ emission are surrounded with a circle. Sources with emission, but for which we could not determine the spectral type because of their faint continuum, are plotted as open circles.

It is already known that there is not a one-to-one correspondence between the position of a post-AGB star in the IRAS twocolour diagram and its evolutionary stage (VH97), but this is the first time that a large sample of spectroscopically classified postAGB stars show this evidence.

Neither the C-rich models nor the O-rich models predict an unambiguous relationship between the evolutionary stage in the post-AGB phase and the position in the IRAS two colour diagram. Perhaps models for different core masses and precise temporal marks could be used to create statistics and check whether there is an adequate correspondance between the position of our objects and their evolutionary status.

\section{Conclusions}

We have presented the results of an extensive spectroscopic survey carried out on a sample of 253 IRAS sources showing infrared colour characteristics of PNe. The selection criteria used turned out to be very efficient in discovering new post-AGB stars and transition sources evolving from the AGB to the PN stage, resulting in the identification of 152 post-AGB stars (49 of them without an optical counterpart), 21 transition sources, and $36 \mathrm{PNe}$. This constitutes the largest catalogue of post-AGB stars built so far.

The results from the survey are presented in an atlas format, including the spectroscopic data and finding charts. Improved astrometric information and spectral classification is also provided.
Our spectroscopic study has substantially increased the knowledge of the optical properties of galactic post-AGB stars, as can be seen in Table 4; only 35\% of our 103 objects had their spectral types identified in the SIMBAD database.

An analysis of the galactic latitude distribution of the sources in the sample shows that the new population of obscured postAGB stars identified in our spectroscopic survey must in general have higher progenitor masses than the population of post-AGB stars with optical counterparts for which spectroscopic data is presented in the atlas. The relatively large number of post-AGB stars showing optically thick envelopes suggests that many stars may evolve during a significant part of their post-AGB evolution hidden from detection in the optical.

The similar galactic latitude distribution shown by the PNe included in our sample and the population of post-AGB stars with optical counterparts suggests that many of these post-AGB stars will develop observable PNe, as they seem to belong to the same galactic population. Unfortunately, we do not know whether the small sample of PNe here studied is representative of the more general population of galactic PNe.

The spectral type distribution of the post-AGB stars observed is not compatible with any of the theoretical models developed by VH97 to predict the distribution expected for stars with several specific core masses. We suggest that this is due to our sample being a combination of sources with a wide variety of core masses.

The IRAS two-colour diagram has proved to be very useful in finding new post-AGB stars. The distribution of the post-AGB stars and the PNe in the diagram is similar, and suggests a direct evolutionary connection. We have also confirmed that the precise location of a given post-AGB star or a PNe in this diagram by itself cannot be directly interpreted as an indication of their evolutionary stage, as was suggested by previous theoretical works. 


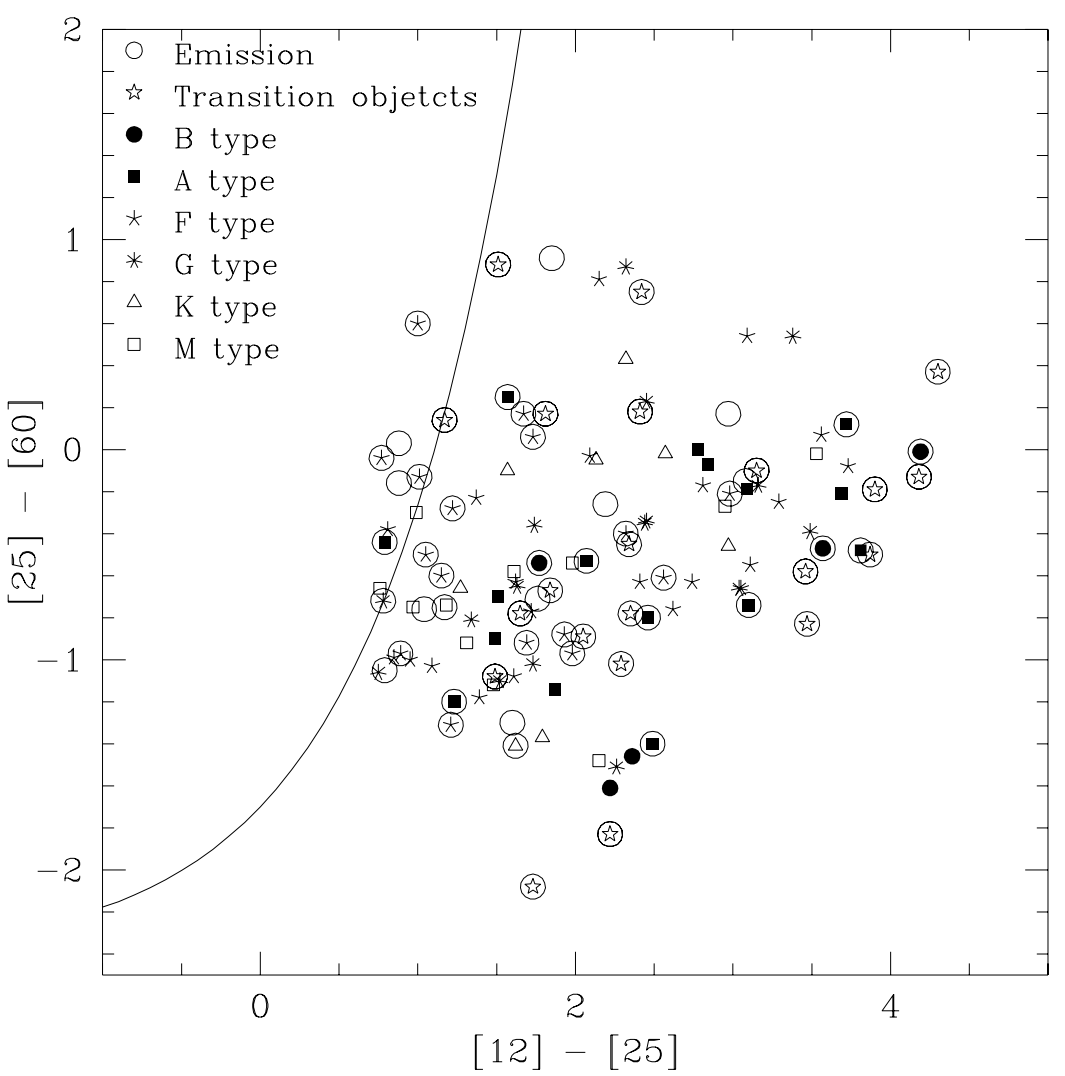

Fig. 4. IRAS colour-colour diagram showing the location of the post-AGB stars and transition objects in the survey.

Acknowledgements. IRAF is distributed by the National Optical Astronomy Observatories, which are operated by the Association of Universities for Research in Astronomy, Inc., under cooperative agreement with the National Science Foundation.

This research was funded through grant AYA-2003-09499 from the Spanish Ministerio de Ciencia y Tecnología. A.M. acknowledges support from grant AYA2004-03136 funded by the Spanish Ministerio de Educacion y Ciencia. It made extensive use of the Simbad database, operated at CDS, Strasbourg (France), as well as of the Space Telescope Science Institute Digitized Sky Survey (DSS). The STScI DSS is based on photographic data obtained using the UK Schmidt Telescope and produced at the STScI under US Government grant NAG W-2166. Some images presented in the Appendices were obtained at the $2.5 \mathrm{~m}$ Nordic Optical Telescope, operated jointly by Denmark, Finland, Iceland, Norway, and Sweden, also at the Spanish Observatorio del Roque de los Muchachos of the Instituto de Astrofísica de Canarias.

\section{References}

Acker, A., \& Neiner, C. 2003, A\&A, 403, 659

Acker, A., Marcout, J., Ochsenbein, F., Stenholm, B., \& Tylenda, R. 1992, Strasbourg - ESO catalogue of galactic planetary nebulae. Part 1; Part 2 (Garching: European Southern Observatory)

Allen, D. A., Hyland, A. R., \& Caswell, J. L. 1980, MNRAS, 192, 505

Bedijn, P. J. 1987, A\&A, 186, 136

Beichman, C. A., Neugebauer, G., Habing, H. J., Clegg, P. E., \& Chester, T. J. 1988, NASA Reference Publication, 1190, 1

Bobrowsky, M., Sahu, K. C., Parthasarathy, M., \& García-Lario, P. 1998, Nature, 392, 469

Crowther, P. A., de Marco, O., \& Barlow, M. J. 1998, MNRAS, 296, 367

Cutri, R. M., Skrutskie, M. F., van Dyk, S., et al. 2003, VizieR Online Data Catalog, 2246, 0

de Gregorio-Monsalvo, I., Gómez, Y., Anglada, G., et al. 2004, ApJ, 601, 921

Dopita, M. A., \& Meatheringham, S. J. 1990, ApJ, 357, 140

Draine, B. T., \& Lee, H. M. 1984, ApJ, 285, 89

García-Lario, P. 1992, Ph.D. Thesis, Universidad de La Laguna, Spain
García-Lario, P., Manchado, A., Suso, S. R., Pottasch, S. R., \& Olling, R. 1990, A\&AS, 82, 497

García Lario, P., Manchado, A., Riera, A., Mampaso, A., \& Pottasch, S. R. 1991, A\&A, 249, 223

García-Lario, P., Manchado, A., Pych, W., \& Pottasch, S. R. 1997, A\&AS, 126, 479

García-Lario, P., Manchado, A., Ulla, A., \& Manteiga, M. 1999a, ApJ, 513, 941

García-Lario, P., Riera, A., \& Manchado, A. 1999b, ApJ, 526, 854

Habing, H. J. 1996, A\&AR, 7, 97

Hu, J. Y., Slijkhuis, S., de Jong, T., \& Jiang, B. W. 1993, A\&AS, 100, 413

Jacoby, G. H., \& van de Steene, G. 2004, A\&A, 419, 563

Jacoby, G. H., Hunter, D. A., \& Christian, C. A. 1984, ApJS, 56, 257

Kwok, S. 1993, ARA\&A, 31, 63

Manchado, A., García-Lario, P., Esteban, C., Mampaso, A., \& Pottasch, S. R. 1989a, A\&A, 214, 139

Manchado, A., García-Lario, P., \& Pottasch, S. R. 1989b, A\&A, 218, 267

Morgan, D. H. 1984, MNRAS, 208, 633

Oudmaijer, R. D. 1996, A\&A, 306, 823

Oudmaijer, R. D., van der Veen, W. E. C. J., Waters, L. B. F. M., et al. 1992, A\&AS, 96, 625

Pickles, A. J. 1998, PASP, 110, 863

Pottasch, S. R., Olling, R., Bignell, C., \& Zijlstra, A. A. 1988, A\&A, 205, 248

Preite-Martinez, A. 1988, A\&AS, 76, 317

Ratag, M. A., Pottasch, S. R., Zijlstra, A. A., \& Menzies, J. 1990, A\&A, 233, 181

Riera, A., García-Lario, P., Manchado, A., Pottasch, S. R., \& Raga, A. C. 1995, A\&A, 302, 137

Scoville, N. Z., \& Kwan, J. 1976, ApJ, 206, 718

Silva, D. R., \& Cornell, M. E. 1992, ApJS, 81, 865

van de Steene, G. C. M., \& Pottasch, S. R. 1993, A\&A, 274, 895

van der Veen, W. E. C. J., \& Habing, H. J. 1988, A\&A, 194, 125

van der Veen, W. E. C. J., Habing, H. J., \& Geballe, T. R. 1989, A\&A, 226, 108 van Hoof, P. A. M., Oudmaijer, R. D., \& Waters, L. B. F. M. 1997, MNRAS, 289,371

van Winckel, H. 2003, ARA\&A, 41, 391

Vassiliadis, E., \& Wood, P. R. 1994, ApJS, 92, 125

Whitford, A. E. 1958, AJ, 63, 201 


\section{Online Material}


O. Suárez et al.: Spectroscopic atlas of post-AGB and PNe, Online Material $p 2$ 


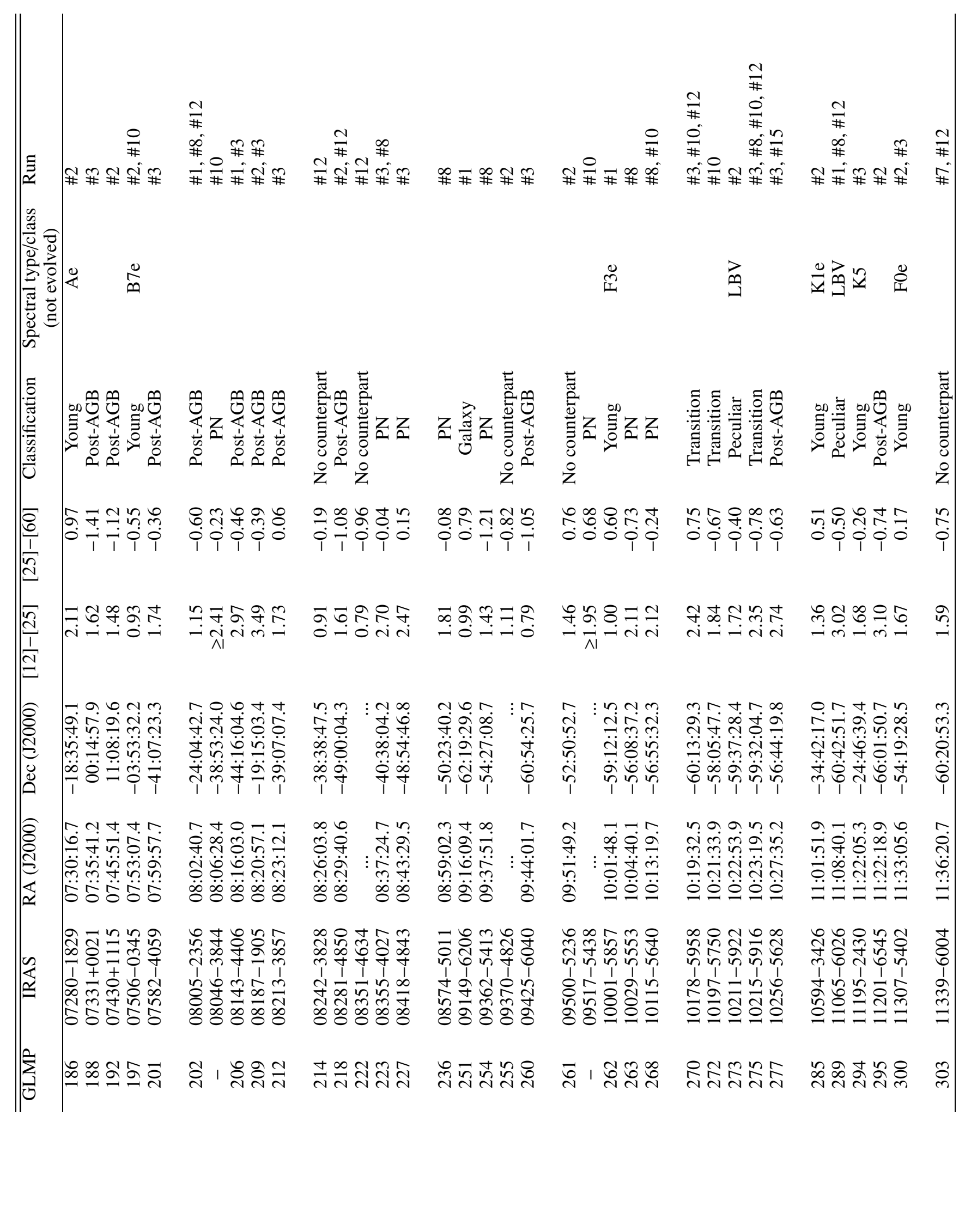




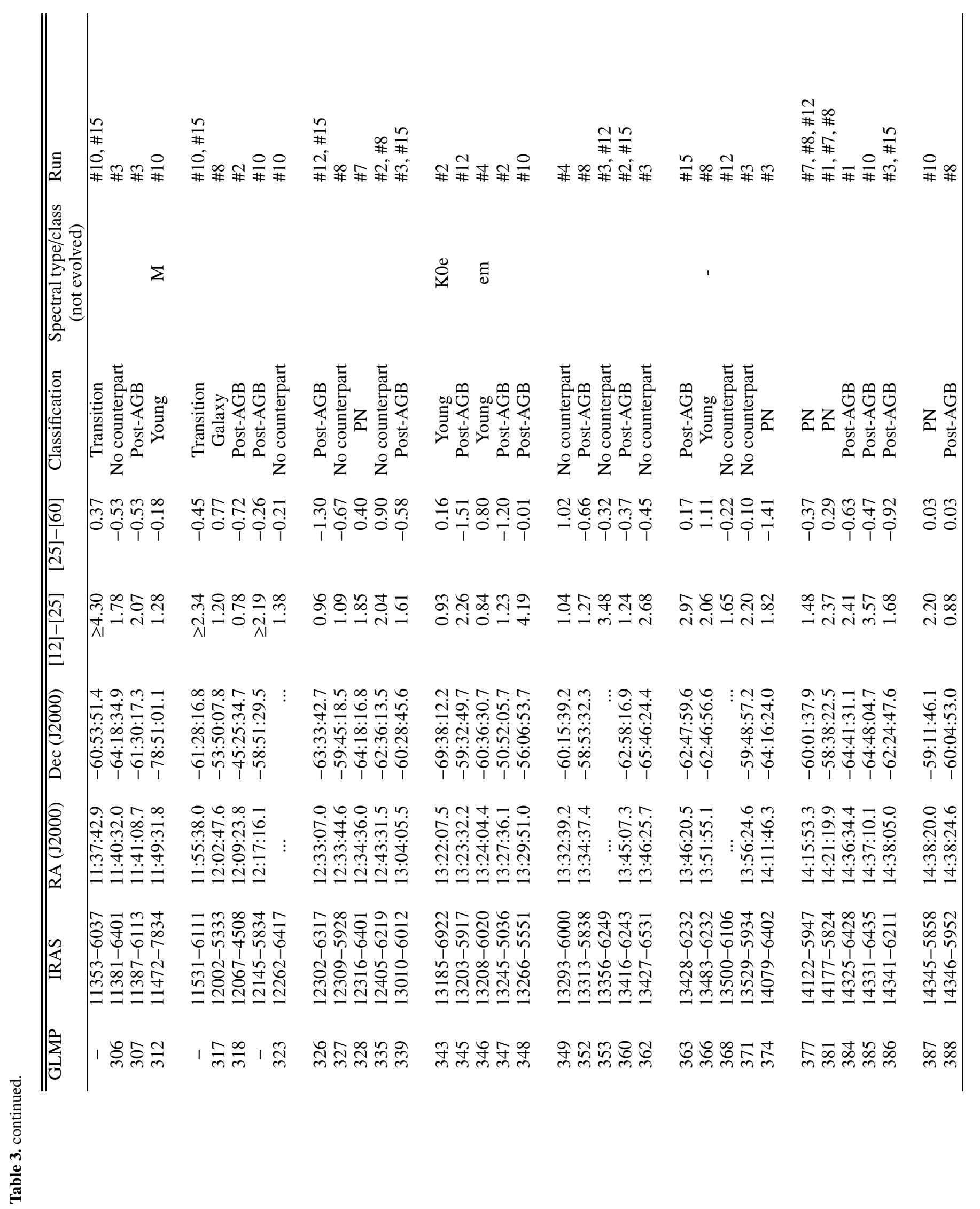




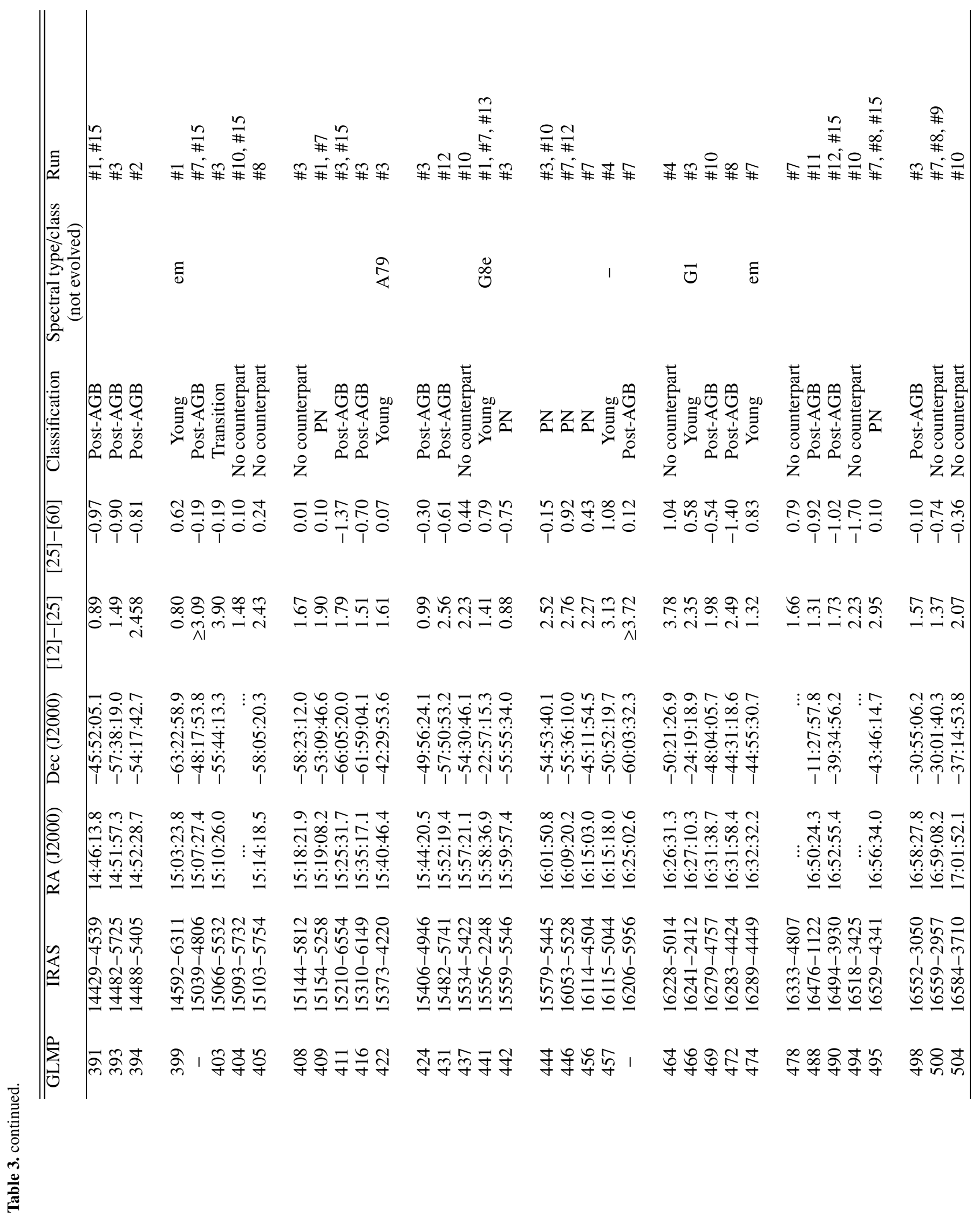




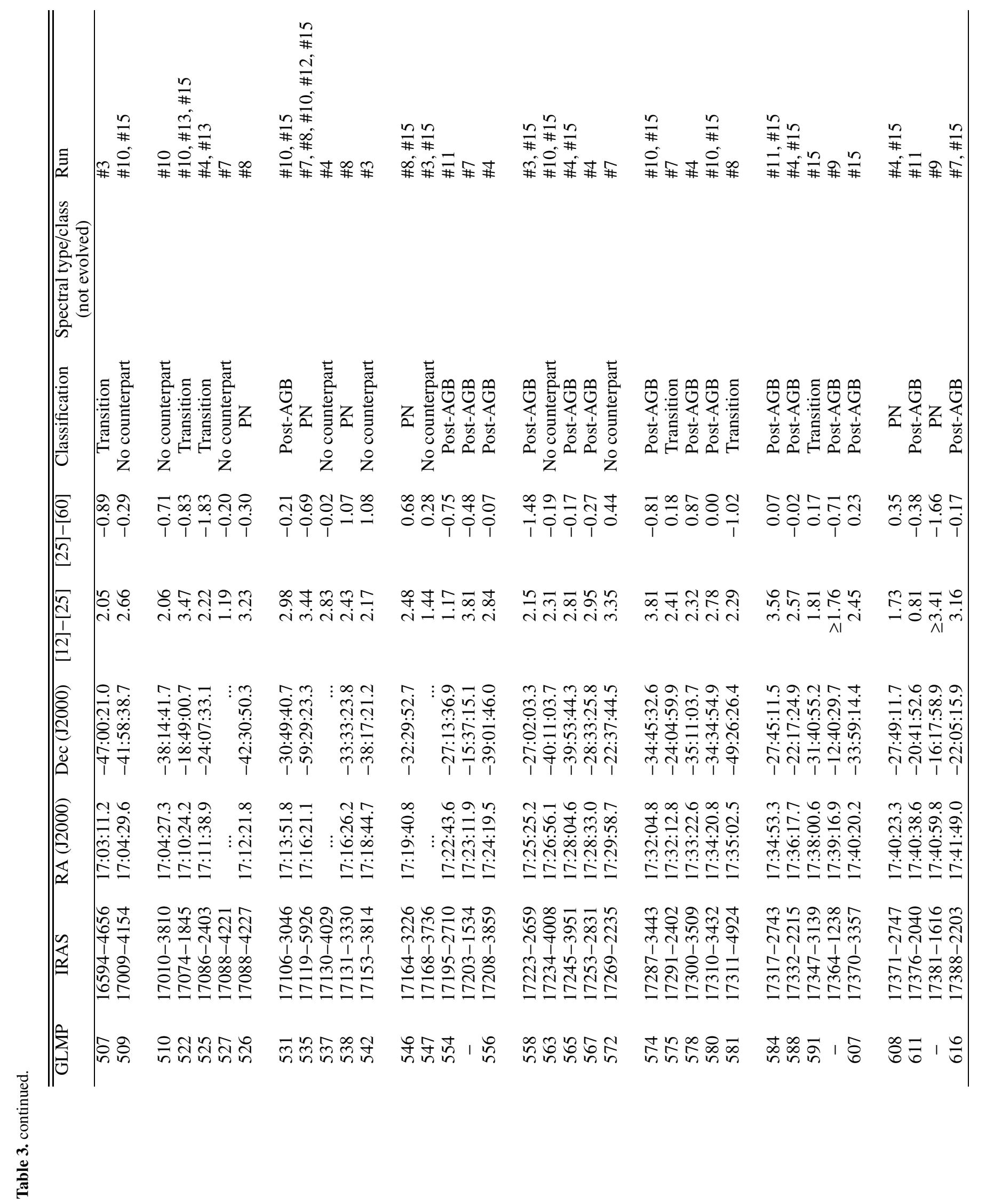


O. Suárez et al.: Spectroscopic atlas of post-AGB and PNe, Online Material $p 7$

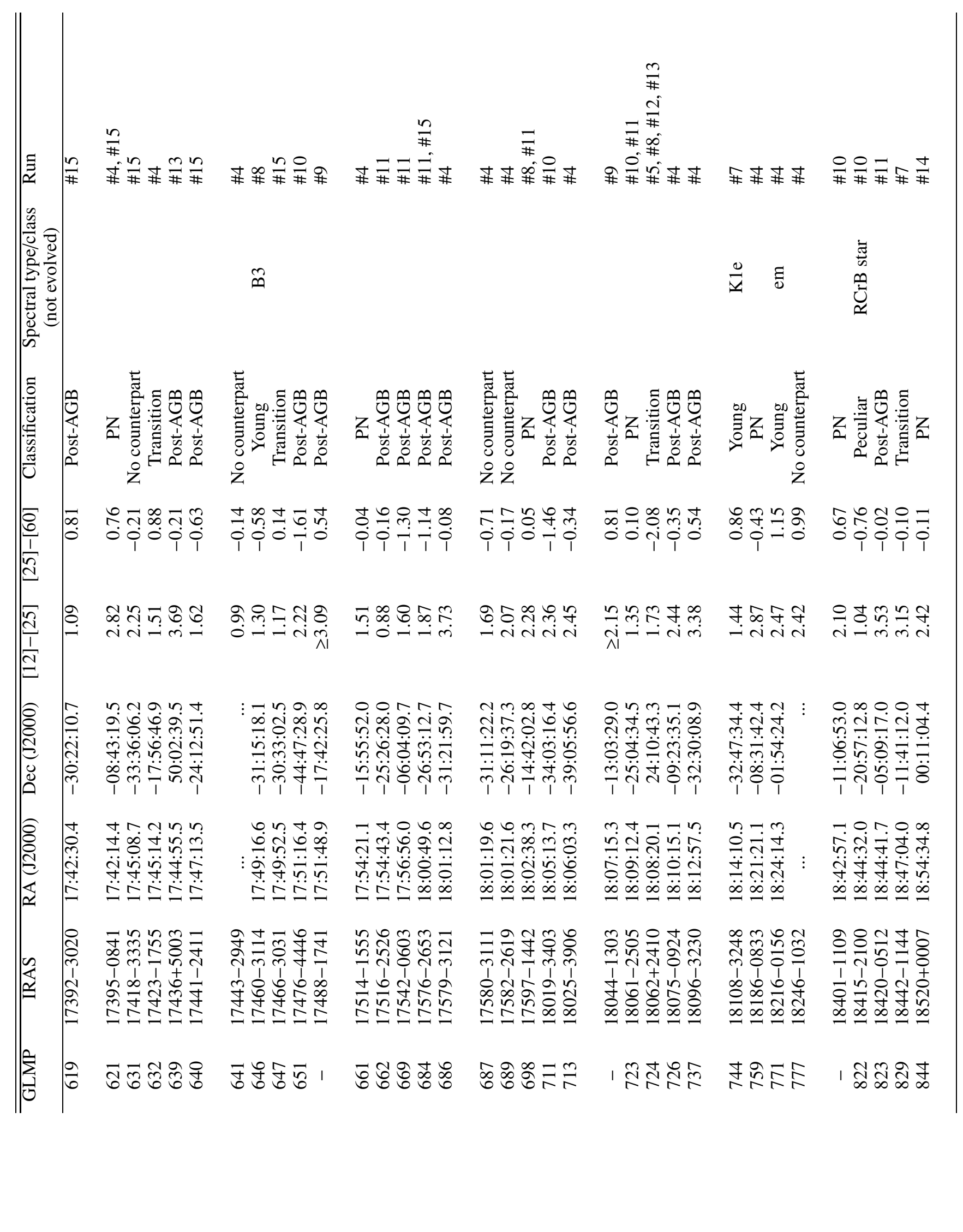


O. Suárez et al.: Spectroscopic atlas of post-AGB and PNe, Online Material $p 8$

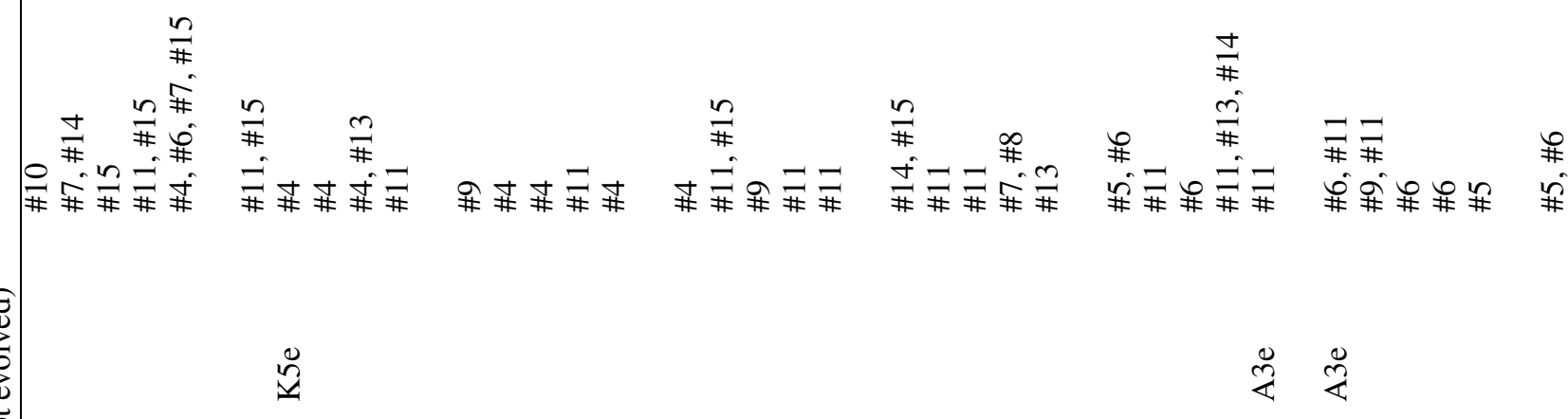

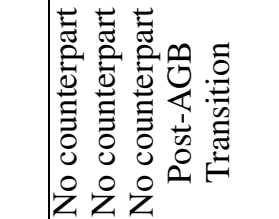

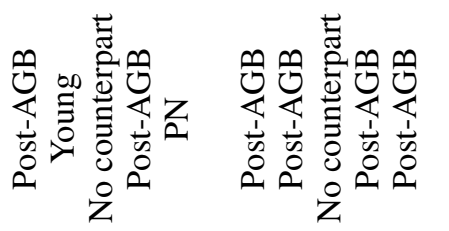

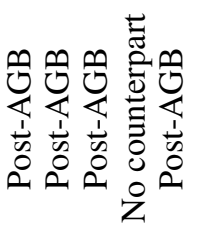

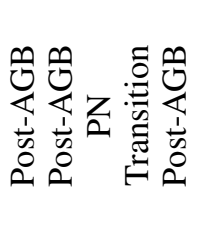
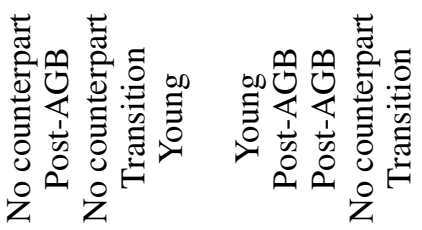

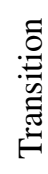

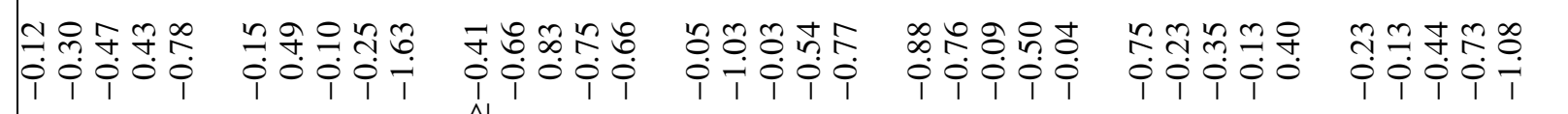

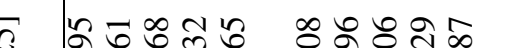

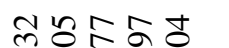

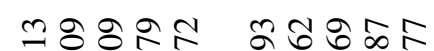

țin $\infty$

串可只寺守

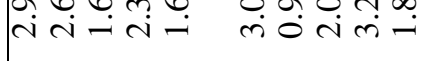

तलं०ठठ

i.

पi ता mio

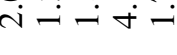

-

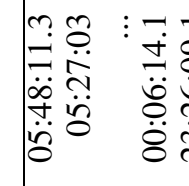

के ت워요

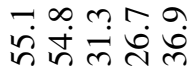

लmim:

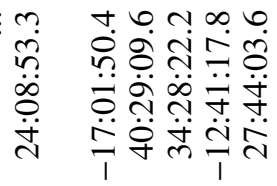

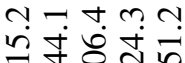

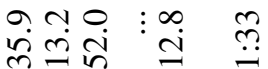

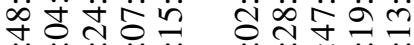

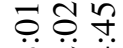

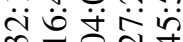

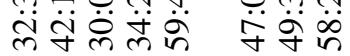

$\ddot{\sim} \dot{\sim}$

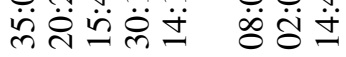

$\stackrel{\infty}{\ddot{\theta}}$
$\stackrel{?}{+}$

ते

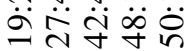

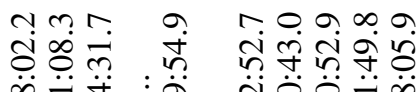

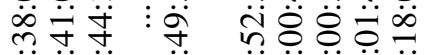

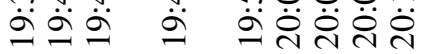

तेंतेंतें

$+0 \infty \quad$

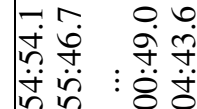

तis

ชั

$\ddot{\vec{\alpha}} \dot{\vec{n}} \dot{\mathrm{d}} \dot{\mathrm{m}}$

$\ddot{\alpha} \dot{\alpha} \dot{g}$

$\dot{a} \dot{a} \ddot{a} \ddot{a}$

$\ddot{\sigma} \ddot{\sigma} \ddot{\sigma} \ddot{\sigma} \ddot{\sigma}$

\section{+}

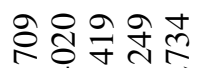

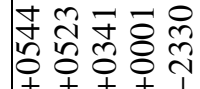

考官家客客

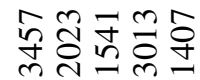

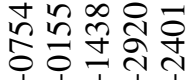

तิ유워ำ

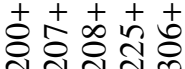

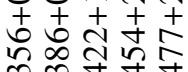

ठे के \&०

$10 \infty-100$

बूरूু

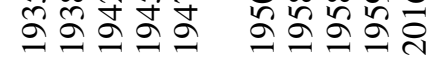

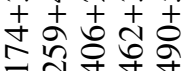

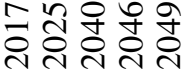

nู

ڤึळ

$\infty \rightarrow \infty$

๙ $\sigma$ ప

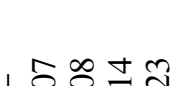

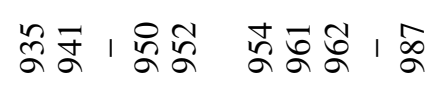

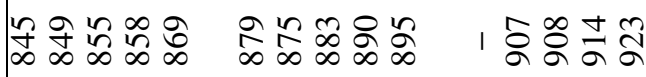

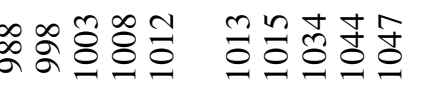


O. Suárez et al.: Spectroscopic atlas of post-AGB and PNe, Online Material p 9

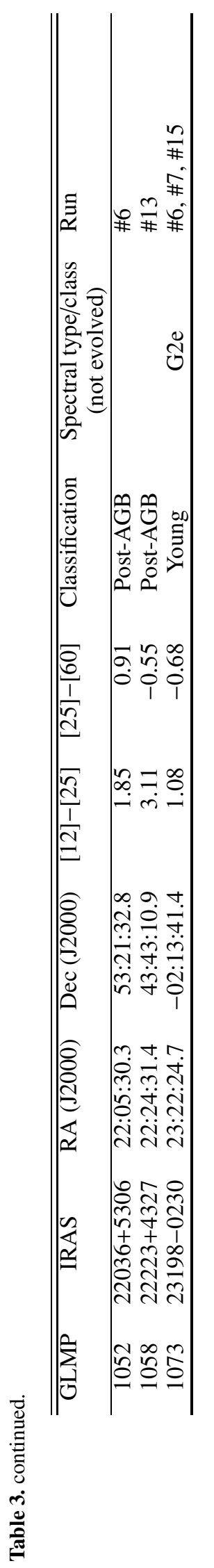


Table 4. Main characteristics derived for the IRAS sources identified as post-AGB stars.

\begin{tabular}{|c|c|c|c|c|}
\hline$\overline{\text { IRAS name }}$ & Other identifications & $\begin{array}{c}\text { Spectral type } \\
\text { our survey }\end{array}$ & $\begin{array}{c}\text { Spectral type } \\
\text { (SIMBAD) }\end{array}$ & $\begin{array}{c}\text { Reliability } \\
\text { code }^{\dagger}\end{array}$ \\
\hline $01005+7910$ & & $\mathrm{Fe}$ & B2Iab:e & $\mathrm{AA}$ \\
\hline $01259+6823$ & & F5Ie & GIab: & AA \\
\hline $02143+5852$ & & F7Ie & A5 & AA \\
\hline $04296+3429$ & & F7I & G0Ia & AA \\
\hline $05089+0459$ & & M3I & M & $\mathrm{AB}$ \\
\hline $05113+1347$ & PM 2-4 & G5I & G8Ia & AA \\
\hline $05341+0852$ & & F5I & F4Iab: & AA \\
\hline $05381+1012$ & & G2I & $\mathrm{G}$ & AA \\
\hline $06530-0213$ & PM 1-24 & G1I & F0Iab: & AA \\
\hline $07134+1005$ & & F7Ie & F5Iab: & AA \\
\hline $07227-1320$ & & M1I & & AA \\
\hline $07253-2001$ & & F2I & & AA \\
\hline $07331+0021$ & & K35I & G5Iab & AA \\
\hline $07430+1115$ & & M2I & G5Ia & AA \\
\hline $07582-4059$ & PM 1-35 & G5I & & AA \\
\hline $08005-2356$ & & F5Ie & F5e & AA \\
\hline $08143-4406$ & PM 1-39 & K12I & & AA \\
\hline 08187-1905 & & G1I & F6Ib/II & AA \\
\hline $08213-3857$ & & F2Ie & F3V & AA \\
\hline $08281-4850$ & PM 1-40 & FOI & & AA \\
\hline $09425-6040$ & & $\mathrm{C}$ & & AA \\
\hline $10256-5628$ & PM 2-11 & F5I & & AA \\
\hline $11201-6545$ & PM 1-56 & A3Ie & & AA \\
\hline $11387-6113$ & & A3Ie & & AA \\
\hline $12067-4508$ & Hen 3-755 & G1Ie & G2w... & AA \\
\hline $12145-5834$ & PM 1-64 & em & & AA \\
\hline $12302-6317$ & & $*$ & & AA \\
\hline $13010-6012$ & PM 1-72 & M2I & & $\mathrm{CA}$ \\
\hline $13203-5917$ & & G2I & & AA \\
\hline $13245-5036$ & & A79Ie & & AA \\
\hline $13266-5551$ & & B1Ie & $\mathrm{O}+\ldots$ & AA \\
\hline $13313-5838$ & & K5I & K1III & AA \\
\hline $13416-6243$ & & $*$ & & AA \\
\hline $13428-6232$ & PM 2-14 & em & & AA \\
\hline $14325-6428$ & & F5I & & AA \\
\hline $14331-6435$ & Hen 3-1013 & B8Ie & B3Iab:e & AA \\
\hline $14341-6211$ & PM 1-85 & $*$ & & $\mathrm{BA}$ \\
\hline $14346-5952$ & & em & & AA \\
\hline $14429-4539$ & & F4Ie & M & AA \\
\hline $14482-5725$ & PM 1-86 & A2I & & AA \\
\hline $14488-5405$ & & A0Ie & & AA \\
\hline $15039-4806$ & & $\mathrm{~A} 0 \mathrm{I}$ & A1/A2Ib/ & AA \\
\hline $15210-6554$ & PM 2-16 & K2I & & $\mathrm{BA}$ \\
\hline $15310-6149$ & & A7I & F0V & AA \\
\hline $15406-4946$ & & M4II & & AA \\
\hline $15482-5741$ & & F7I & & AA \\
\hline $16206-5956$ & & A3Ie & A3Iab:e & AA \\
\hline $16279-4757$ & PM 2-18 & M3II & & AA \\
\hline $16283-4424$ & PM 2-19 & A2Ie & & AA \\
\hline $16476-1122$ & & M1I & & AA \\
\hline
\end{tabular}


Table 4. continued.

\begin{tabular}{|c|c|c|c|c|}
\hline IRAS name & Other identifications & $\begin{array}{c}\text { Spectral type } \\
\text { our survey }\end{array}$ & $\begin{array}{c}\text { Spectral type } \\
\text { (SIMBAD) }\end{array}$ & $\begin{array}{c}\begin{array}{c}\text { Reliability } \\
\text { code }^{\dagger}\end{array} \\
\end{array}$ \\
\hline $16494-3930$ & & G2I & & $\mathrm{CA}$ \\
\hline $16552-3050$ & PM 1-120 & K0I & & AA \\
\hline $17106-3046$ & PM 2-23 & F5I & & AA \\
\hline $17195-2710$ & PN G358.7+05.1 & $\mathrm{em}$ & & AA \\
\hline $17203-1534$ & & A0Ie & B1IIIpe & AA \\
\hline $17208-3859$ & RPZM 14 & A2I & & AA \\
\hline $17223-2659$ & PM 2-26 & M5III & & AA \\
\hline $17245-3951$ & PM 2-27 & F6I & & AA \\
\hline $17253-2831$ & & M4II & & AA \\
\hline $17287-3443$ & & $*$ & & $\mathrm{CA}$ \\
\hline $17300-3509$ & PM 1-156 & G2I & & AA \\
\hline $17310-3432$ & PM 1-157 & A2I & & AA \\
\hline $17317-2743$ & PM 1-158 & F5I & & $\mathrm{CA}$ \\
\hline $17332-2215$ & PM 1-160 & K2I & & AA \\
\hline $17364-1238$ & PM 1-167 & $\mathrm{em}$ & & $\mathrm{BB}$ \\
\hline $17370-3357$ & PM 1-169 & G3I & & AA \\
\hline 17376-2040 & & F6I & & BA \\
\hline $17388-2203$ & & G0I & & AA \\
\hline $17392-3020$ & & $*$ & & AA \\
\hline $17436+5003$ & & A7I & F3Ib & AA \\
\hline $17441-2411$ & & F4I & F5: & AA \\
\hline $17476-4446$ & PM 2-29 & B7I & & AA \\
\hline $17488-1741$ & PM 1-184 & F7I & & BA \\
\hline $17516-2526$ & & $\mathrm{em}$ & & $\mathrm{AB}$ \\
\hline $17542-0603$ & PM 2-32 & $\mathrm{em}$ & $\mathrm{Ge}$ & AA \\
\hline $17576-2653$ & PM 1-198 & A7I & & BA \\
\hline $17579-3121$ & RPZM 44 PM 2-33 & F4I & & AA \\
\hline $18019-3403$ & & B8I & & AA \\
\hline $18025-3906$ & PM 2-34 & G1I & $\mathrm{G} 2$ & AA \\
\hline $18044-1303$ & PM 1-212 & F7I & & AA \\
\hline $18075-0924$ & & G2I & & AA \\
\hline $18096-3230$ & PM 1-218 & G3I & & AA \\
\hline $18420-0512$ & PM $1-255$ & M1I & & AA \\
\hline $18582+0001$ & PM 1-272 & K2I & & AA \\
\hline $19024+0044$ & & $\mathrm{em}$ & & AA \\
\hline 19114+0002 & & F7I & G5Ia & AA \\
\hline $19200+3457$ & PM 1-300 & $\mathrm{Fe}$ & B... & AA \\
\hline $19207+2023$ & & F6I & & AA \\
\hline $19225+3013$ & & M2II & & AA \\
\hline $19306+1407$ & & G5I & B0:e & AA \\
\hline $19356+0754$ & & K2I & & AA \\
\hline $19386+0155$ & & F5I & $\mathrm{F}$ & AA \\
\hline $19422+1438$ & PM 1-312 & F5I & & AA \\
\hline $19477+2401$ & & F4I-F7I & & AA \\
\hline 19500-1709 & & F0Ie & F2/F3Iab & AA \\
\hline $19589+4020$ & PM 1-315 & F5I & & AA \\
\hline $20160+2734$ & & F3Ie & & AA \\
\hline $20259+4206$ & & F3I & & AA \\
\hline $20572+4919$ & & F3Ie & $\mathrm{Fe}$ & AA \\
\hline $21289+5815$ & & A2Ie & & AA \\
\hline
\end{tabular}


O. Suárez et al.: Spectroscopic atlas of post-AGB and PNe, Online Material p 12

Table 4. continued.

\begin{tabular}{lcccc}
\hline \hline IRAS name & Other identifications & $\begin{array}{c}\text { Spectral type } \\
\text { our survey }\end{array}$ & $\begin{array}{c}\text { Spectral type } \\
\text { (SIMBAD) }\end{array}$ & $\begin{array}{c}\text { Reliability } \\
\text { code }^{\dagger}\end{array}$ \\
\hline $22036+5306$ & em & & AA \\
$22223+4327$ & F7I & G0Ia & AA \\
\hline
\end{tabular}

$\dagger$ This code rates our confidence in the data presented, with A representing the maximum reliability. The first letter marks our confidence in the correct identification of the optical counterpart, and the second letter indicates how confident we are in the evolutionary classification assigned.

* Faint and red continuum. 
O. Suárez et al.: Spectroscopic atlas of post-AGB and PNe, Online Material $p 13$

Table 5. Main characteristics derived for the IRAS sources identified as transition objects.

\begin{tabular}{|c|c|c|c|c|c|}
\hline IRAS name & Other names & $\begin{array}{l}\text { Spectral type } \\
\text { our survey }\end{array}$ & $\begin{array}{c}\text { Spectral type } \\
\text { (SIMBAD) }\end{array}$ & $\begin{array}{c}\text { Reliability } \\
\text { code }^{\dagger}\end{array}$ & Morphology \\
\hline $10178-5958$ & PN G285.1-02.7 Hen 3-401 & $\overline{\mathrm{BeI}}$ & $\overline{\mathrm{Be}}$ & $\overline{\mathrm{AA}}$ & Bipolar [1] \\
\hline $10197-5750$ & Hen 3-404 & A0Ie & A2Iabe & AA & Bipolar [2] \\
\hline $10215-5916$ & & A7eI & K0 & AA & \\
\hline $11353-6037$ & PM 1-60 & B5Ie & & AA & \\
\hline $11531-6111$ & PM 1-61 & B8eI & & AA & \\
\hline $15066-5532$ & PM 2-15 & & & AA & \\
\hline $16594-4656$ & PN G340.3-03.2 & $\mathrm{Ae}$ & B7 & AA & Multipolar [3] \\
\hline $17074-1845$ & Hen 3-1347 & B3eI & B3IIIe & AA & \\
\hline $17086-2403$ & PN G359.8+08.9 PM 2-22 & & G5IV-V & AA & \\
\hline $17291-2402$ & PN G002.5+05.1 PM 1-155 & & & AA & \\
\hline $17311-4924$ & PN G341.4-09.0 Hen 3-1428 & B3Ie & B1IIe & AA & \\
\hline $17347-3139$ & RPZM 28 & & & AA & Bipolar [4] \\
\hline $17423-1755$ & PN G009.3+05.7 Hen 3-1475 & & $\mathrm{Be}$ & AA & Bipolar [5] \\
\hline $17466-3031$ & RPZM 42 & & & AA & \\
\hline $18062+2410$ & PN G050.6+19.7 & B3e & B1IIIpe & AA & \\
\hline 18442-1144 & PN G022.0-04.3 & & $\mathrm{A} 3 \mathrm{~V}$ & AA & \\
\hline $19016-2330$ & PN G013.1-13.2 & & & AA & \\
\hline 19590-1249 & PN G029.1-21.2 & B2eI & B1Ibe & AA & \\
\hline $20462+3416$ & PN G076.6-05.7 & & B1Iae & AA & \\
\hline $21546+4721$ & PN G095.0-05.5 & & & AA & \\
\hline $22023+5249$ & PN G099.3-01.9 & & $\mathrm{Be}$ & AA & \\
\hline
\end{tabular}

$\dagger$ This code rates our confidence in the data presented, with A representing the maximum reliability. The first letter marks our confidence in the correct identification of the optical counterpart, and the second letter indicates how confident we are in the evolutionary classification assigned. [1] García-Lario et al. (1999a); [2] Allen et al. (1980); [3] García-Lario et al. (1999b); [4] de Gregorio-Monsalvo et al. (2004); [5] Riera et al. (1995). 


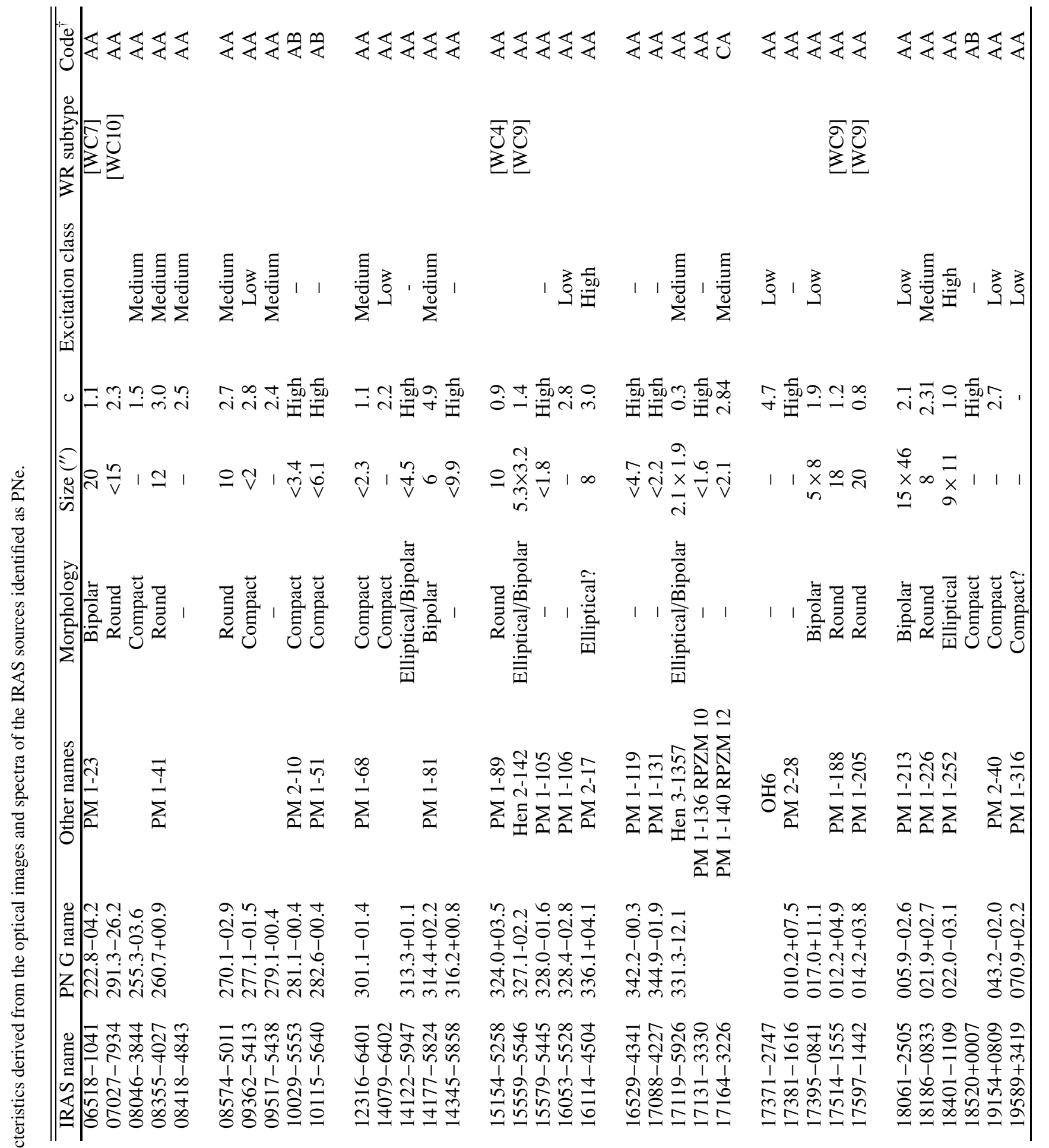


O. Suárez et al.: Spectroscopic atlas of post-AGB and PNe, Online Material p 15

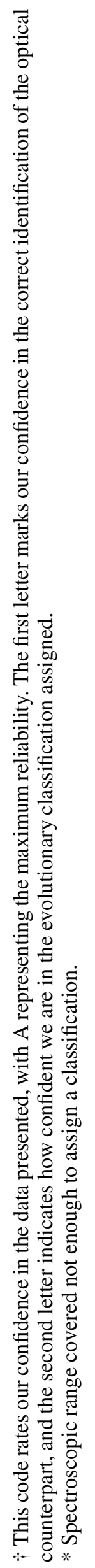


O. Suárez et al.: Spectroscopic atlas of post-AGB and PNe, Online Material p 16

\section{Appendix A: Atlas of post-AGB stars}
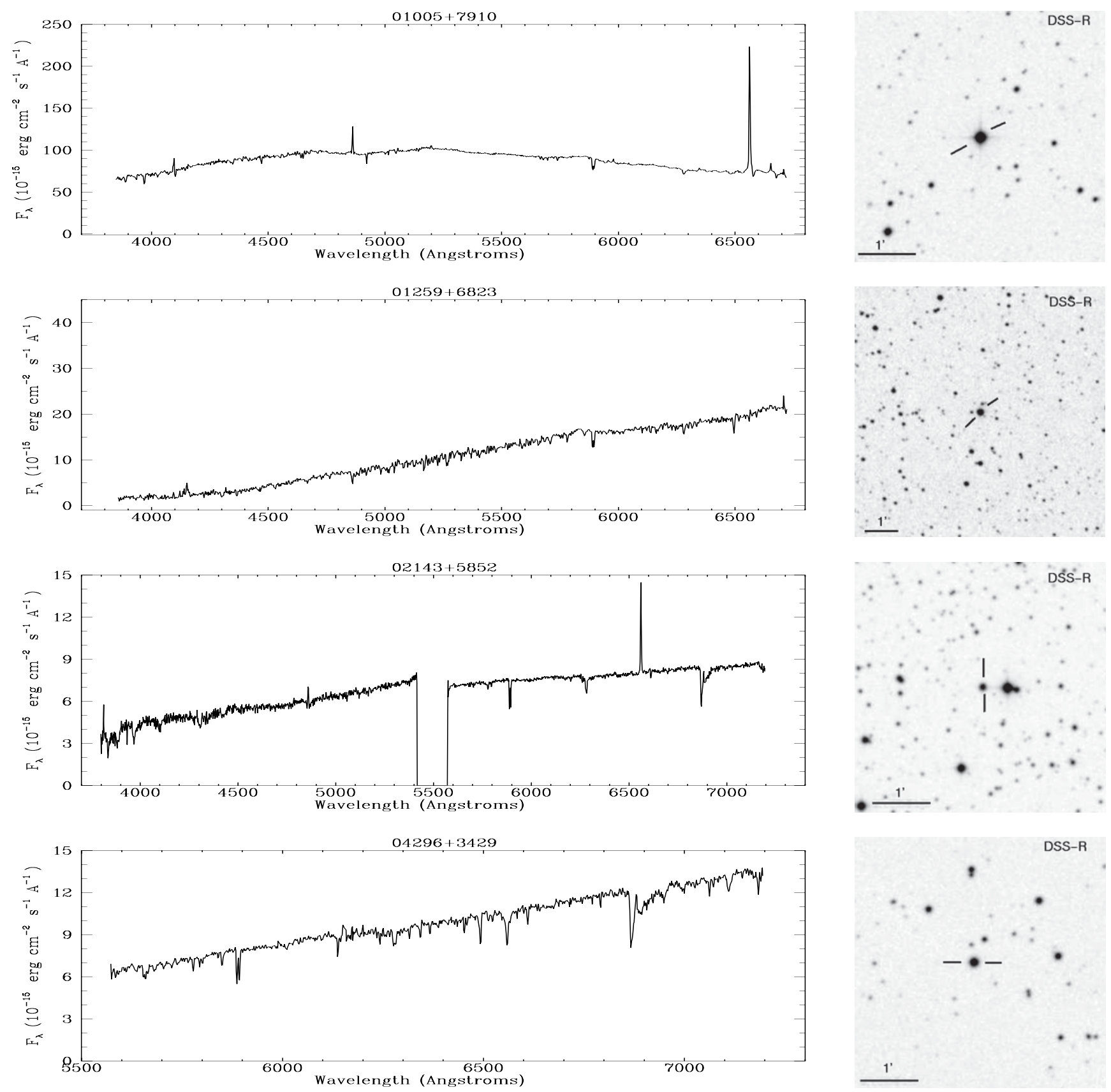

Fig. A.1. Spectra of the objects classified as post-AGB in the sample together with their corresponding identification charts. 
O. Suárez et al.: Spectroscopic atlas of post-AGB and PNe, Online Material p 17
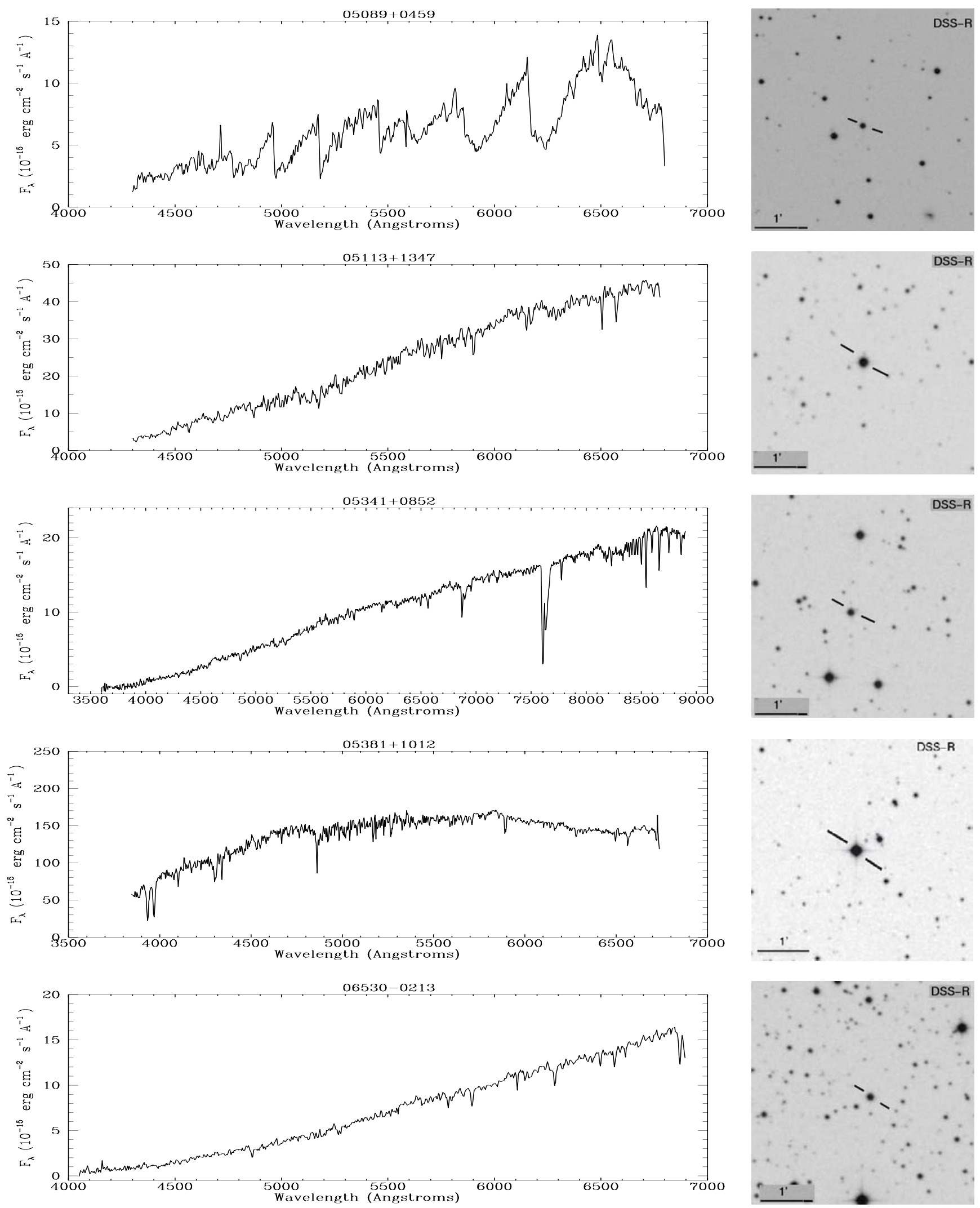

Fig. A.1. continued. 
O. Suárez et al.: Spectroscopic atlas of post-AGB and PNe, Online Material p 18
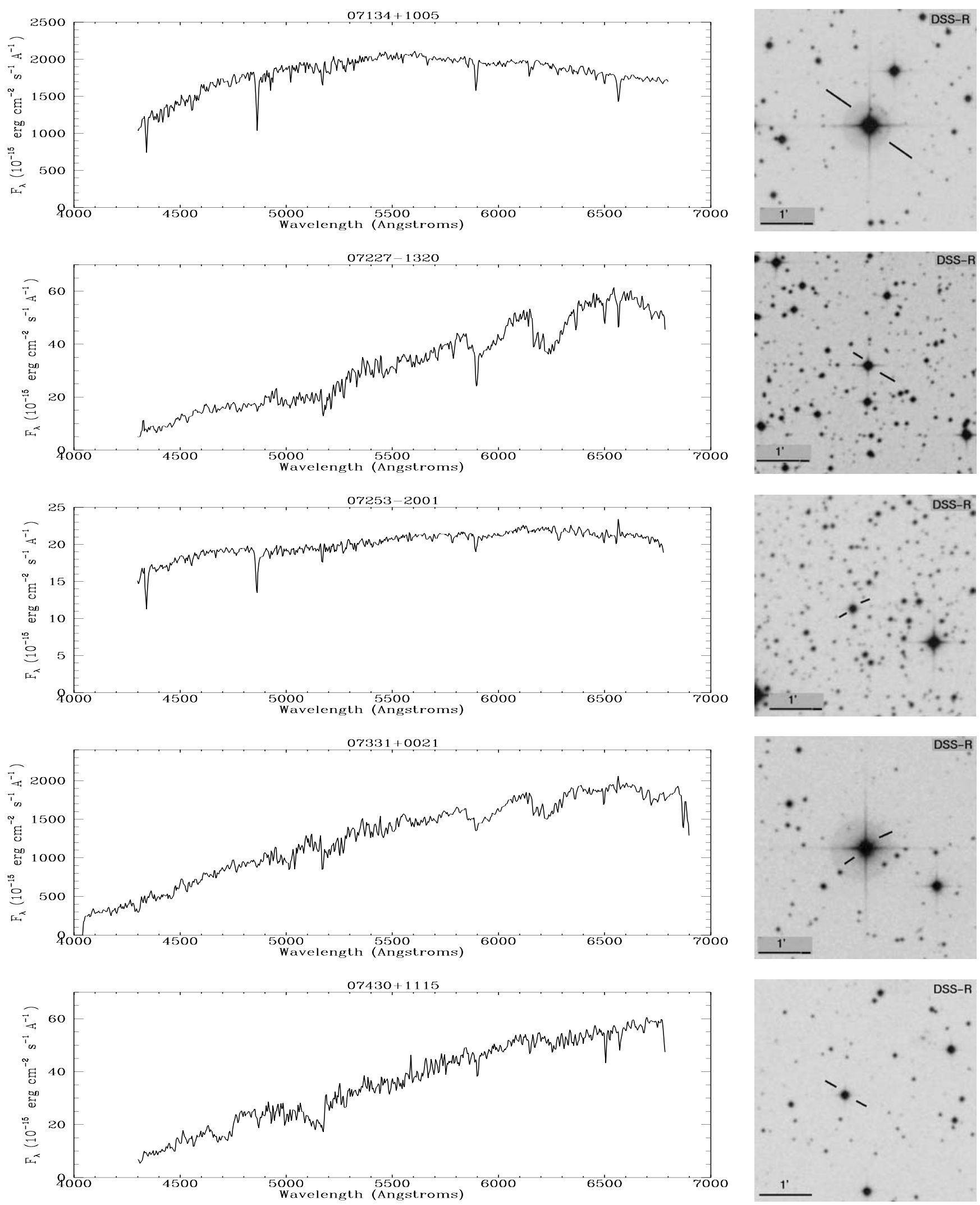

Fig. A.1. continued. 
O. Suárez et al.: Spectroscopic atlas of post-AGB and PNe, Online Material p 19
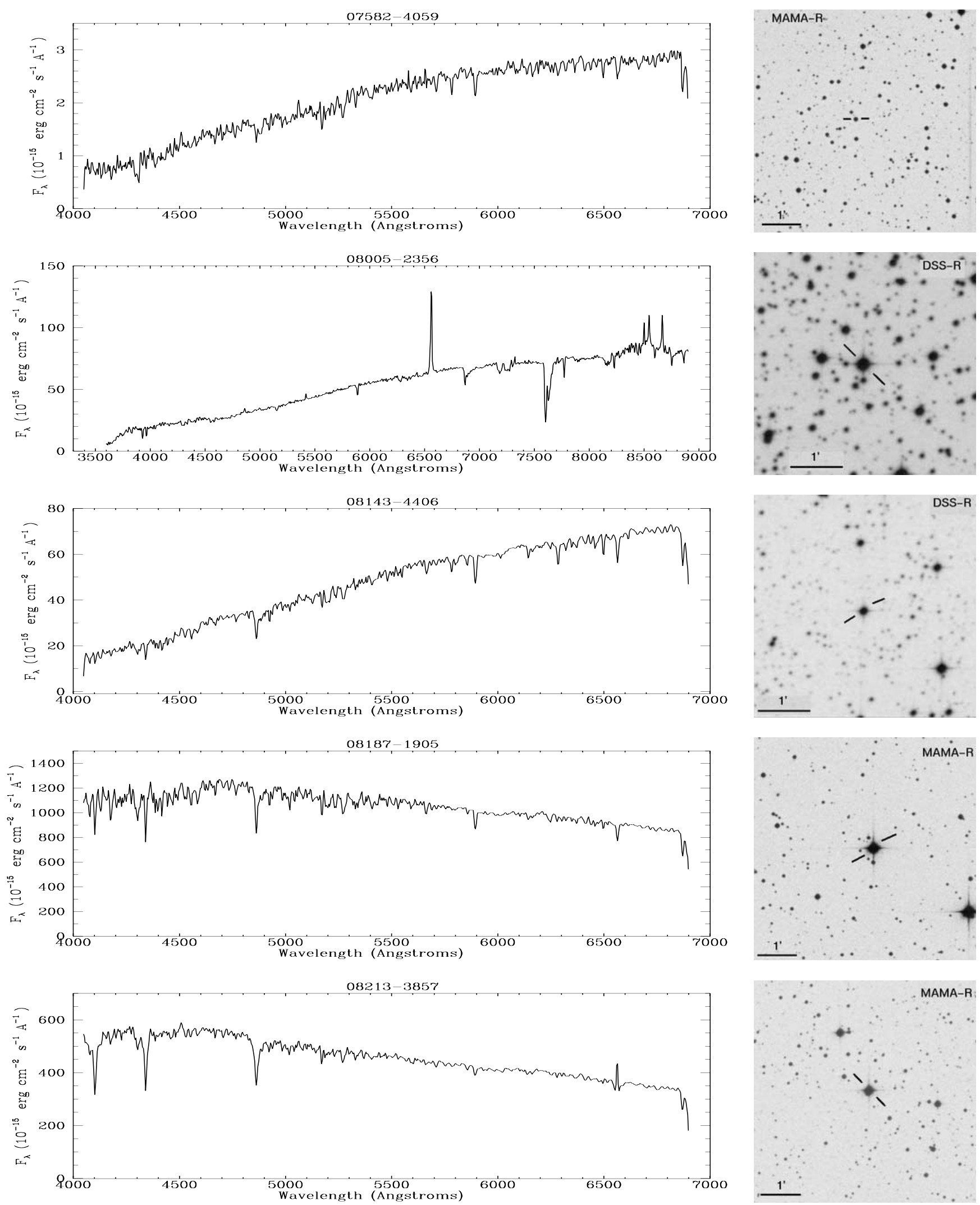

Fig. A.1. continued. 
O. Suárez et al.: Spectroscopic atlas of post-AGB and PNe, Online Material p 20
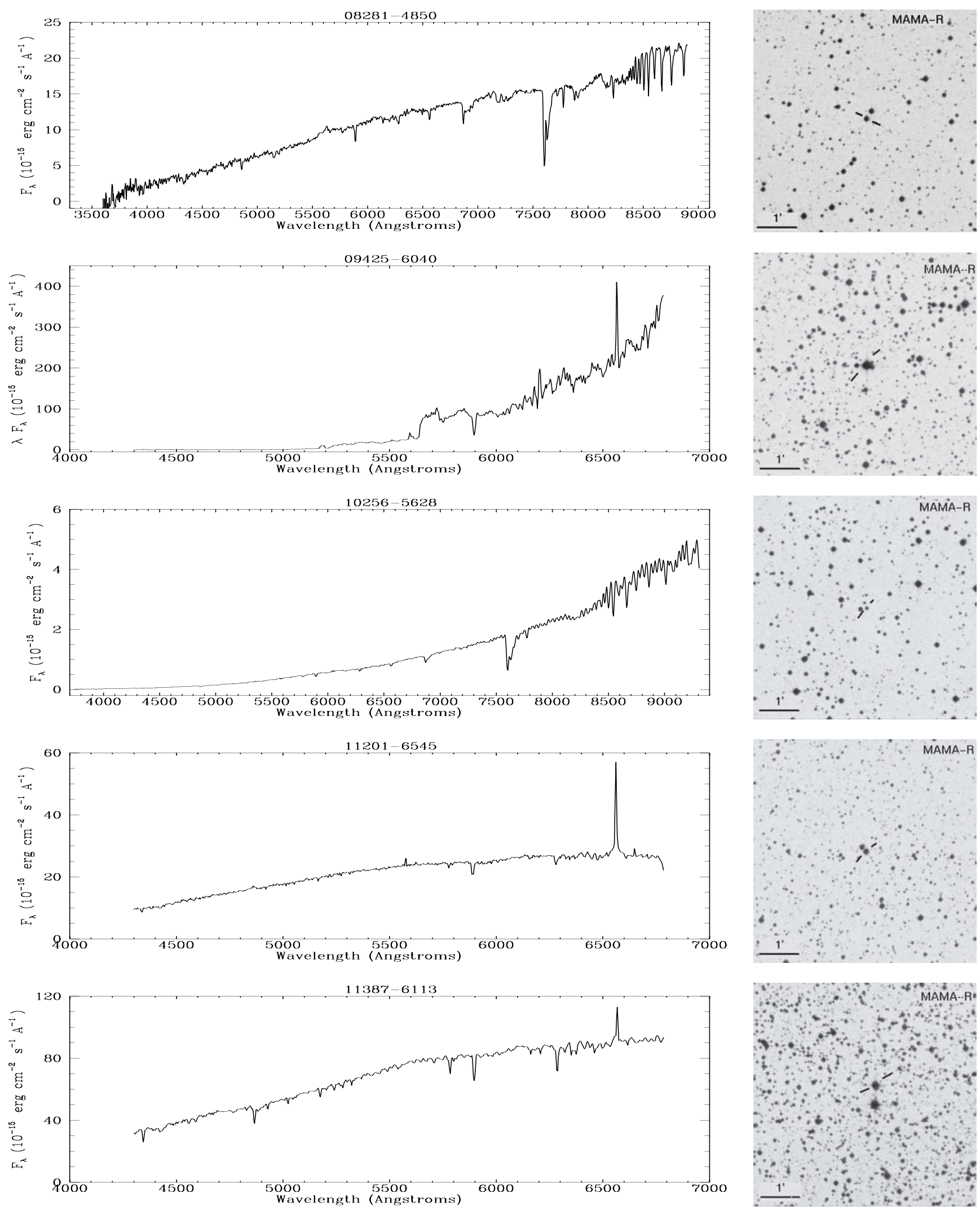

Fig. A.1. continued. 
O. Suárez et al.: Spectroscopic atlas of post-AGB and PNe, Online Material p 21
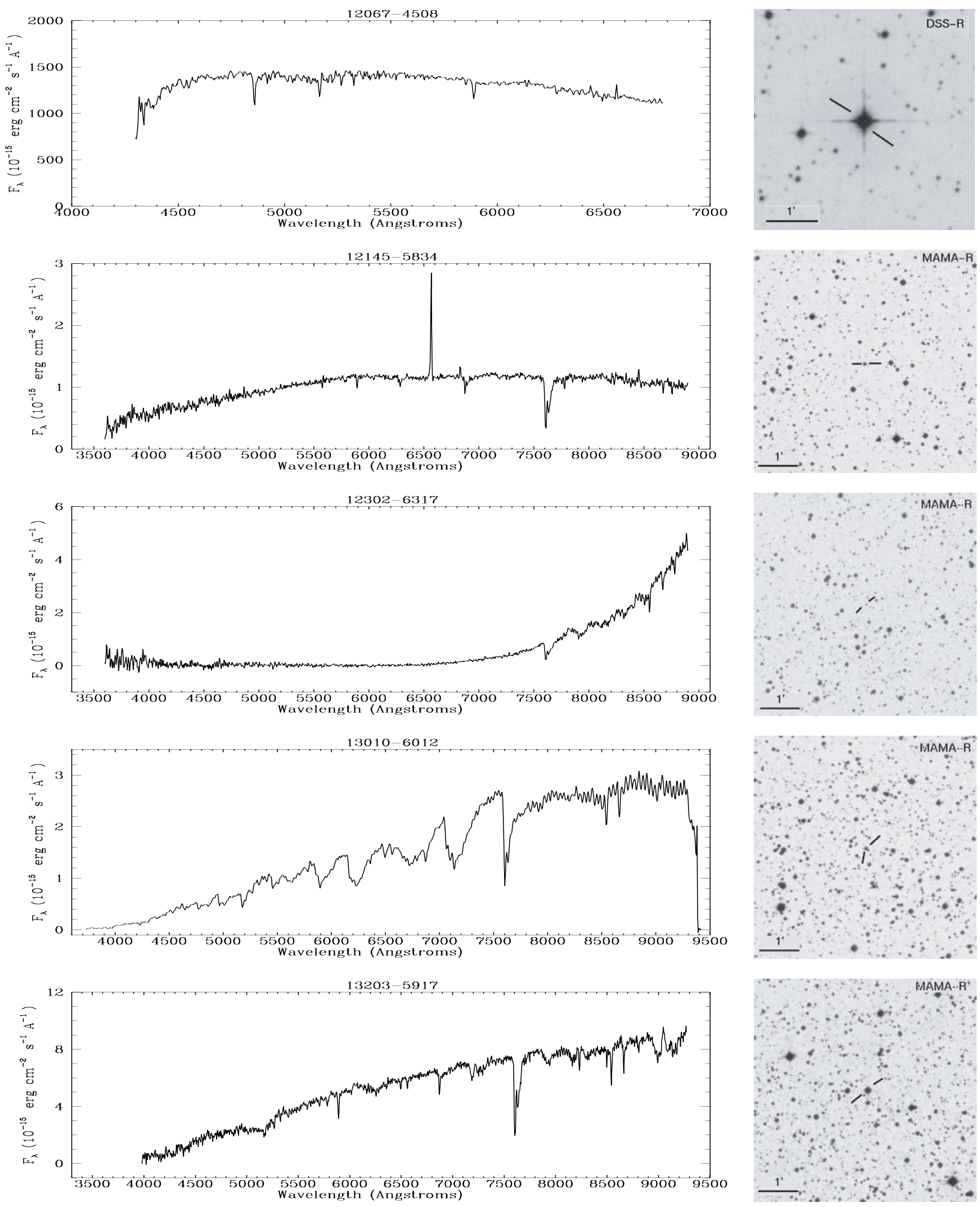

Fig. A.1. continued. 
O. Suárez et al.: Spectroscopic atlas of post-AGB and PNe, Online Material p 22
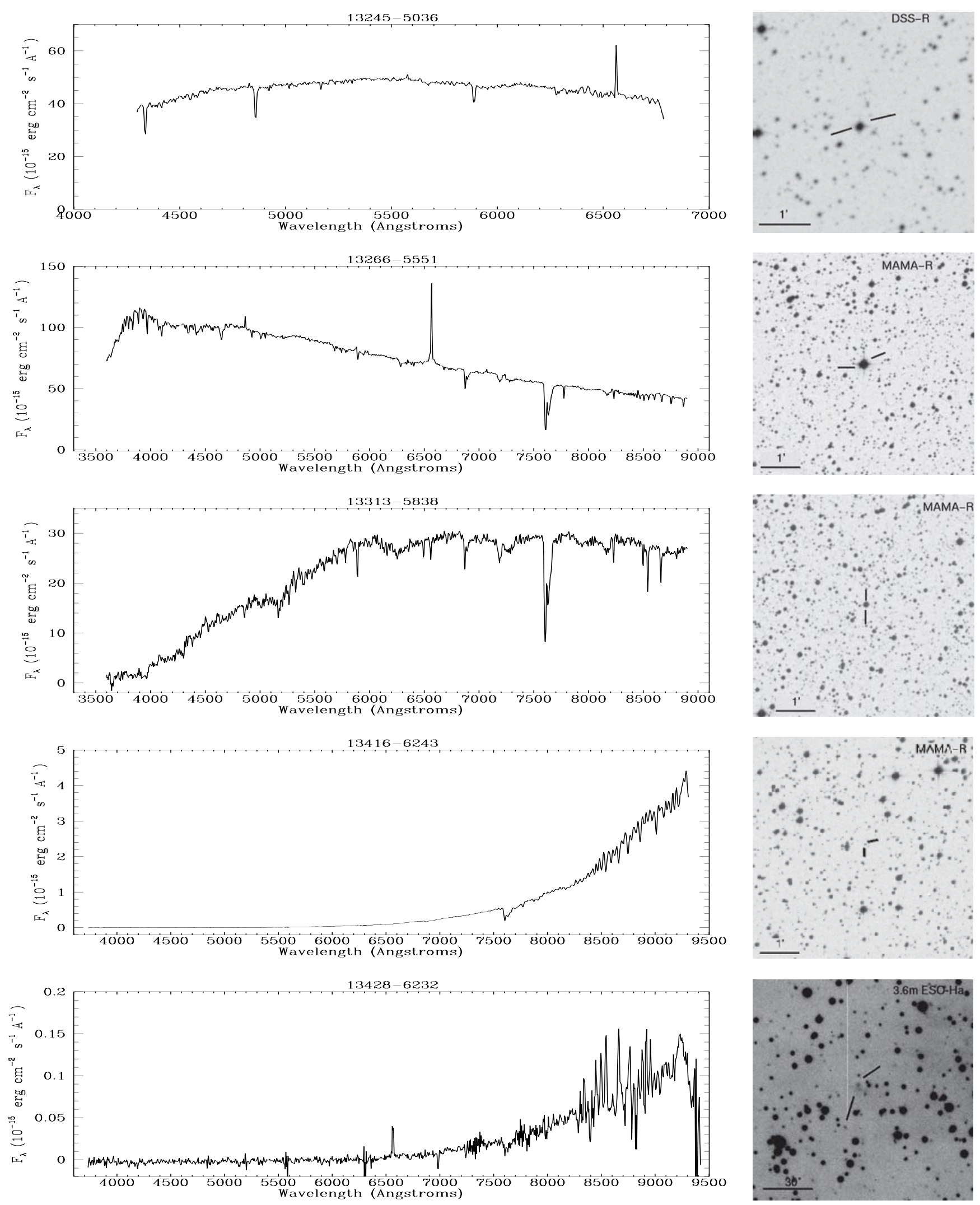

Fig. A.1. continued. 
O. Suárez et al.: Spectroscopic atlas of post-AGB and PNe, Online Material p 23
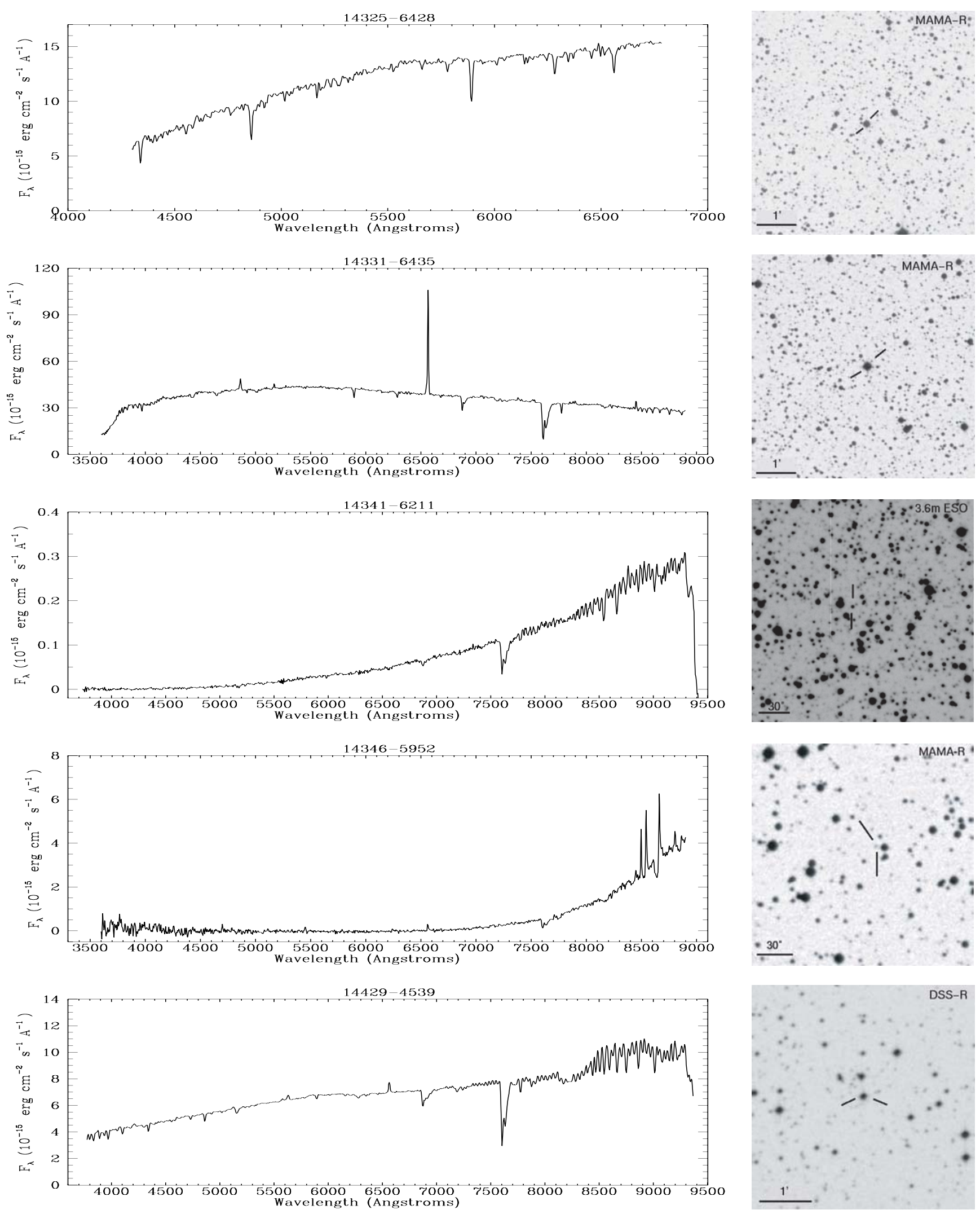

Fig. A.1. continued. 
O. Suárez et al.: Spectroscopic atlas of post-AGB and PNe, Online Material p 24
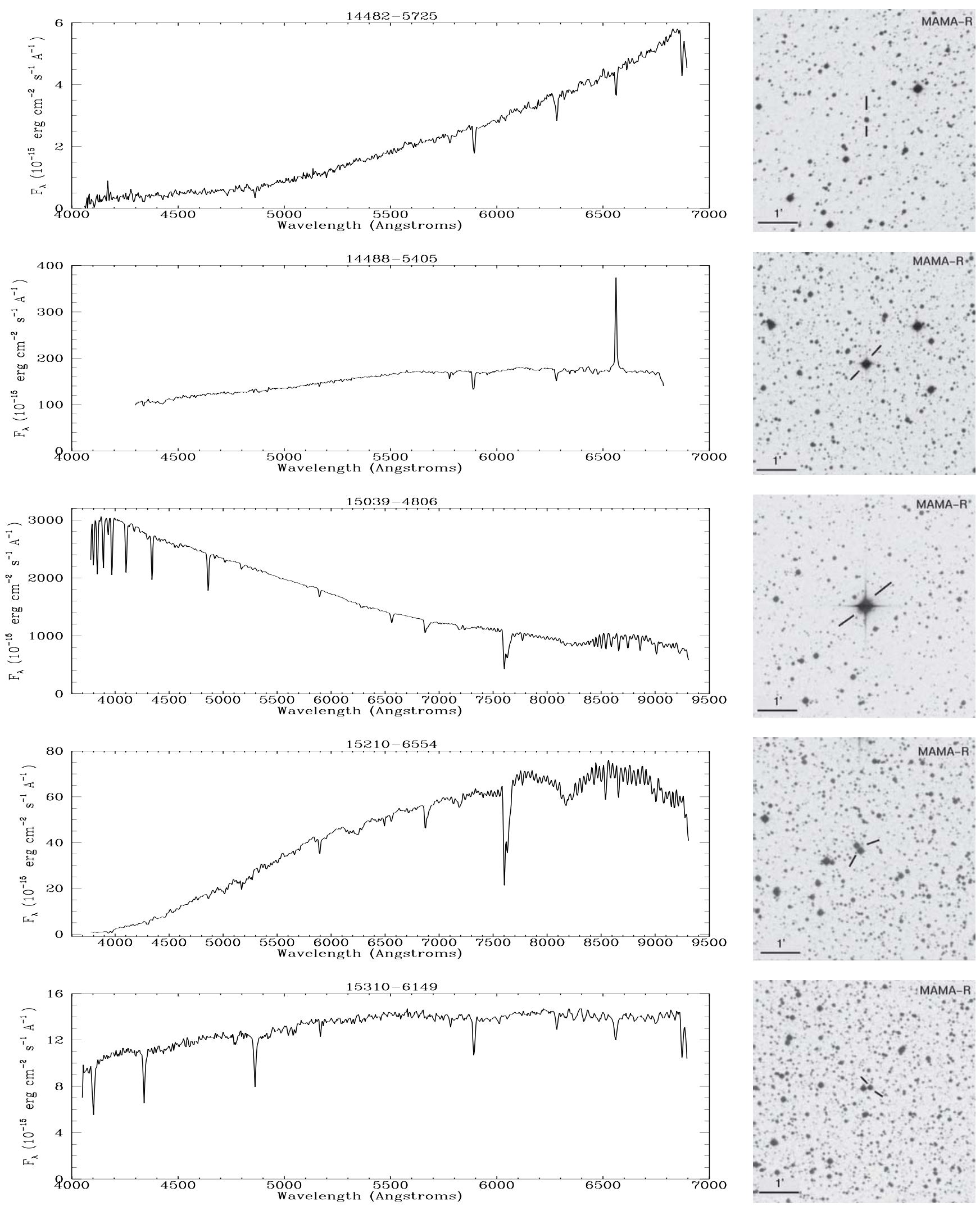

Fig. A.1. continued. 
O. Suárez et al.: Spectroscopic atlas of post-AGB and PNe, Online Material p 25
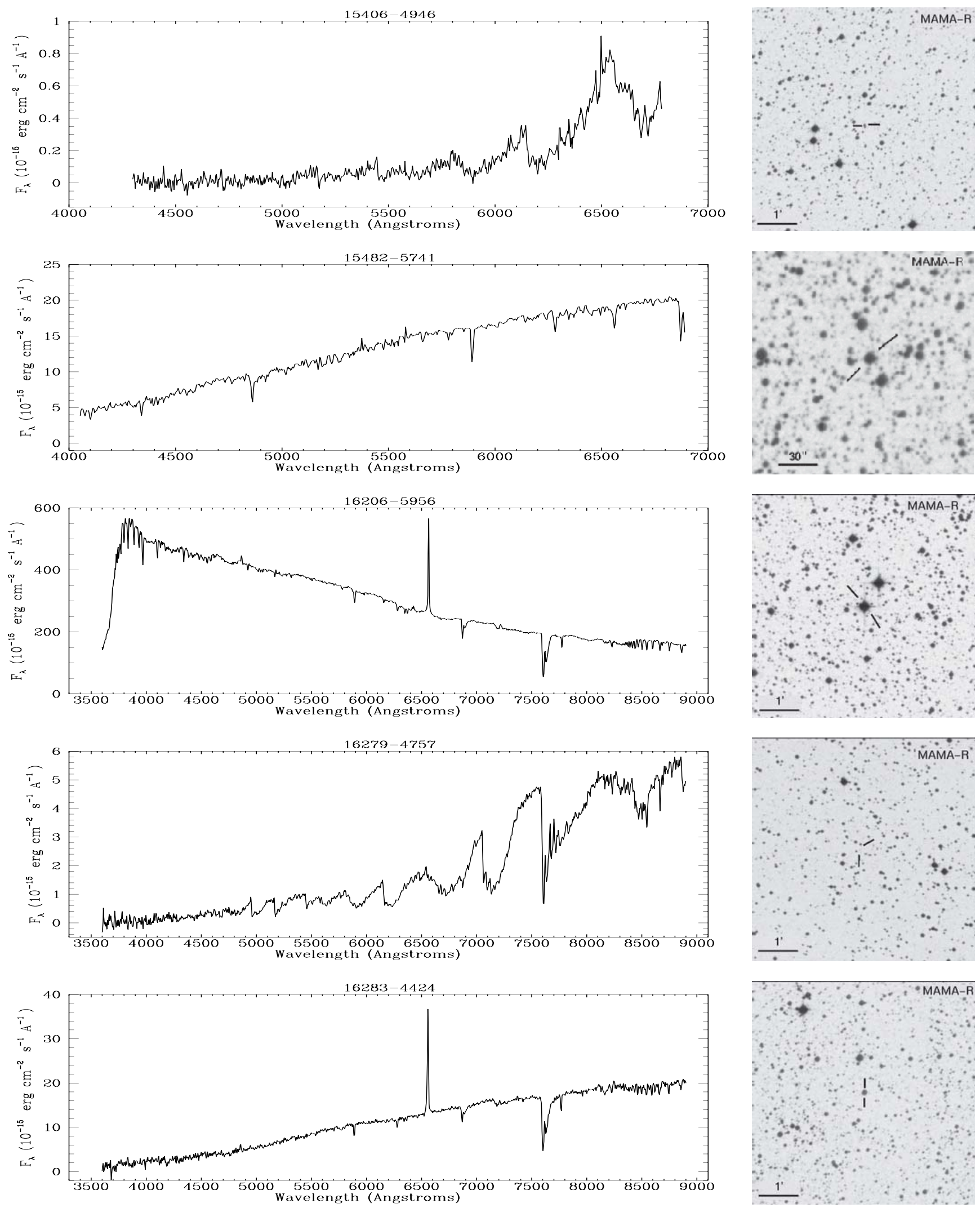

Fig. A.1. continued. 
O. Suárez et al.: Spectroscopic atlas of post-AGB and PNe, Online Material p 26
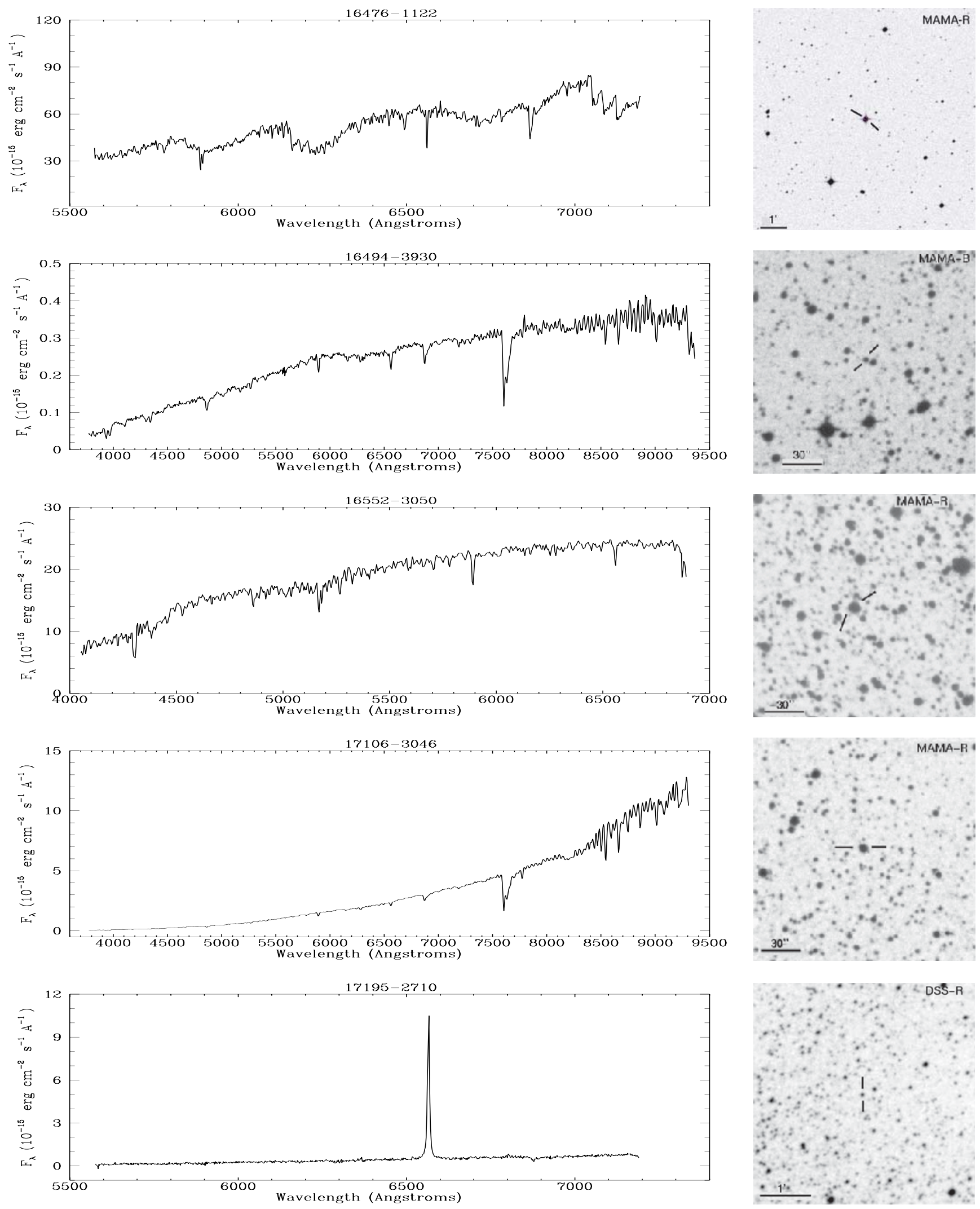

Fig. A.1. continued. 
O. Suárez et al.: Spectroscopic atlas of post-AGB and PNe, Online Material p 27
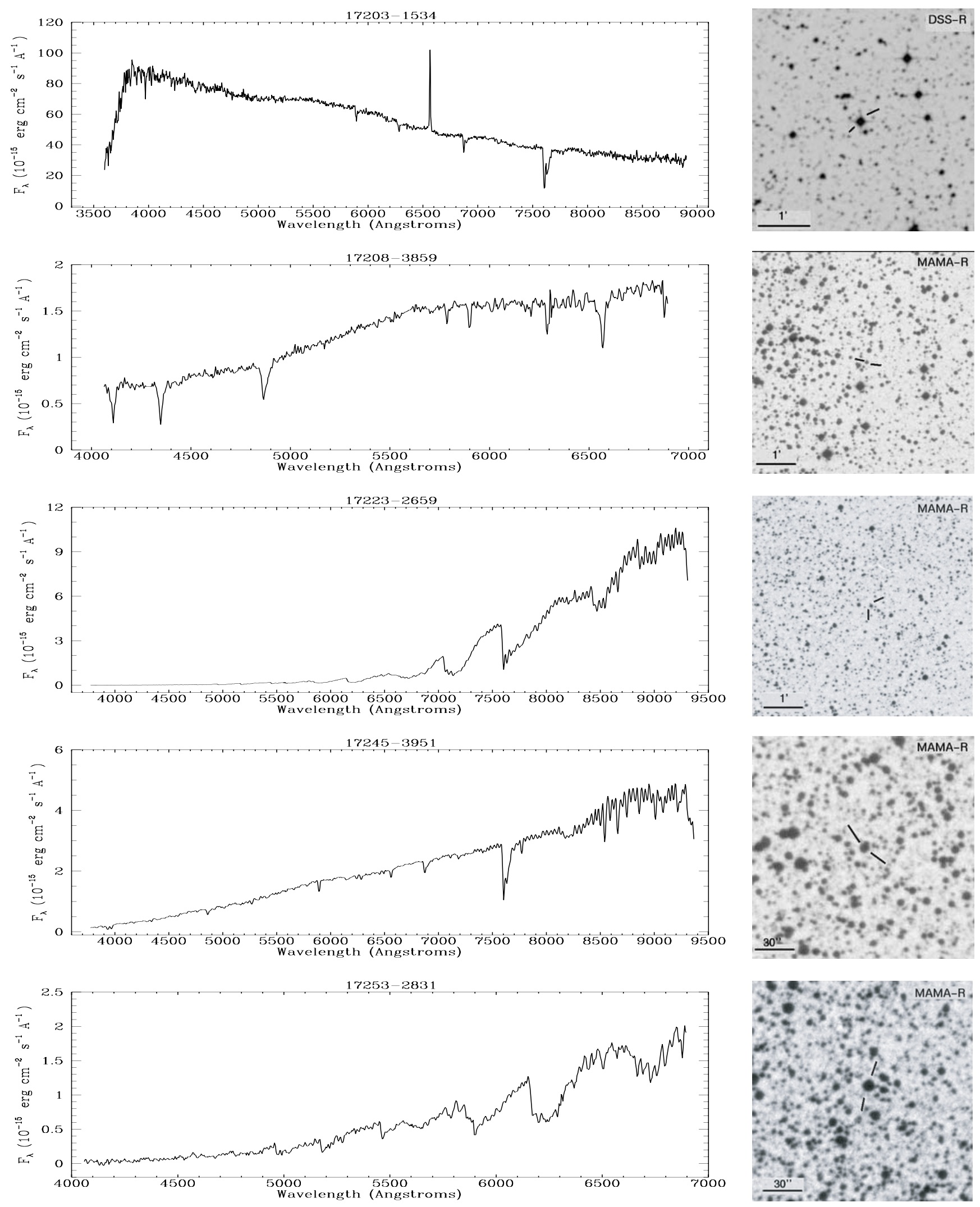

Fig. A.1. continued. 
O. Suárez et al.: Spectroscopic atlas of post-AGB and PNe, Online Material $p 28$
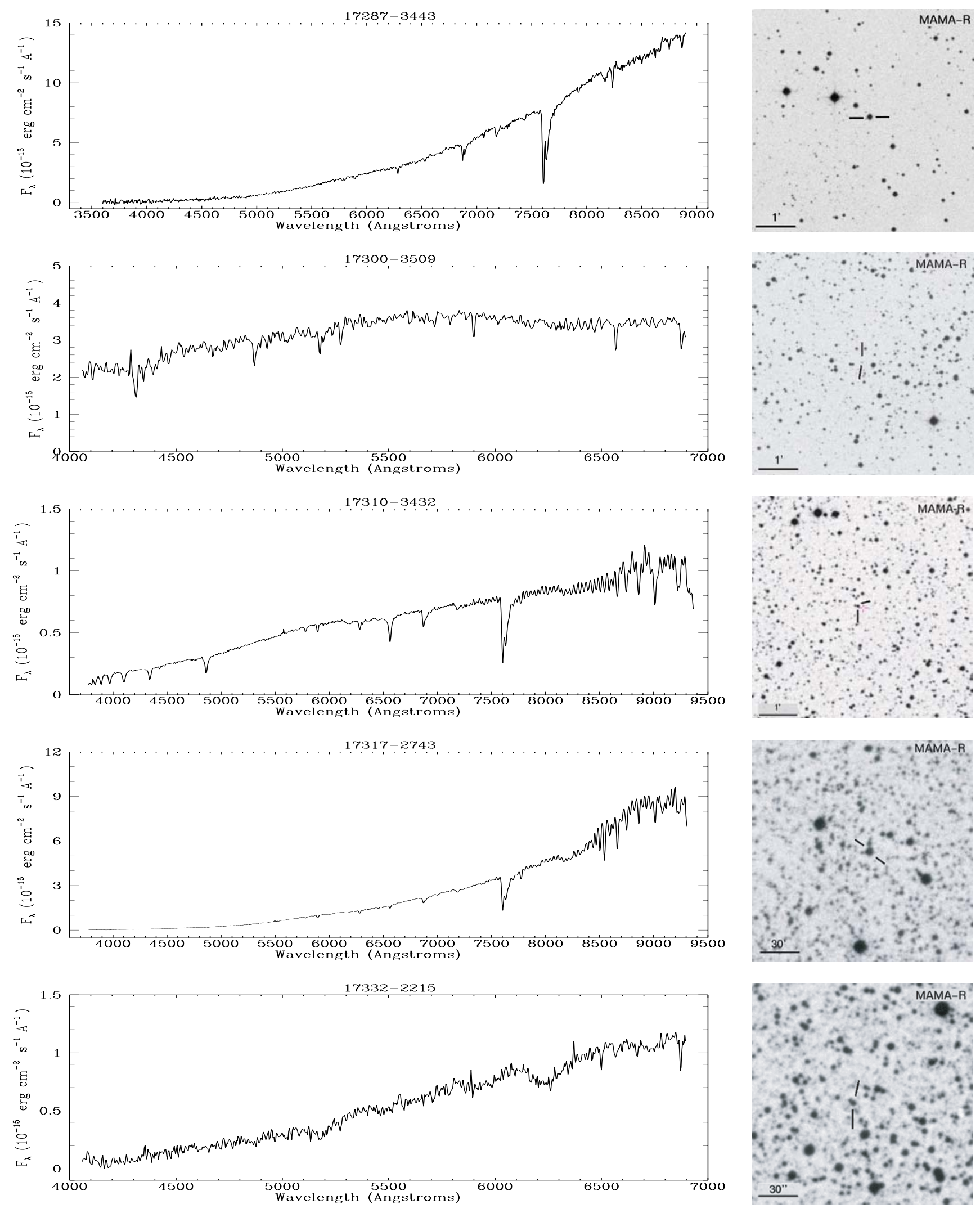

Fig. A.1. continued. 
O. Suárez et al.: Spectroscopic atlas of post-AGB and PNe, Online Material p 29
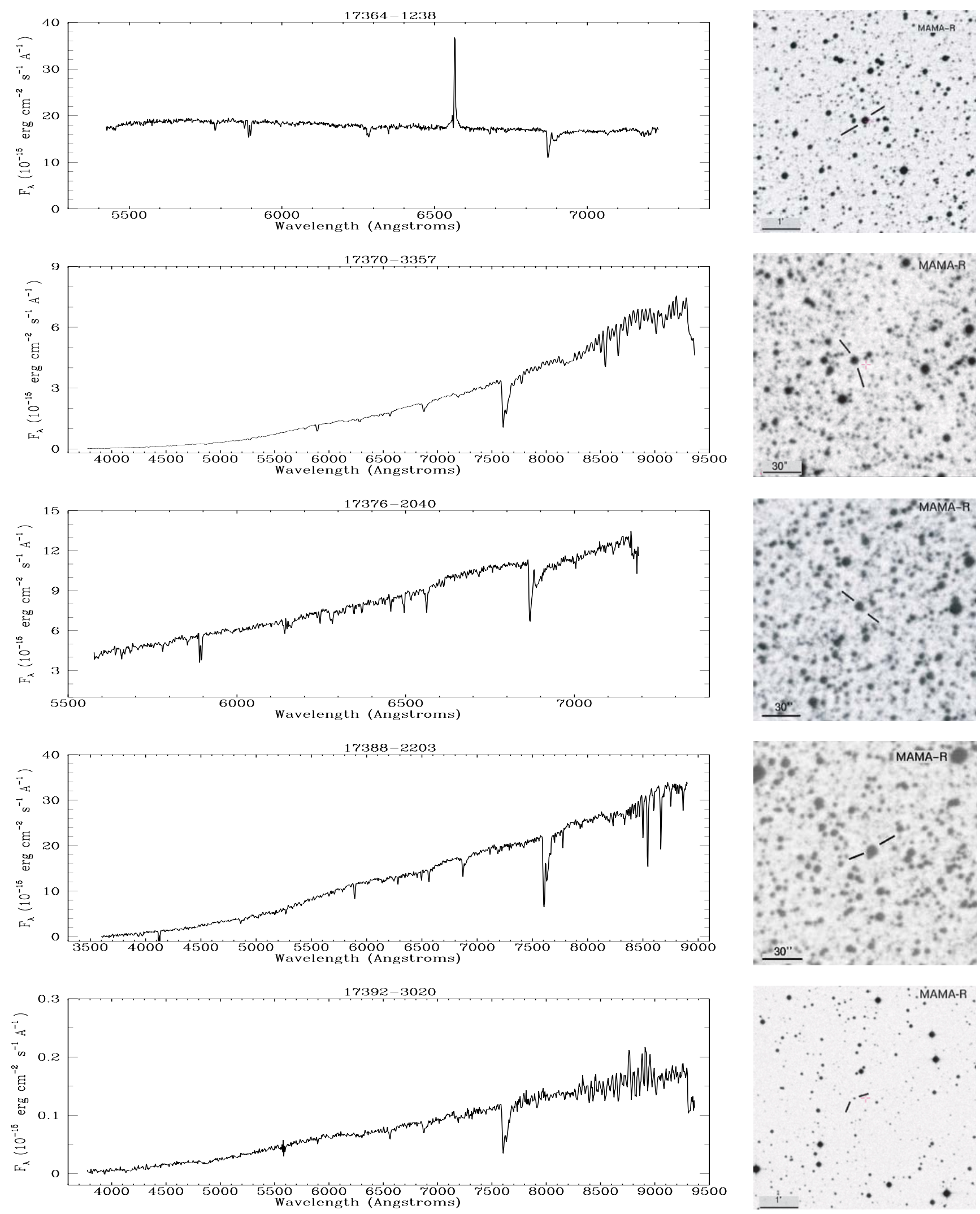

Fig. A.1. continued. 
O. Suárez et al.: Spectroscopic atlas of post-AGB and PNe, Online Material p 30
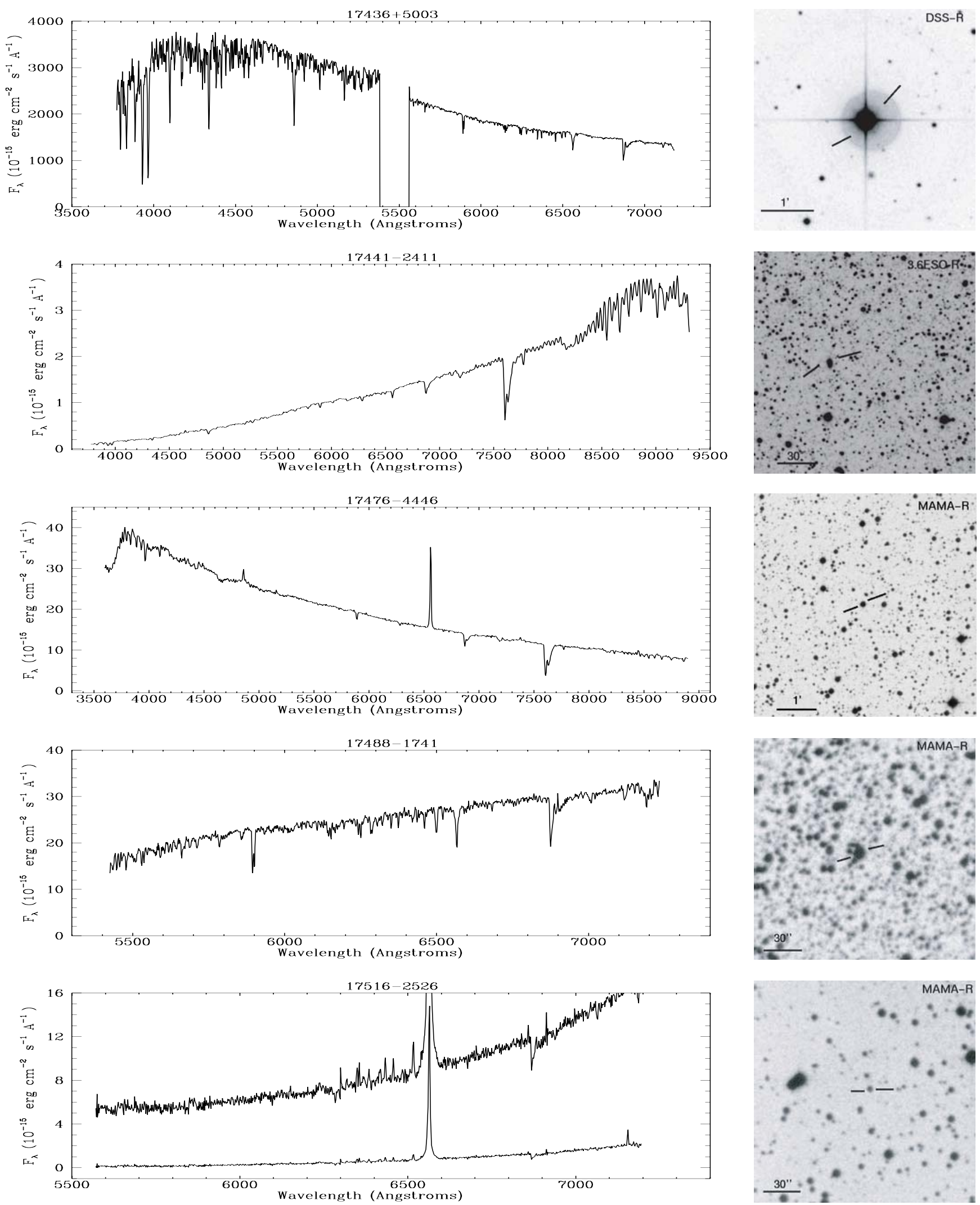

Fig. A.1. continued. 
O. Suárez et al.: Spectroscopic atlas of post-AGB and PNe, Online Material p 31
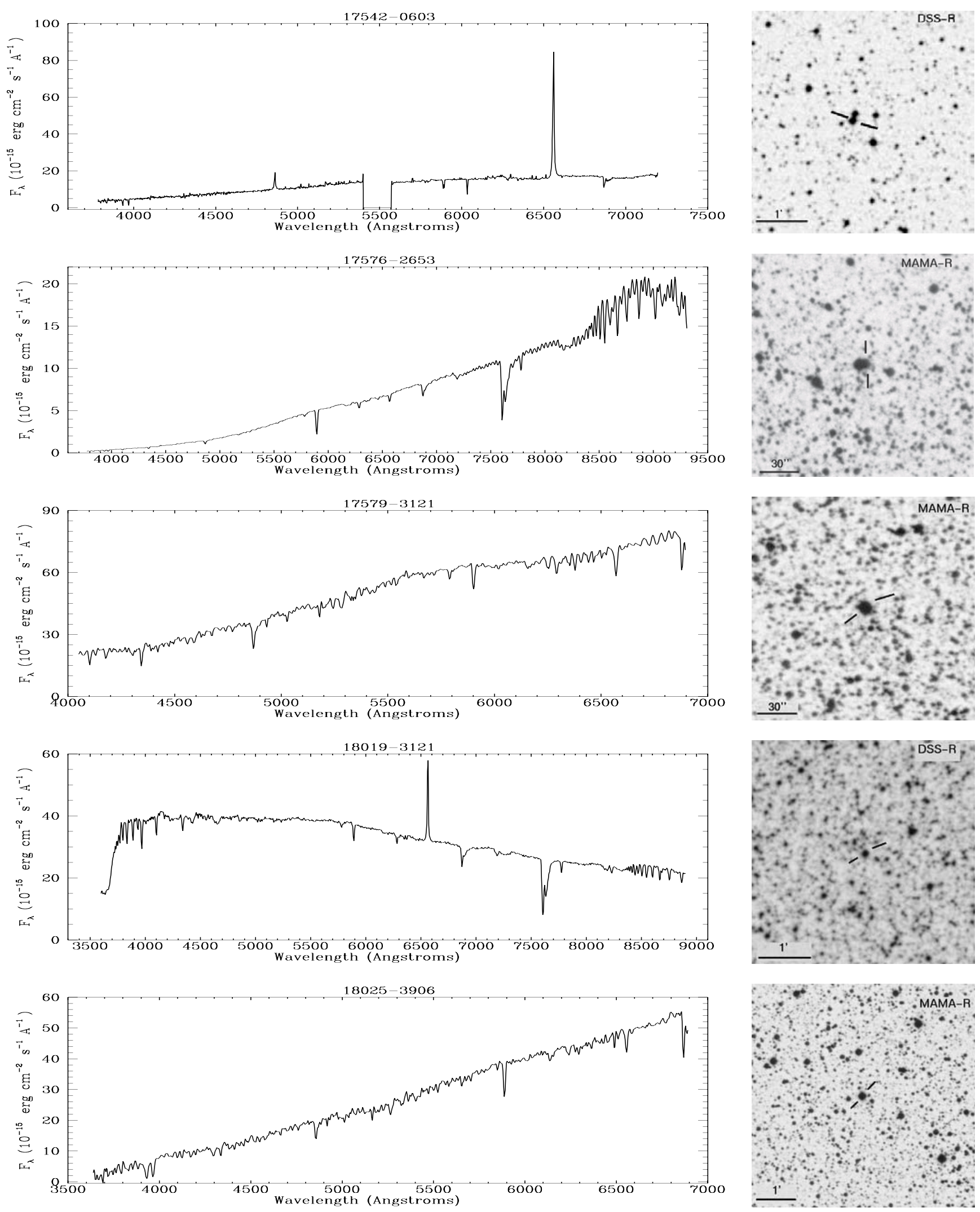

Fig. A.1. continued. 
O. Suárez et al.: Spectroscopic atlas of post-AGB and PNe, Online Material p 32
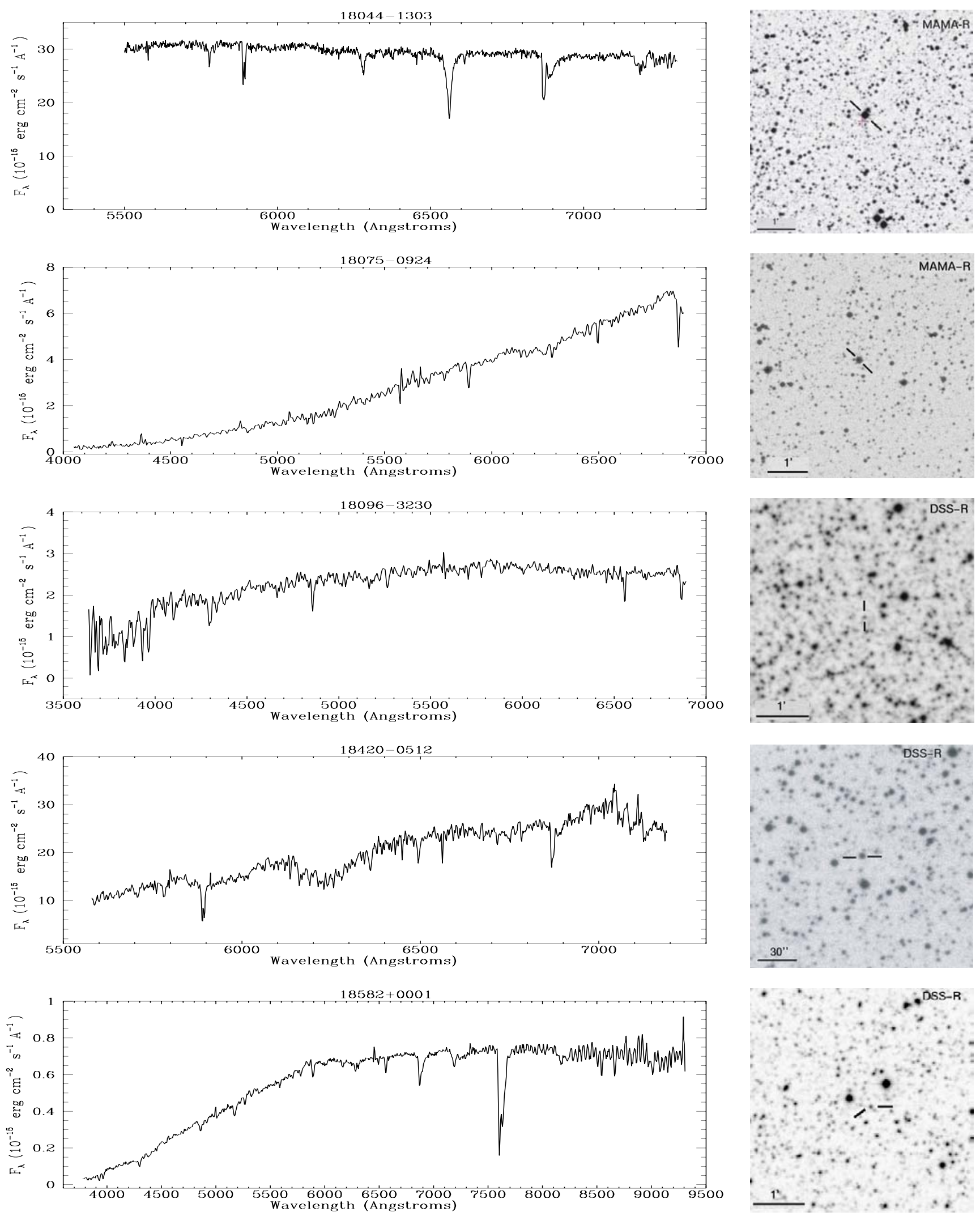

Fig. A.1. continued. 
O. Suárez et al.: Spectroscopic atlas of post-AGB and PNe, Online Material p 33
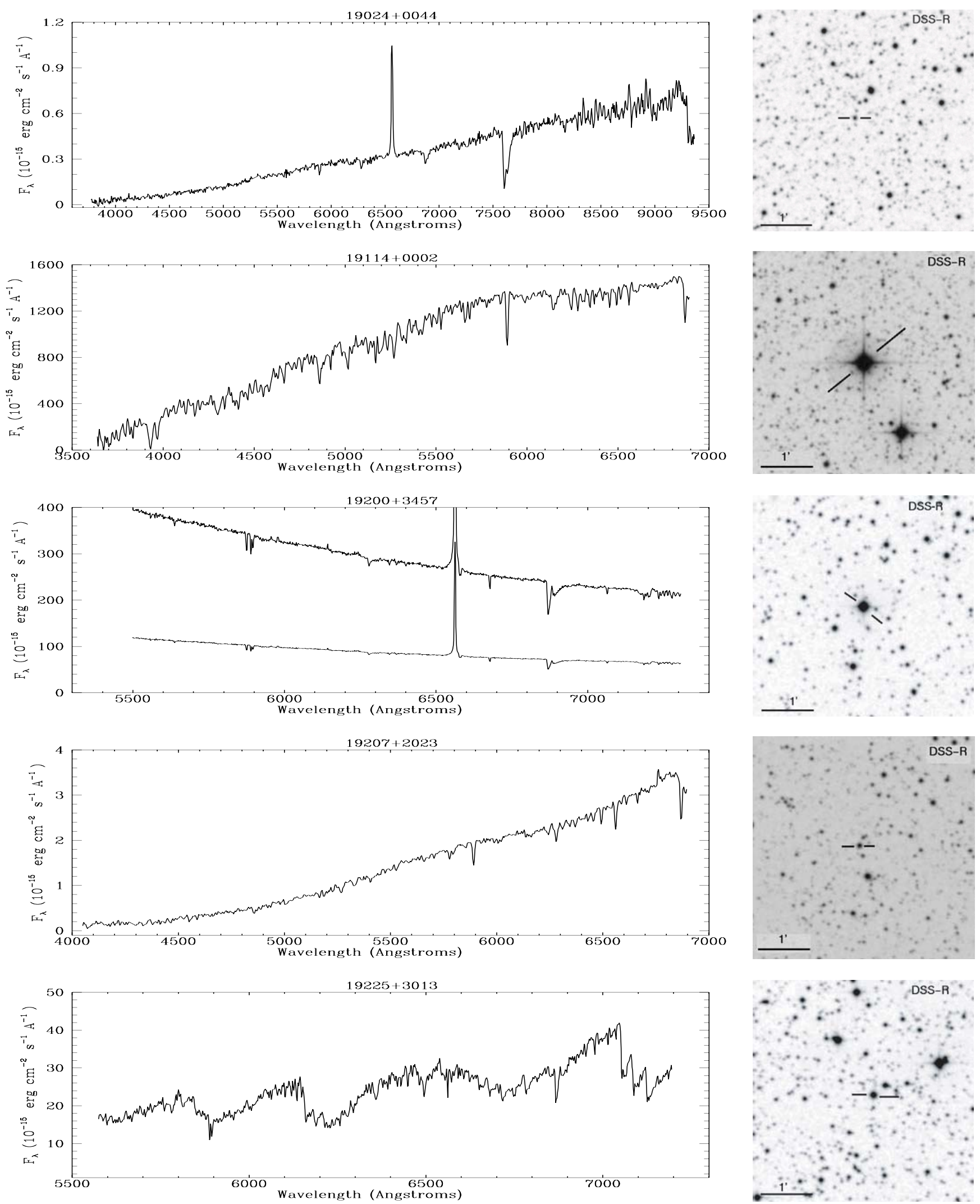

Fig. A.1. continued. 
O. Suárez et al.: Spectroscopic atlas of post-AGB and PNe, Online Material p 34
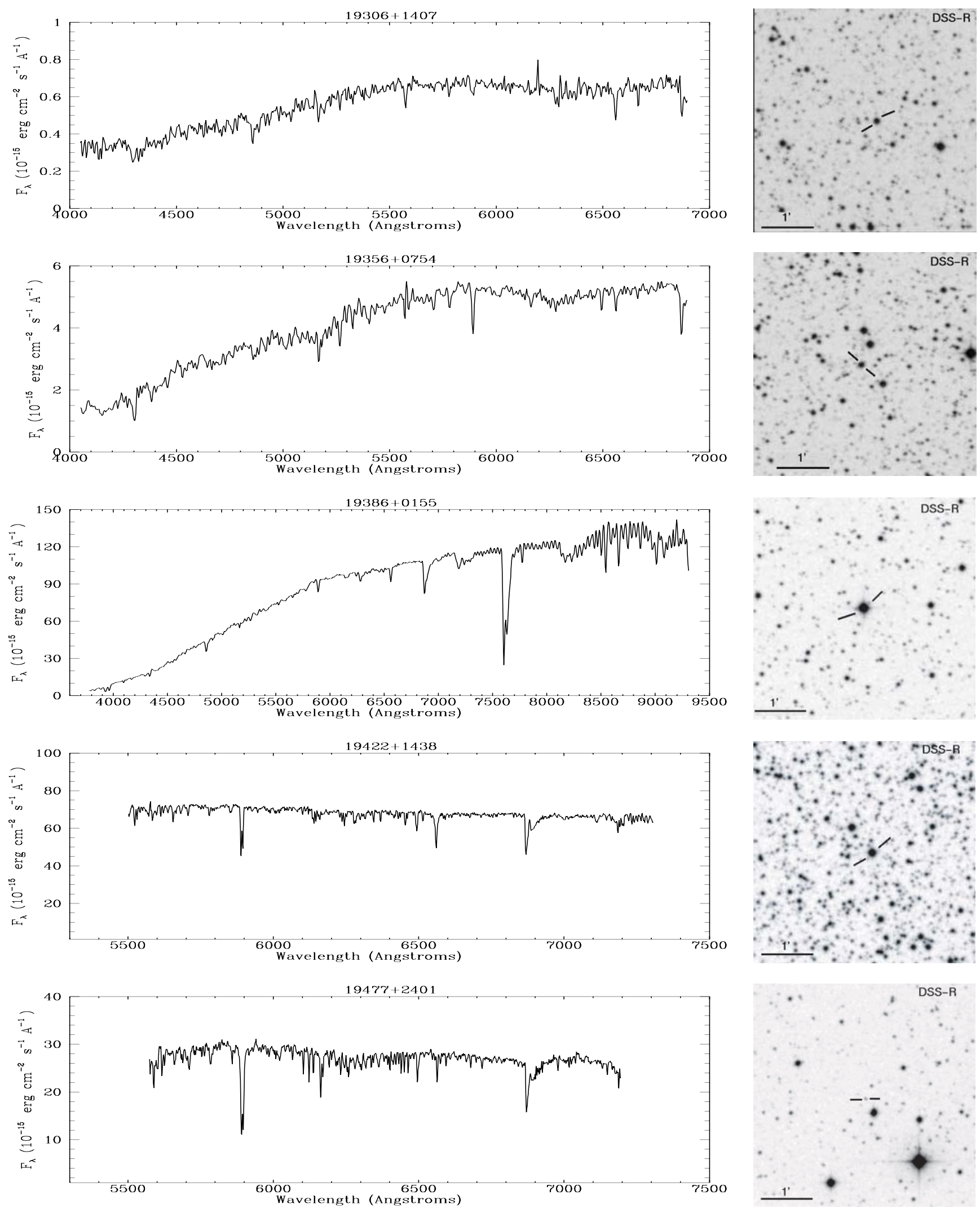

Fig. A.1. continued. 
O. Suárez et al.: Spectroscopic atlas of post-AGB and PNe, Online Material p 35
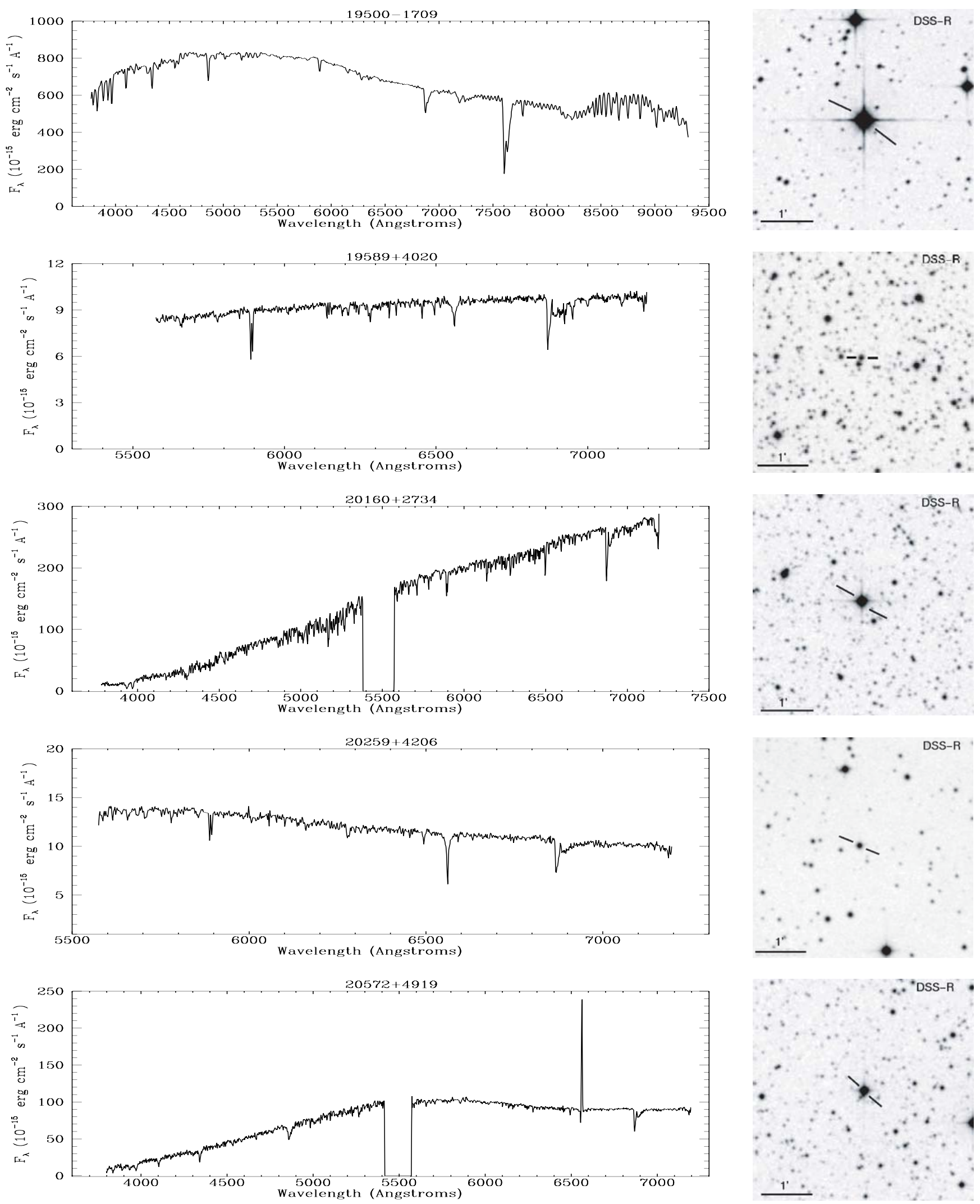

Fig. A.1. continued. 
O. Suárez et al.: Spectroscopic atlas of post-AGB and PNe, Online Material p 36
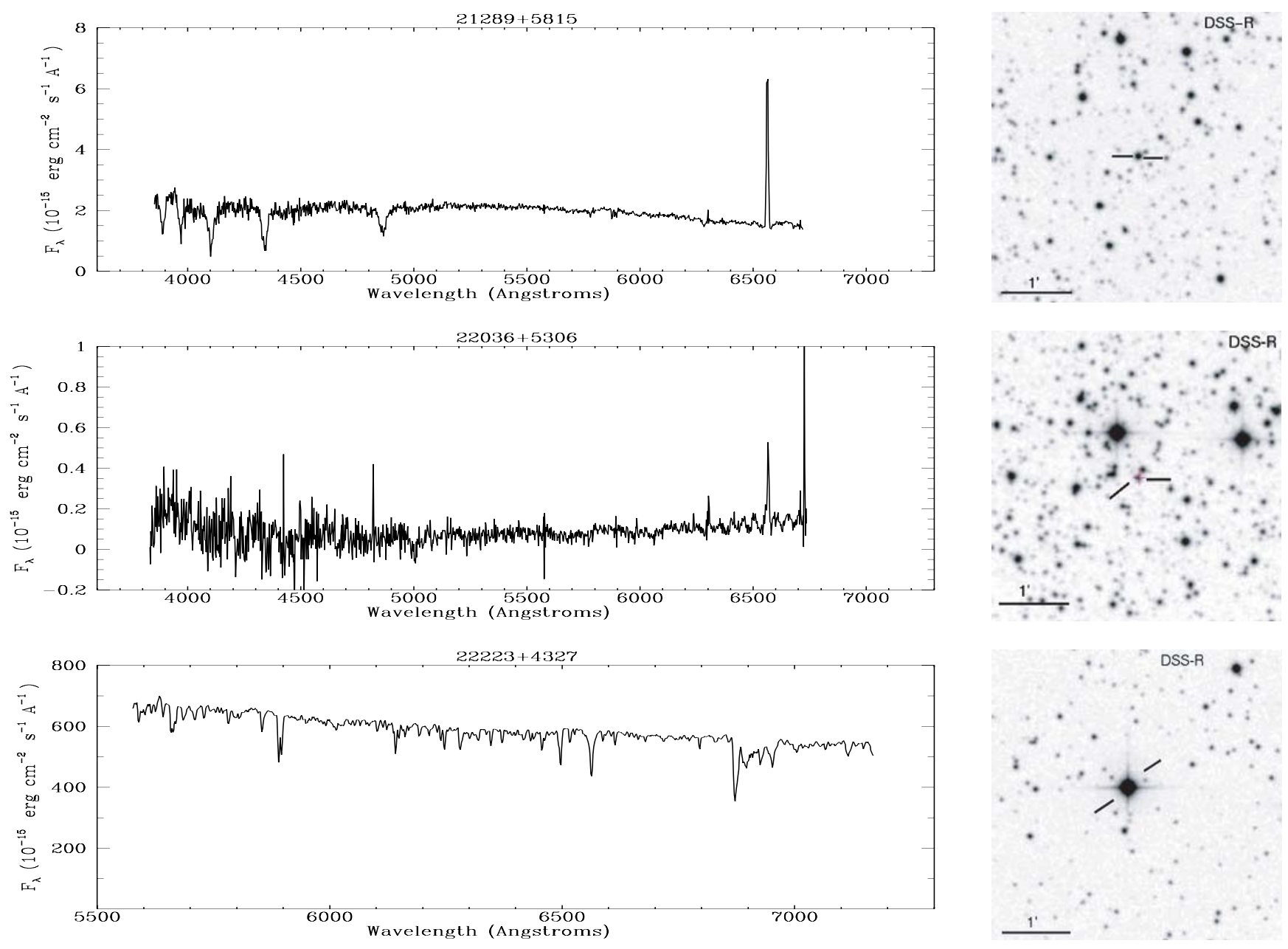

Fig. A.1. continued. 
O. Suárez et al.: Spectroscopic atlas of post-AGB and PNe, Online Material p 37

\section{Appendix B: Atlas of transition sources}
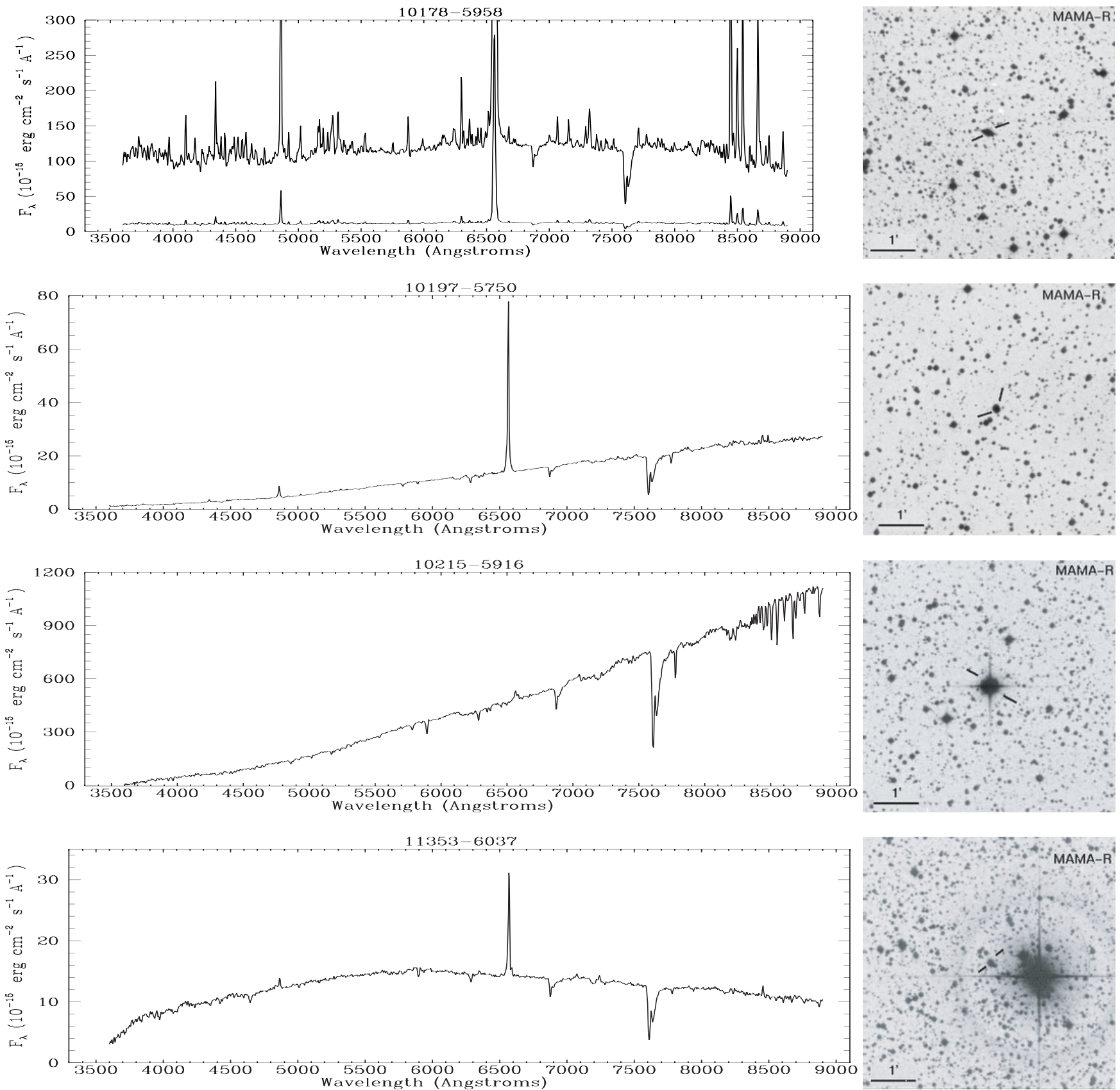

Fig. B.1. Spectra of the transition objects together with their corresponding identification charts. 
O. Suárez et al.: Spectroscopic atlas of post-AGB and PNe, Online Material p 38
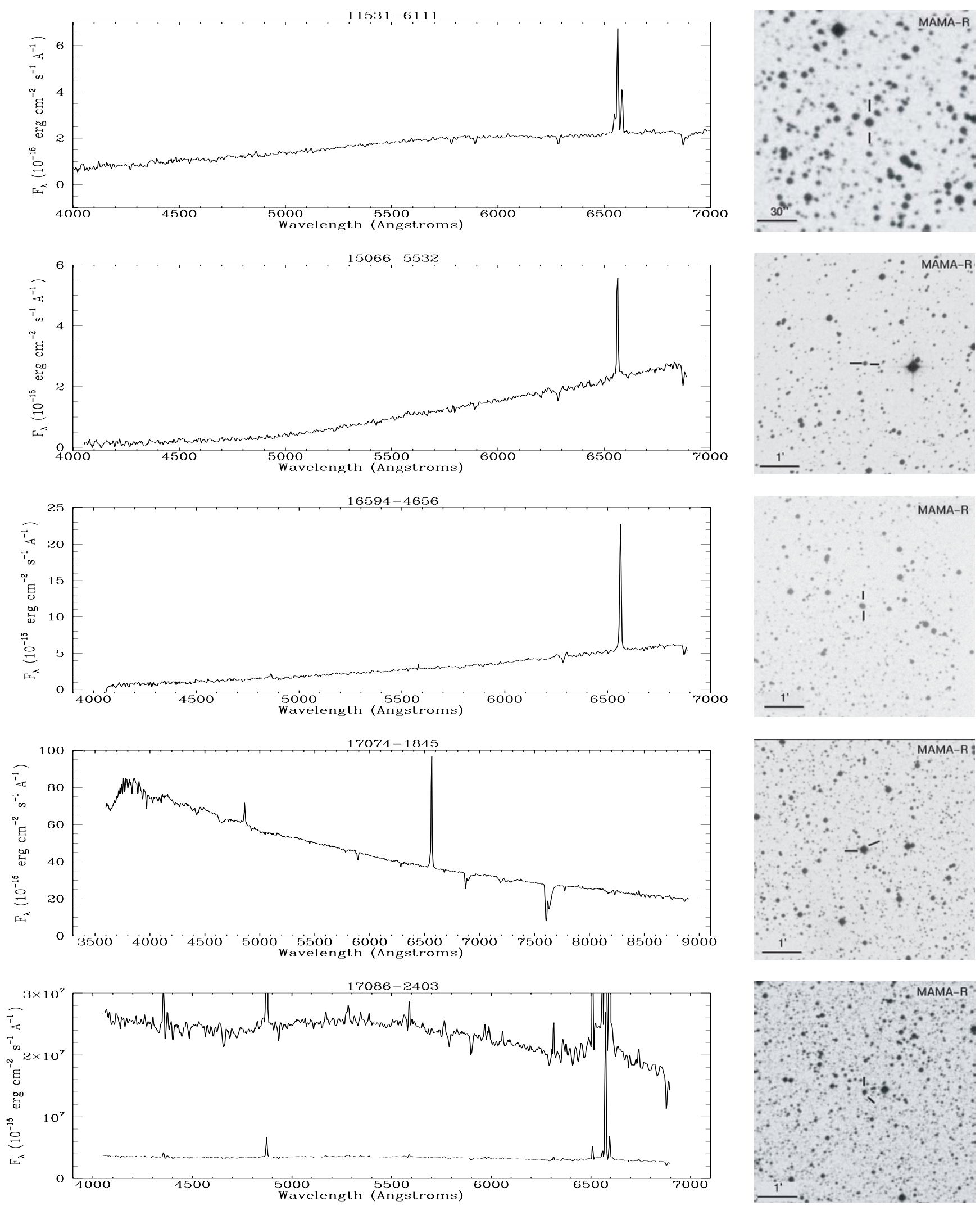

Fig. B.1. continued. 
O. Suárez et al.: Spectroscopic atlas of post-AGB and PNe, Online Material p 39
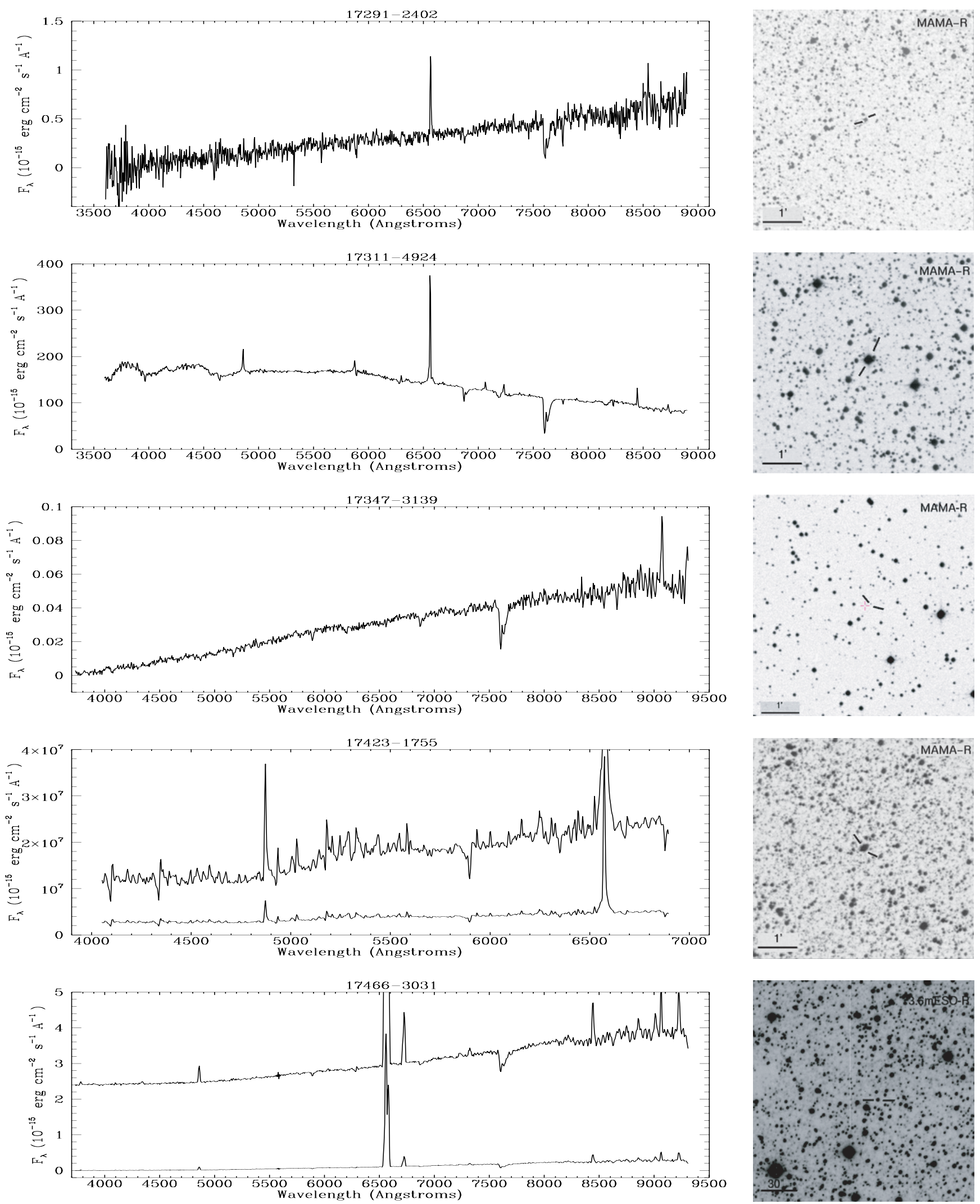

Fig. B.1. continued. 
O. Suárez et al.: Spectroscopic atlas of post-AGB and PNe, Online Material p 40
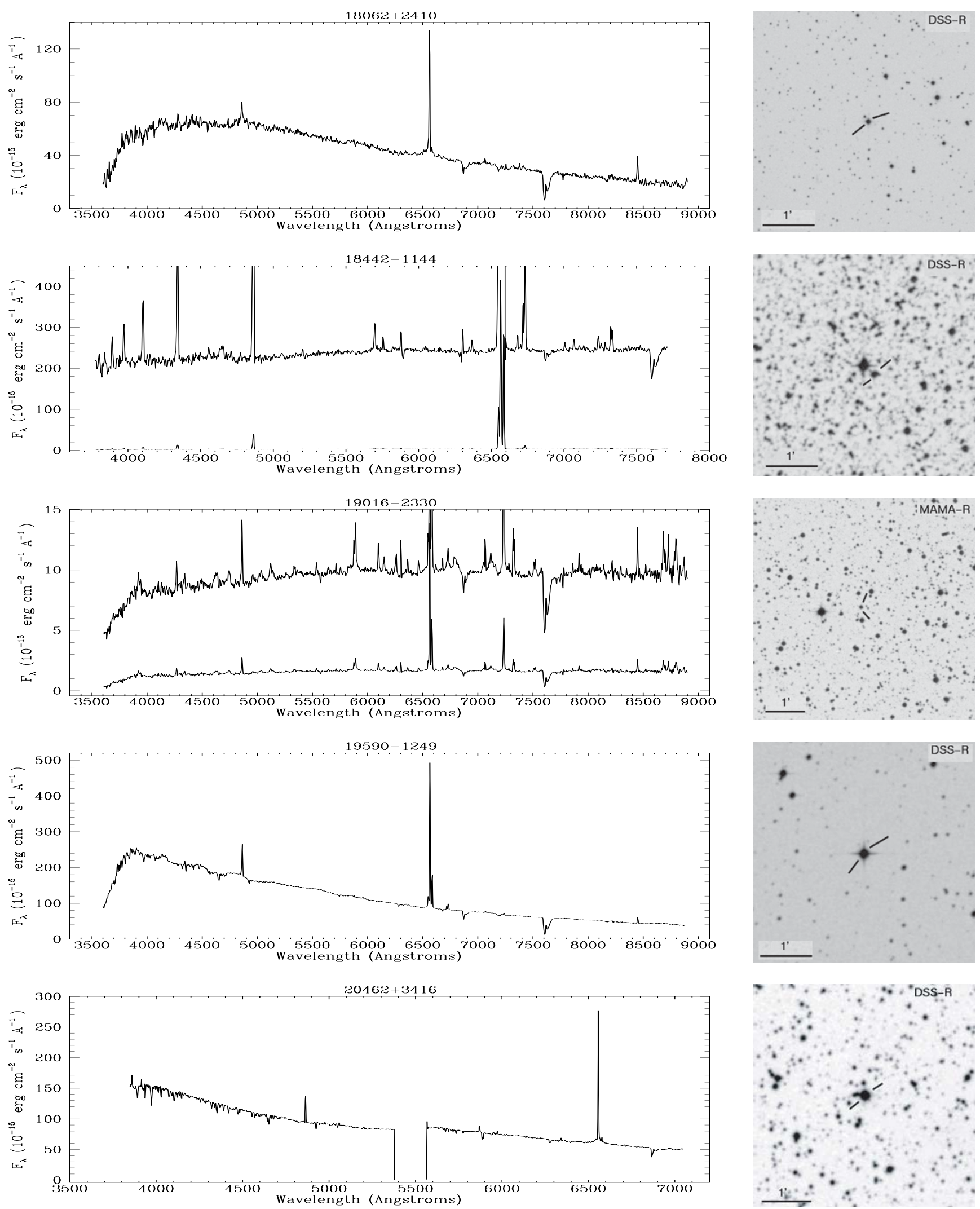

Fig. B.1. continued. 
O. Suárez et al.: Spectroscopic atlas of post-AGB and PNe, Online Material p 41
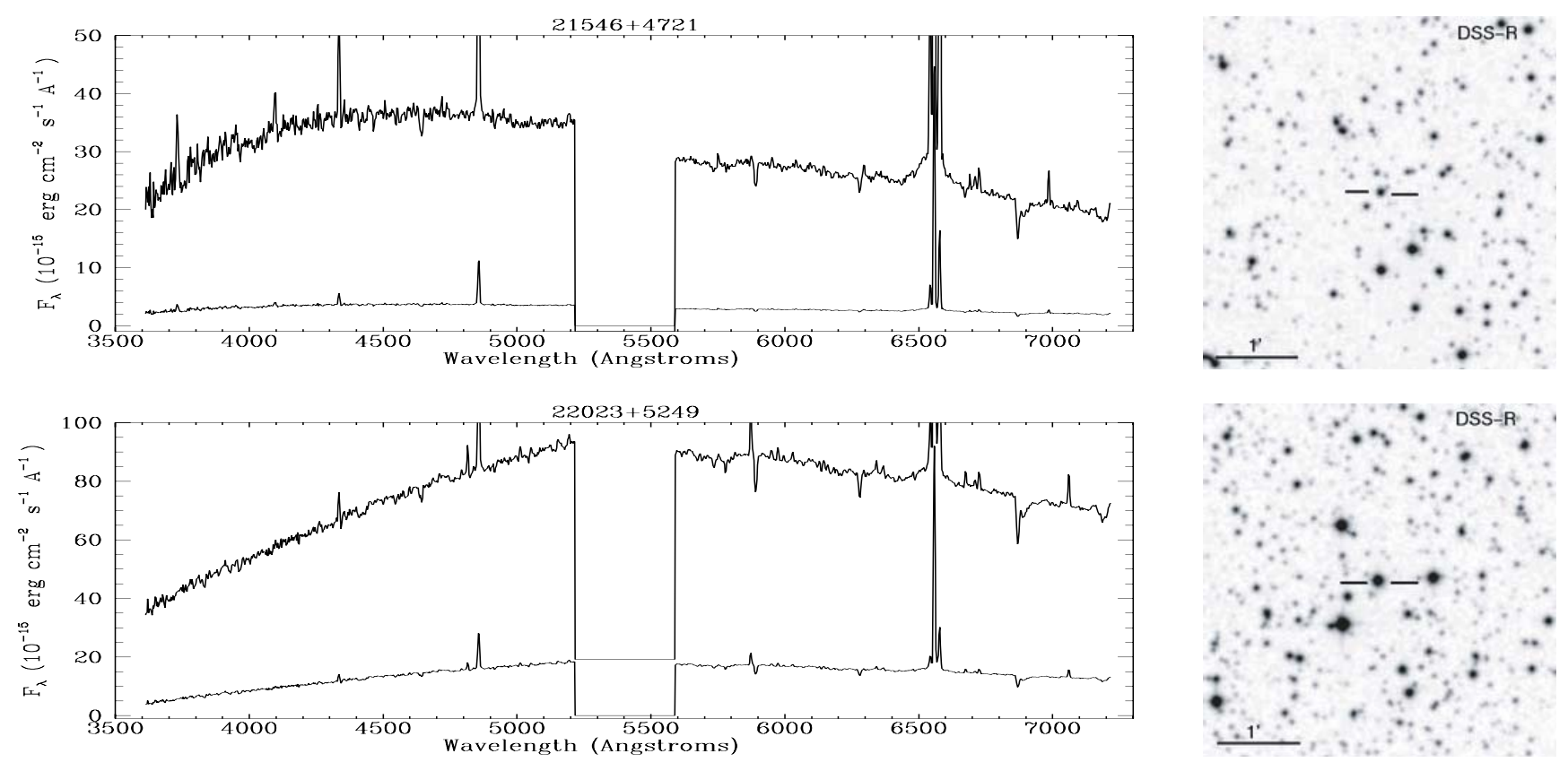

Fig. B.1. continued. 
O. Suárez et al.: Spectroscopic atlas of post-AGB and PNe, Online Material p 42

\section{Appendix C: Atlas of PNe}
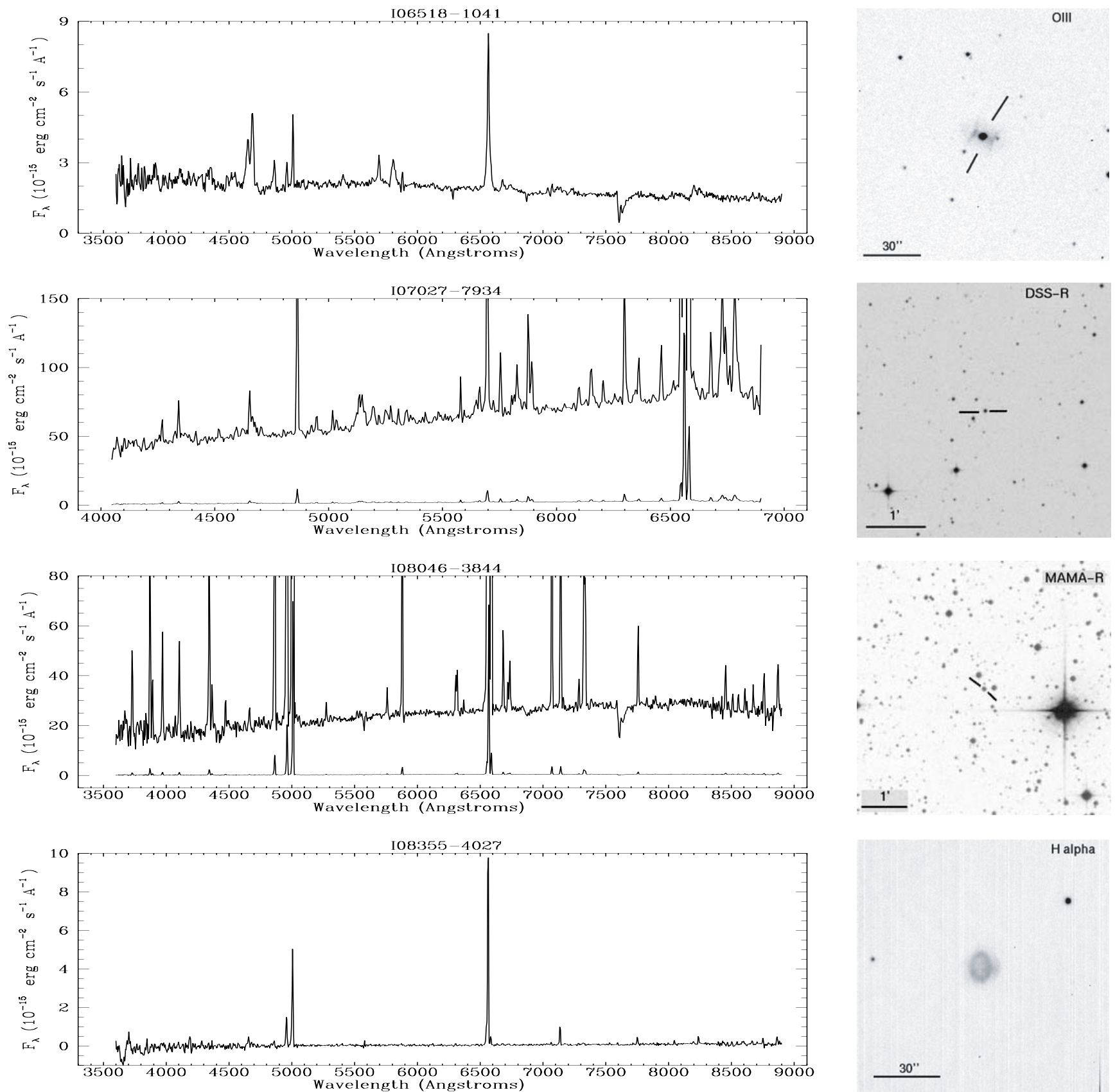

Fig. C.1. Spectra of the PNe in the sample together with their corresponding identification charts. 
O. Suárez et al.: Spectroscopic atlas of post-AGB and PNe, Online Material p 43
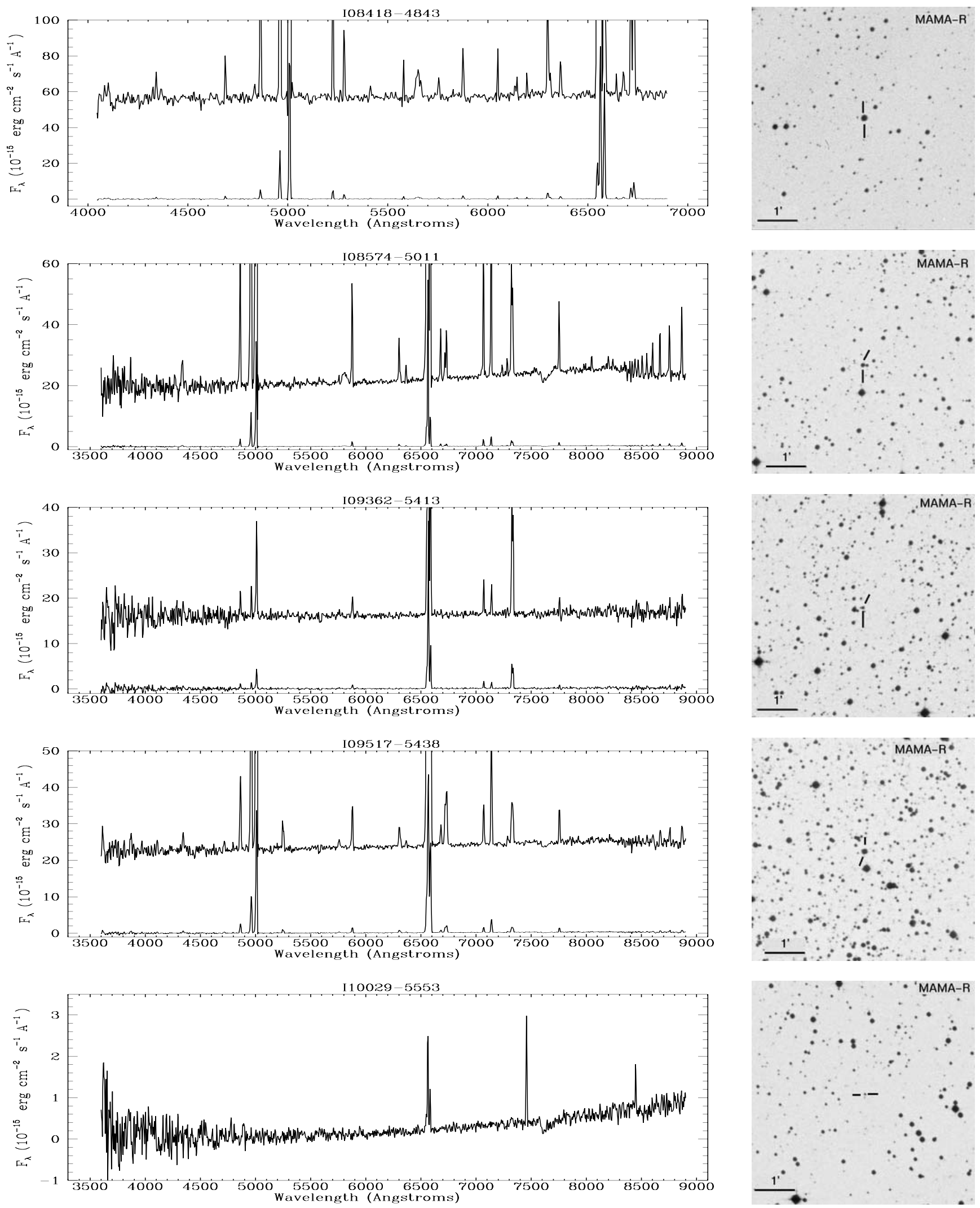

Fig. C.1. continued. 
O. Suárez et al.: Spectroscopic atlas of post-AGB and PNe, Online Material p 44
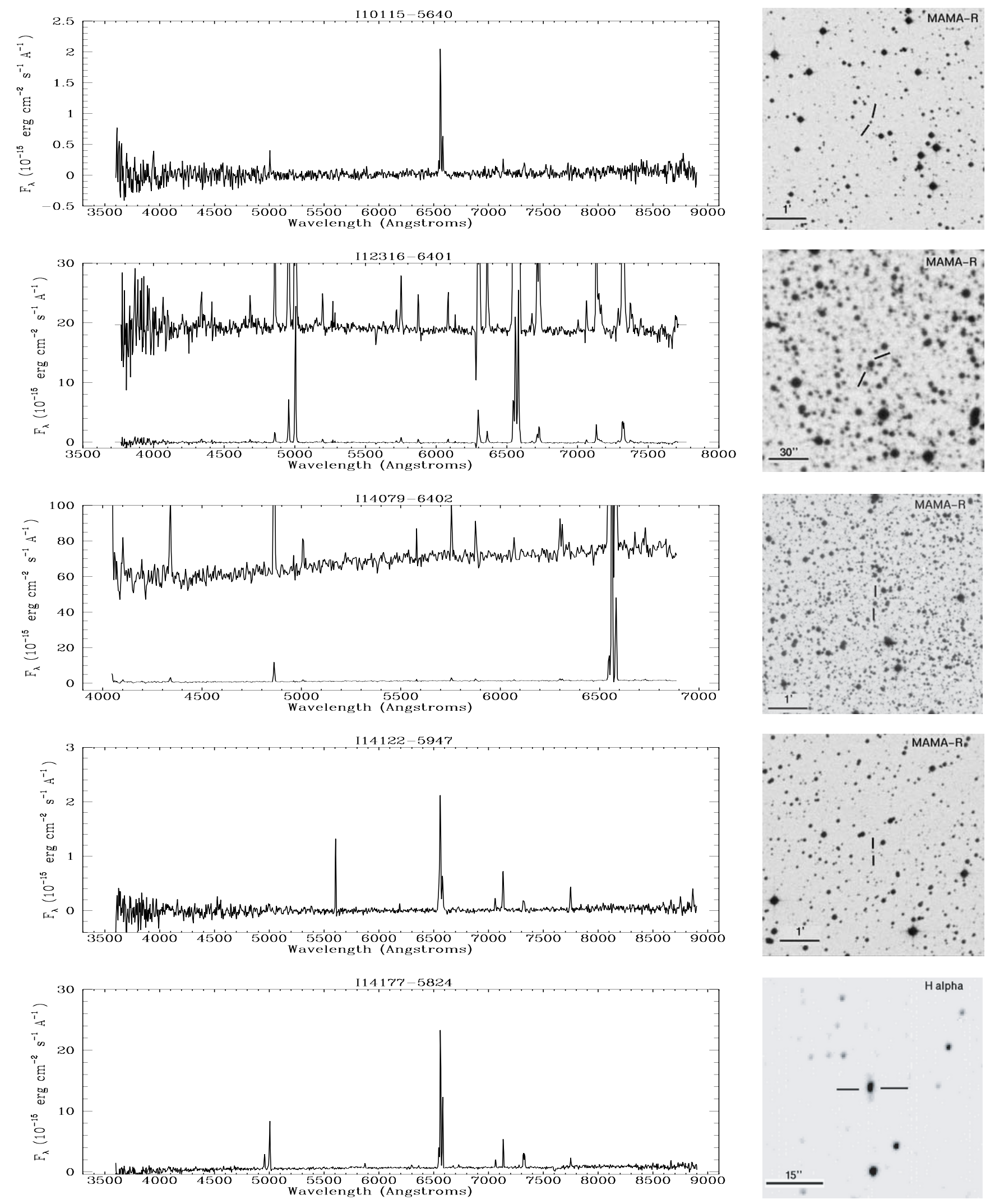

Fig. C.1. continued. 
O. Suárez et al.: Spectroscopic atlas of post-AGB and PNe, Online Material p 45
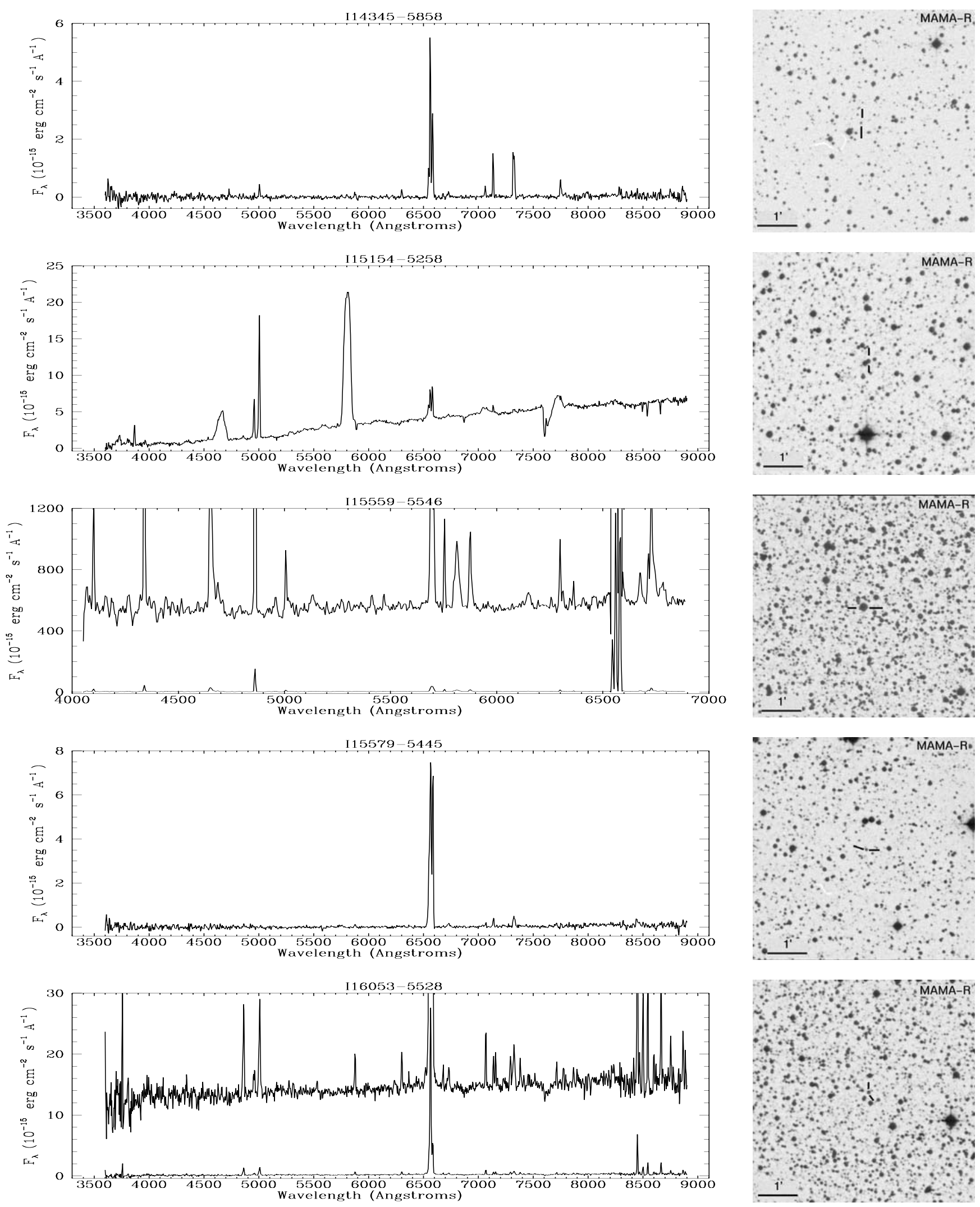

Fig. C.1. continued. 
O. Suárez et al.: Spectroscopic atlas of post-AGB and PNe, Online Material p 46
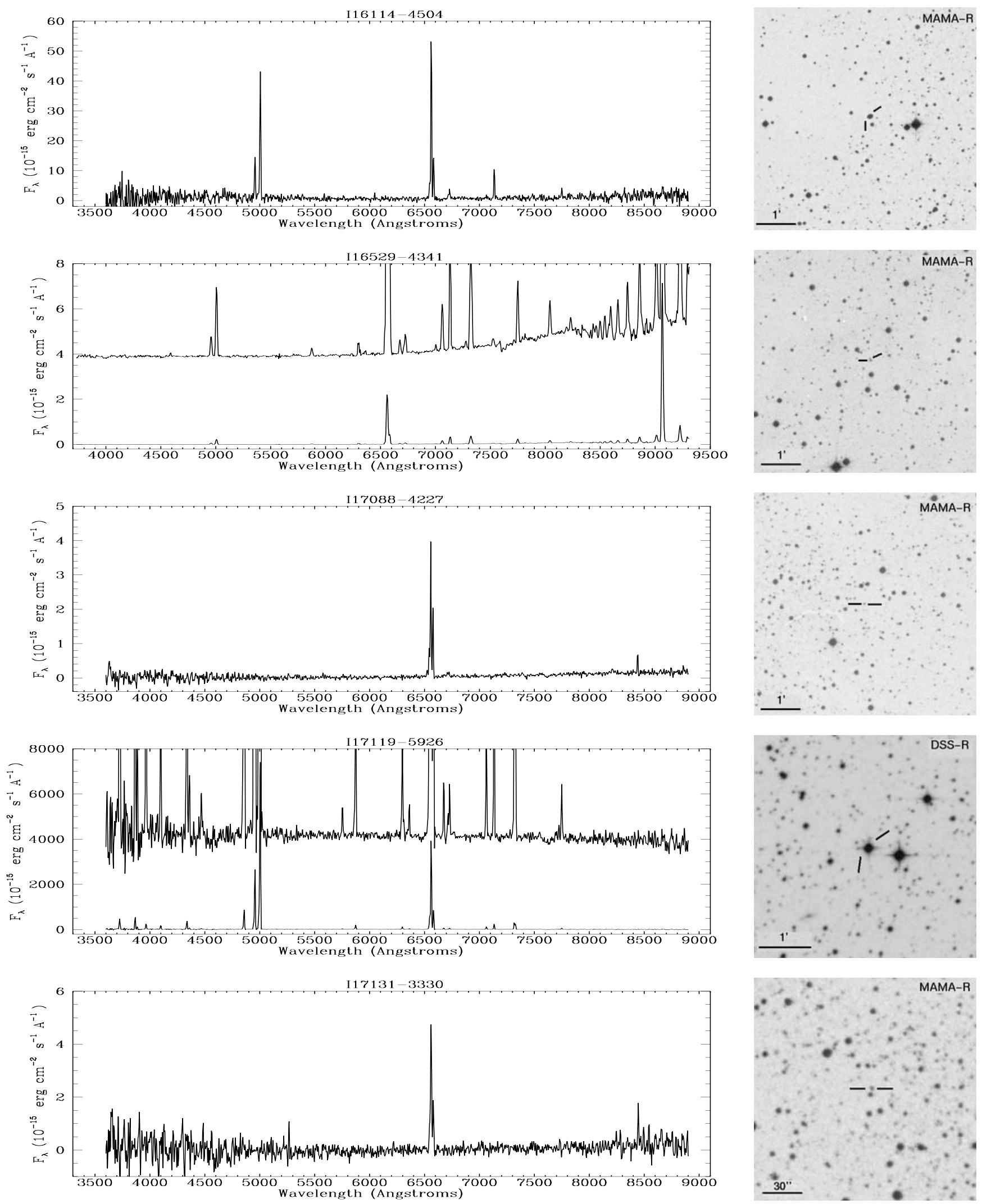

Fig. C.1. continued. 
O. Suárez et al.: Spectroscopic atlas of post-AGB and PNe, Online Material p 47
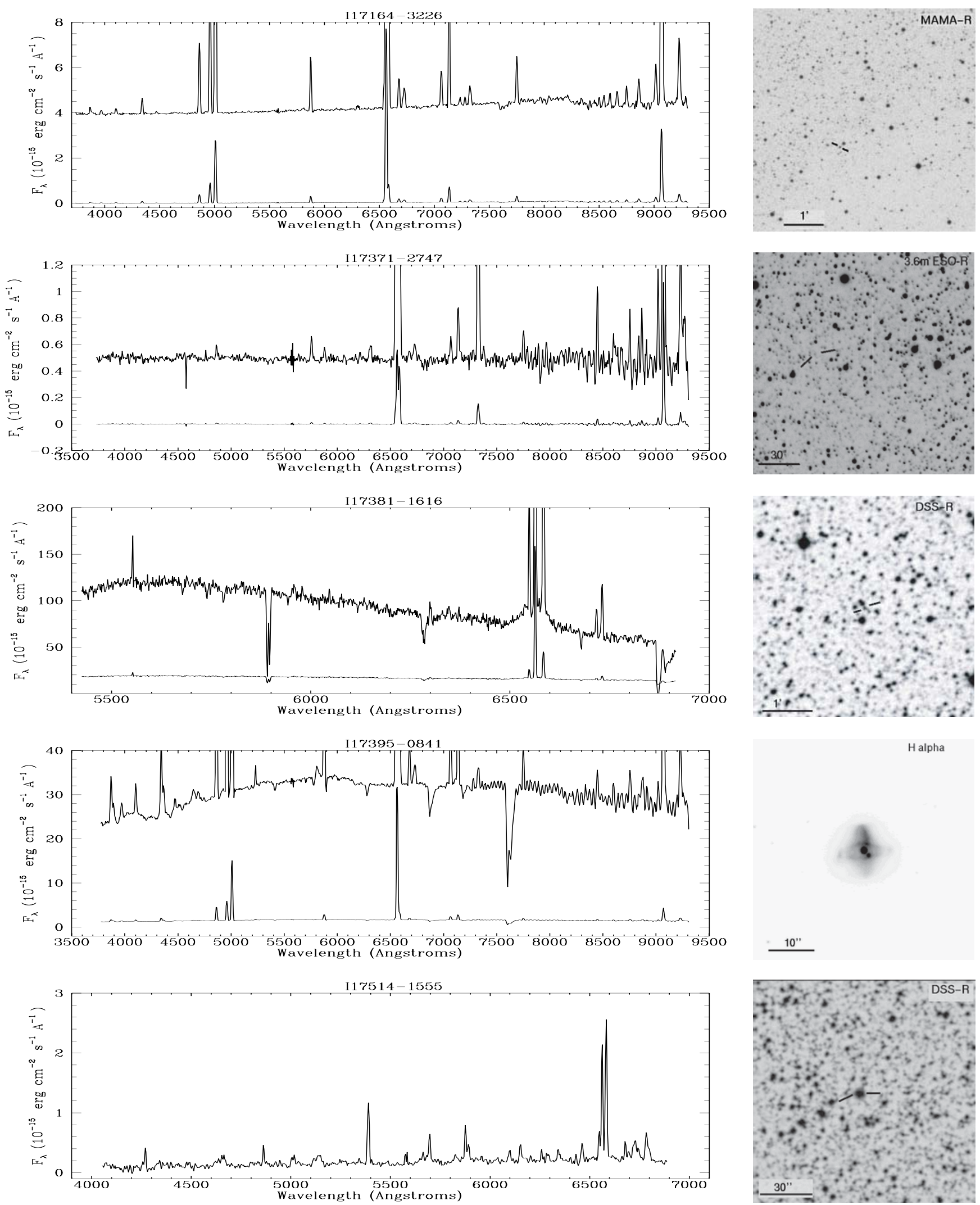

Fig. C.1. continued. 
O. Suárez et al.: Spectroscopic atlas of post-AGB and PNe, Online Material p 48
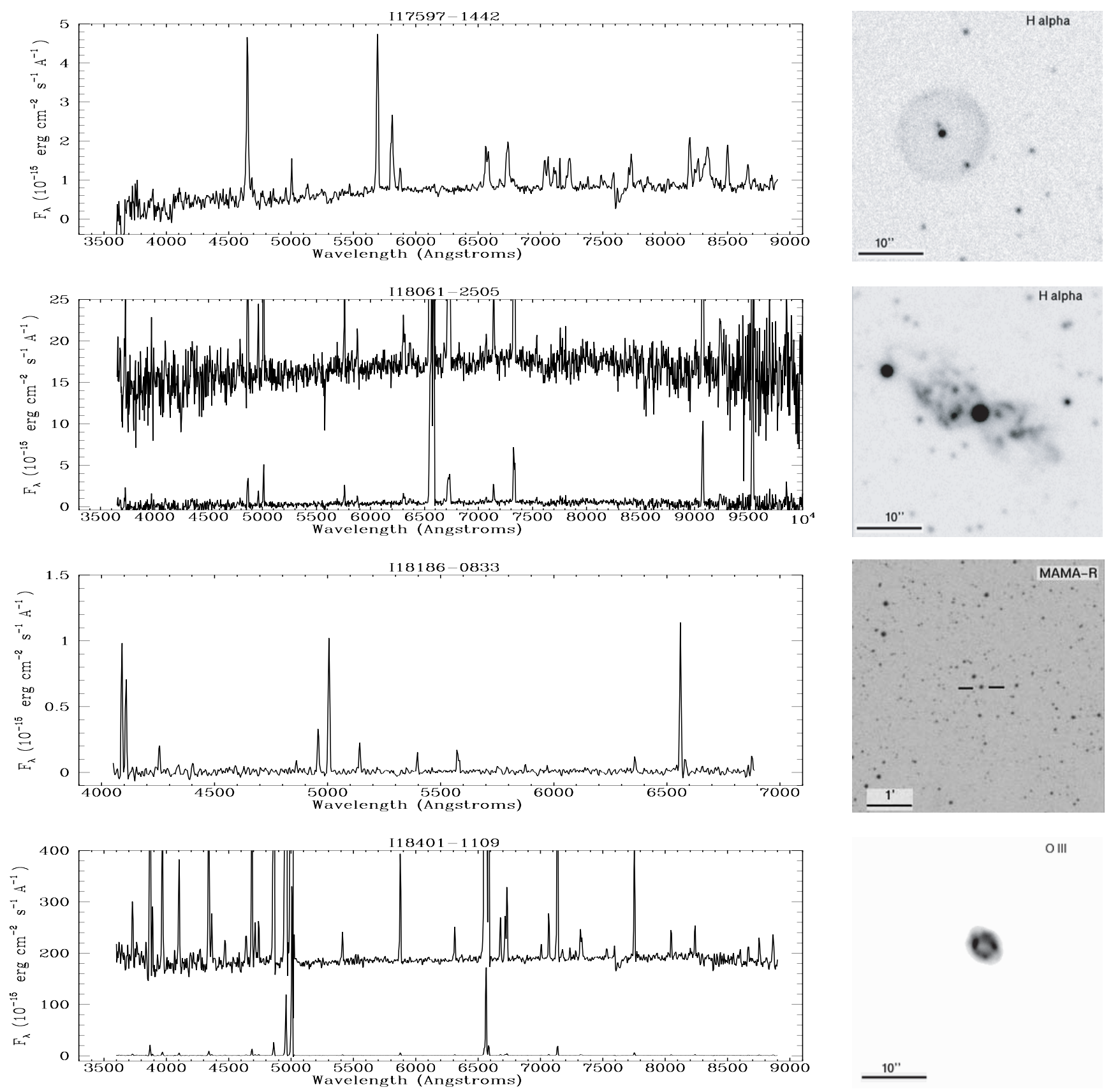

OIII
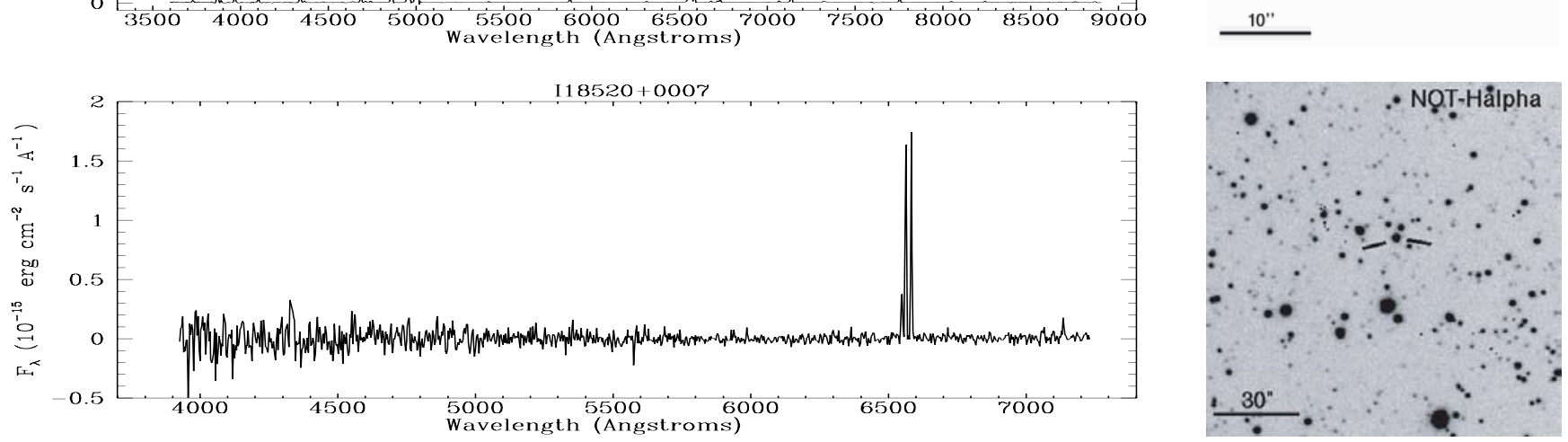

Fig. C.1. continued. 
O. Suárez et al.: Spectroscopic atlas of post-AGB and PNe, Online Material p 49
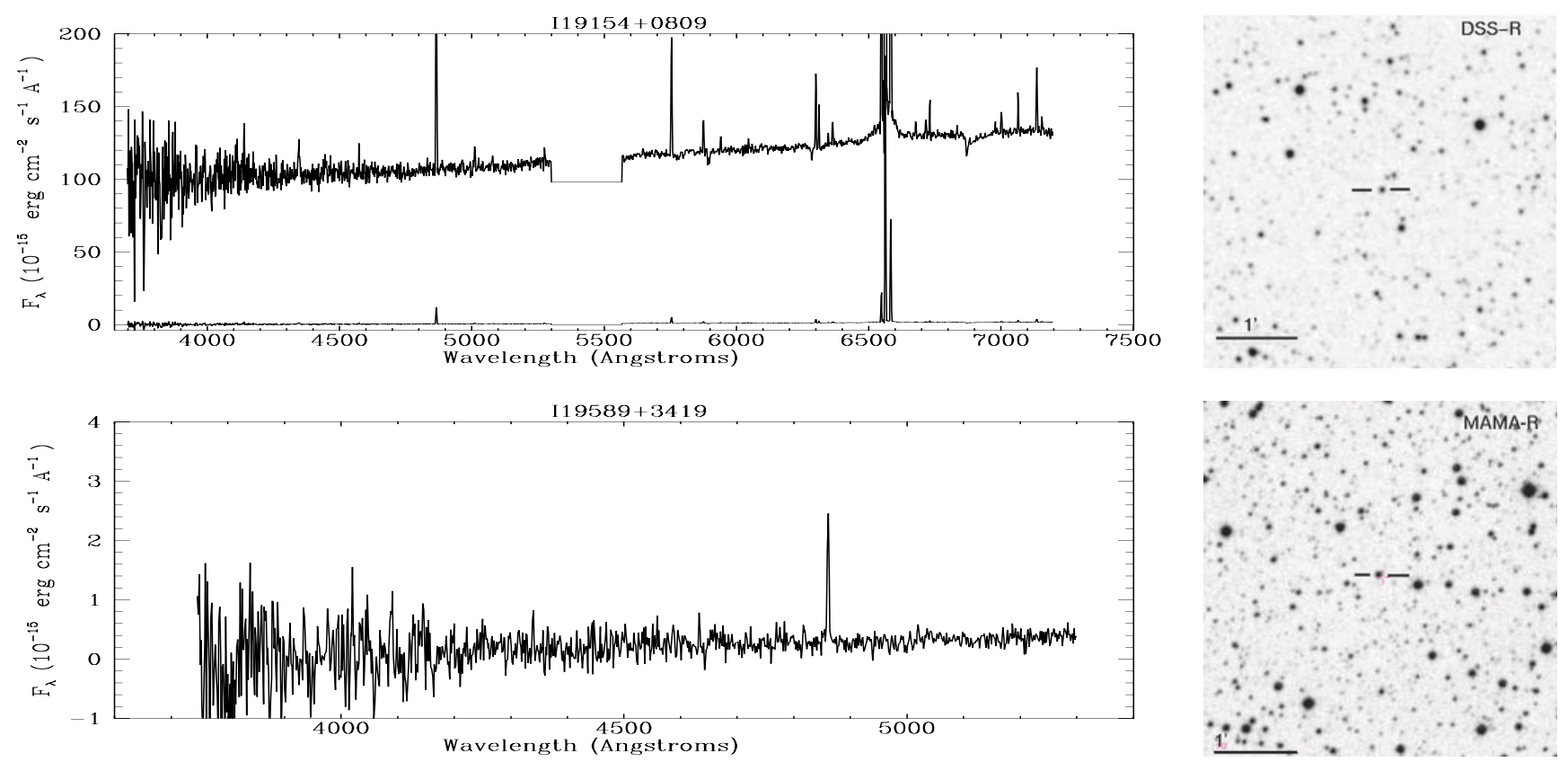

Fig. C.1. continued. 


\section{Appendix D: Atlas of young stellar objects}
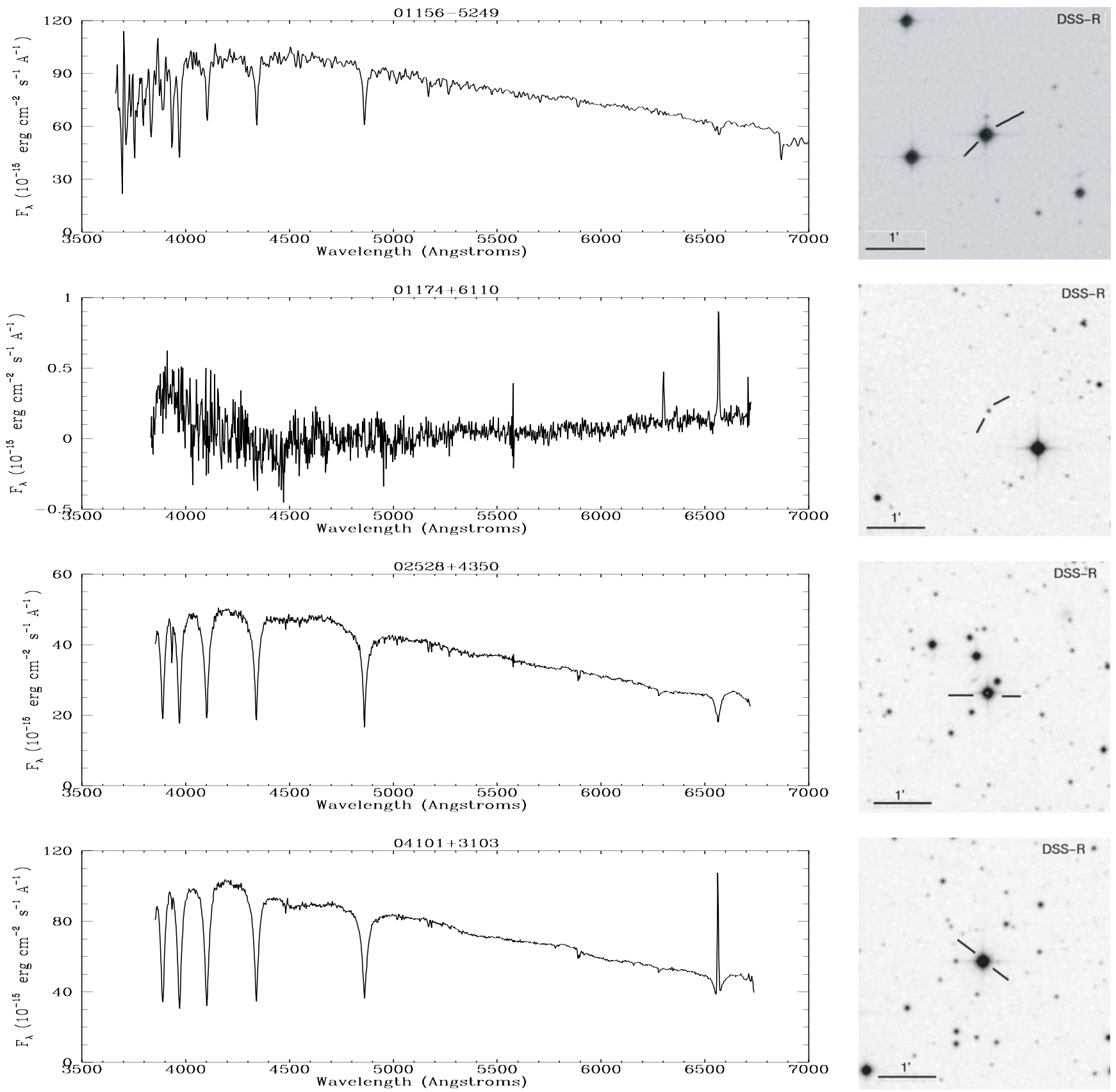

Fig. D.1. Spectra of the objects classified as young stars in the sample together with their corresponding identification charts. 
O. Suárez et al.: Spectroscopic atlas of post-AGB and PNe, Online Material p 51
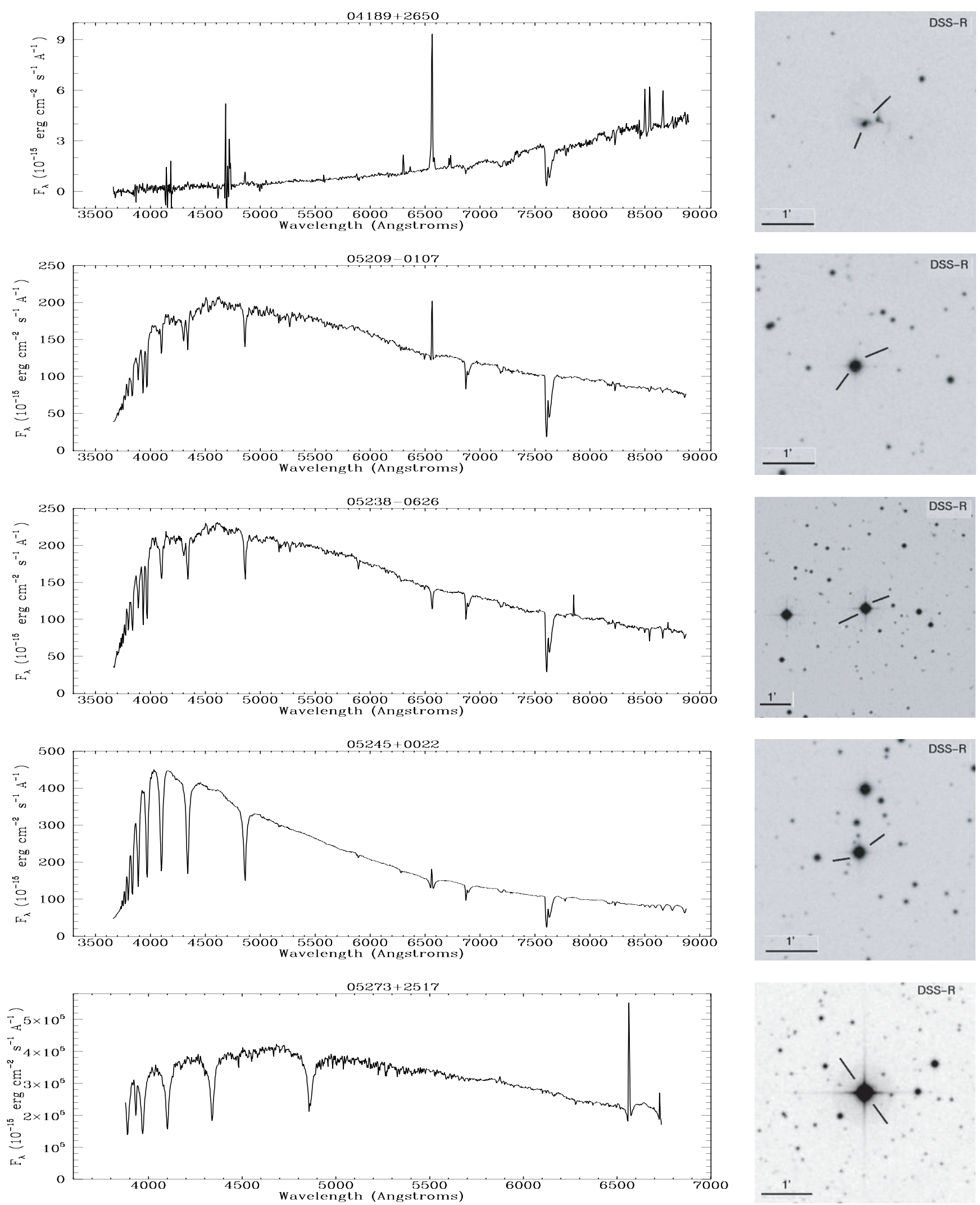

Fig. D.1. continued. 
O. Suárez et al.: Spectroscopic atlas of post-AGB and PNe, Online Material p 52
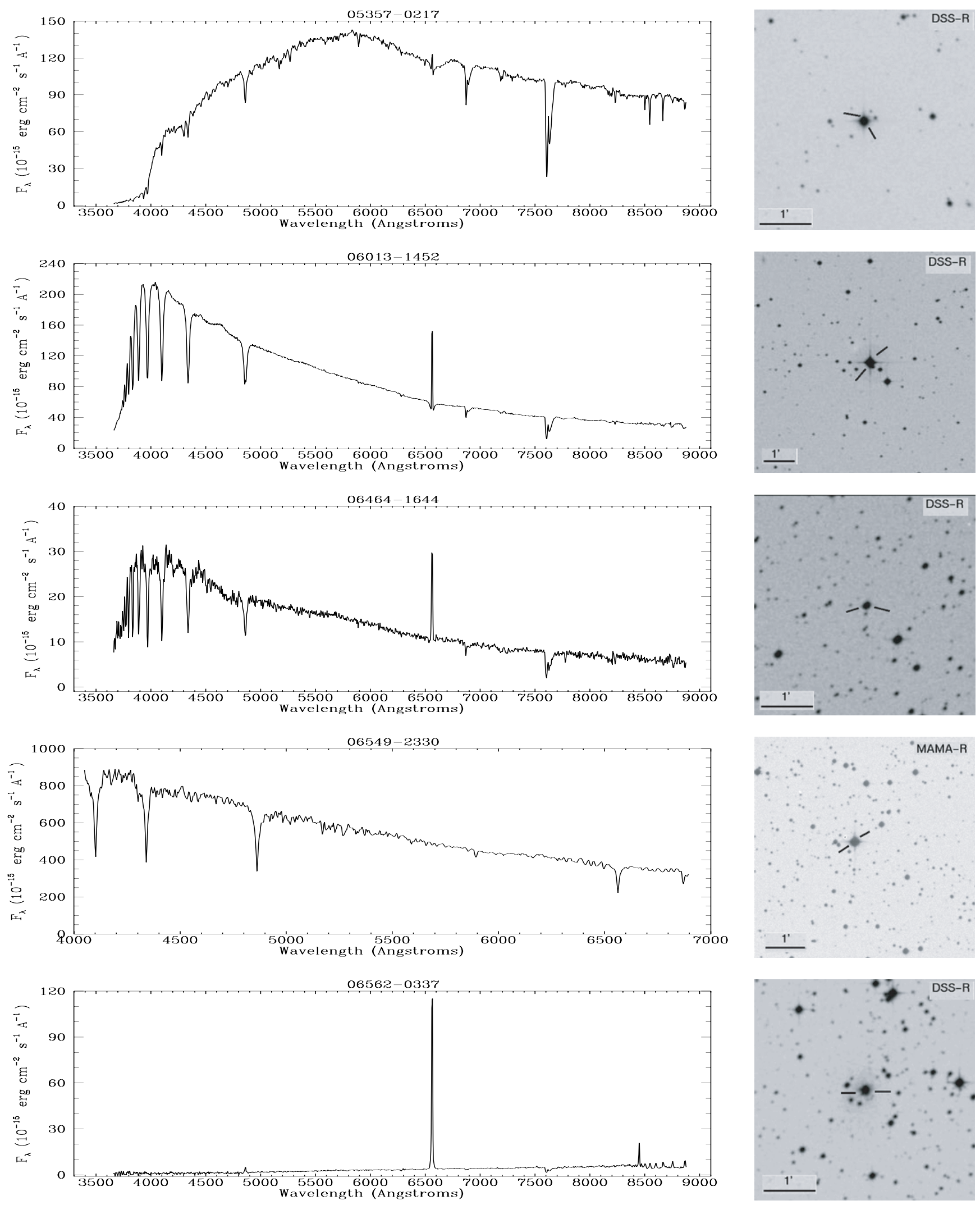

Fig. D.1. continued. 
O. Suárez et al.: Spectroscopic atlas of post-AGB and PNe, Online Material p 53
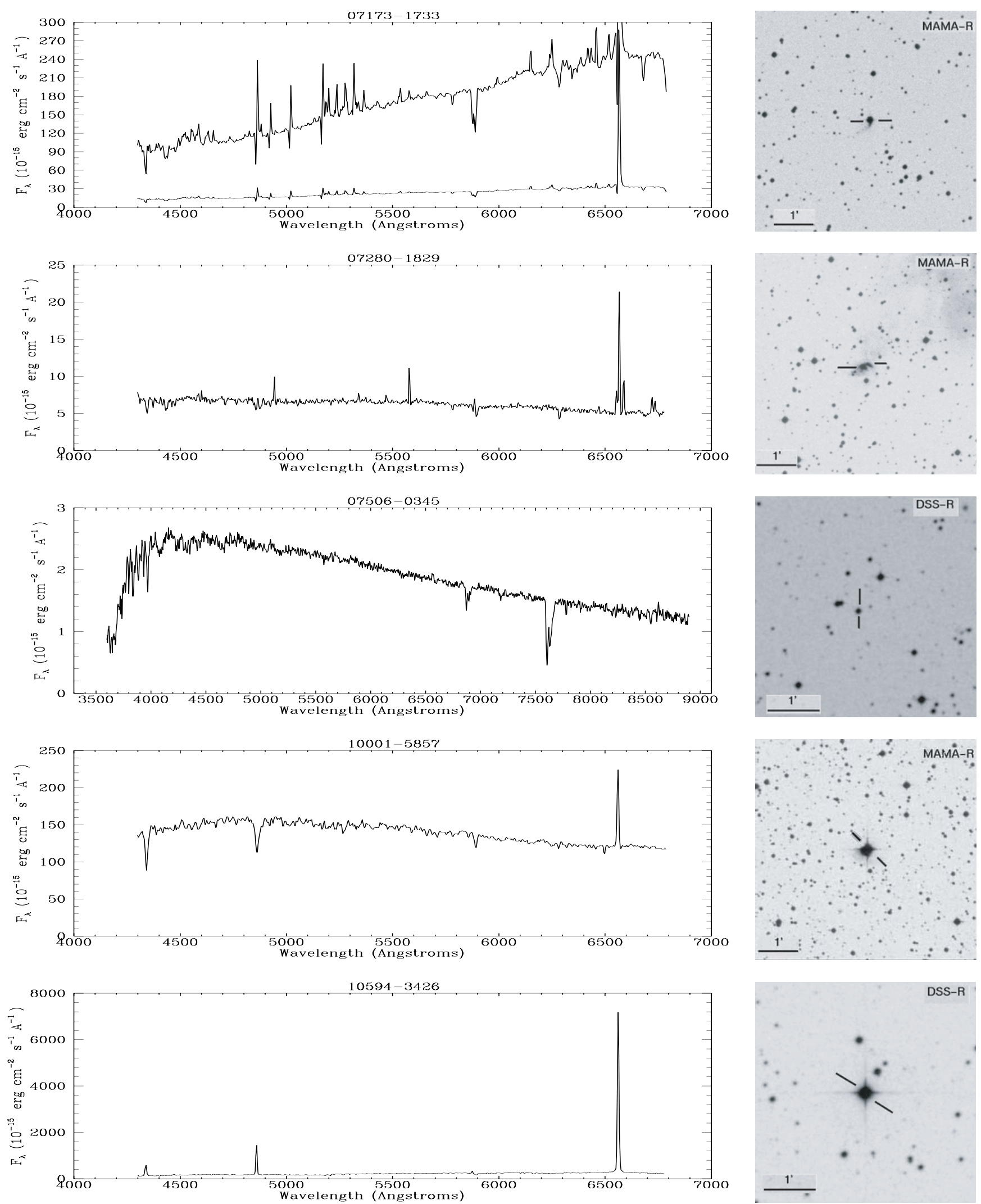

Fig. D.1. continued. 
O. Suárez et al.: Spectroscopic atlas of post-AGB and PNe, Online Material p 54
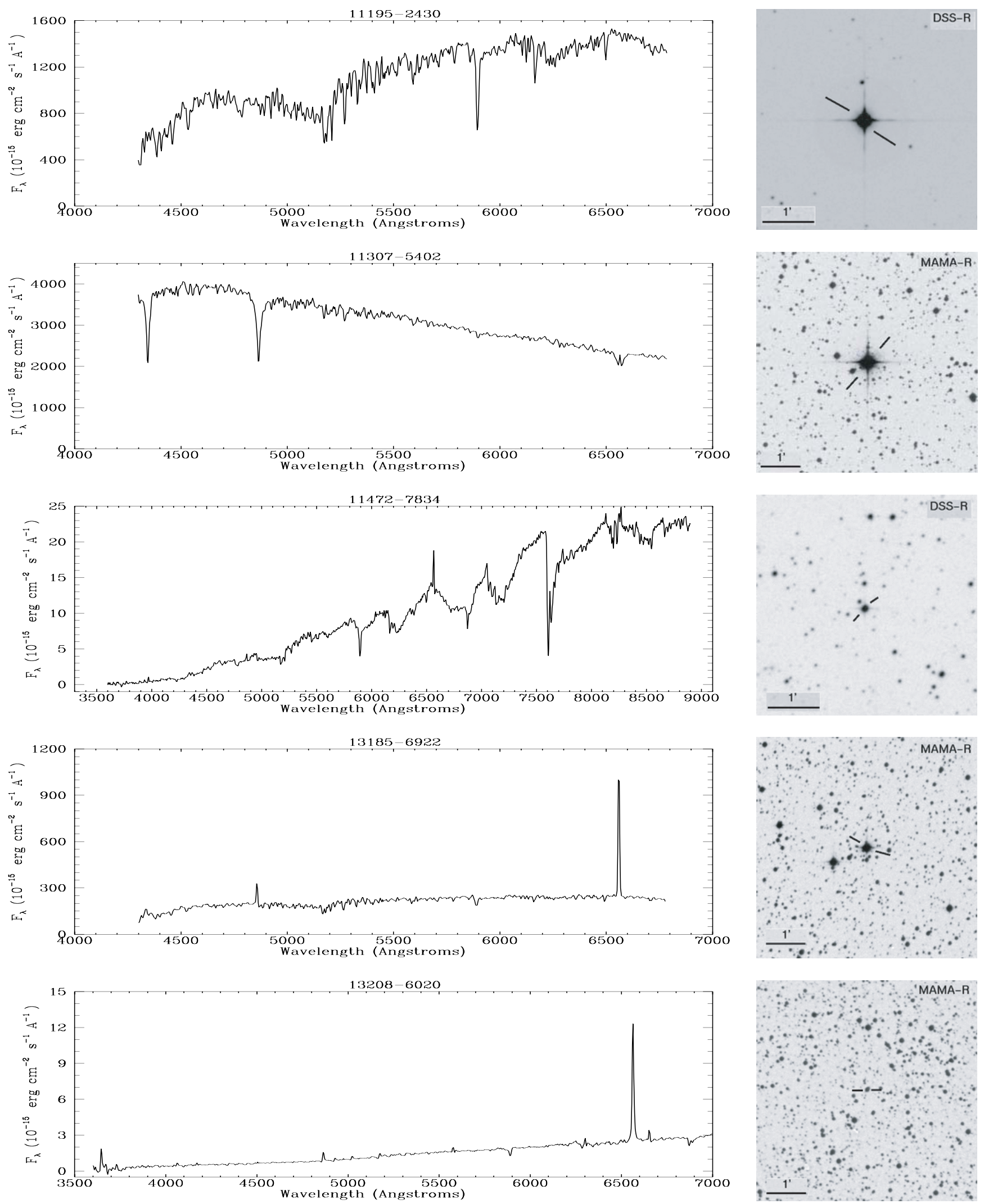

Fig. D.1. continued. 
O. Suárez et al.: Spectroscopic atlas of post-AGB and PNe, Online Material p 55
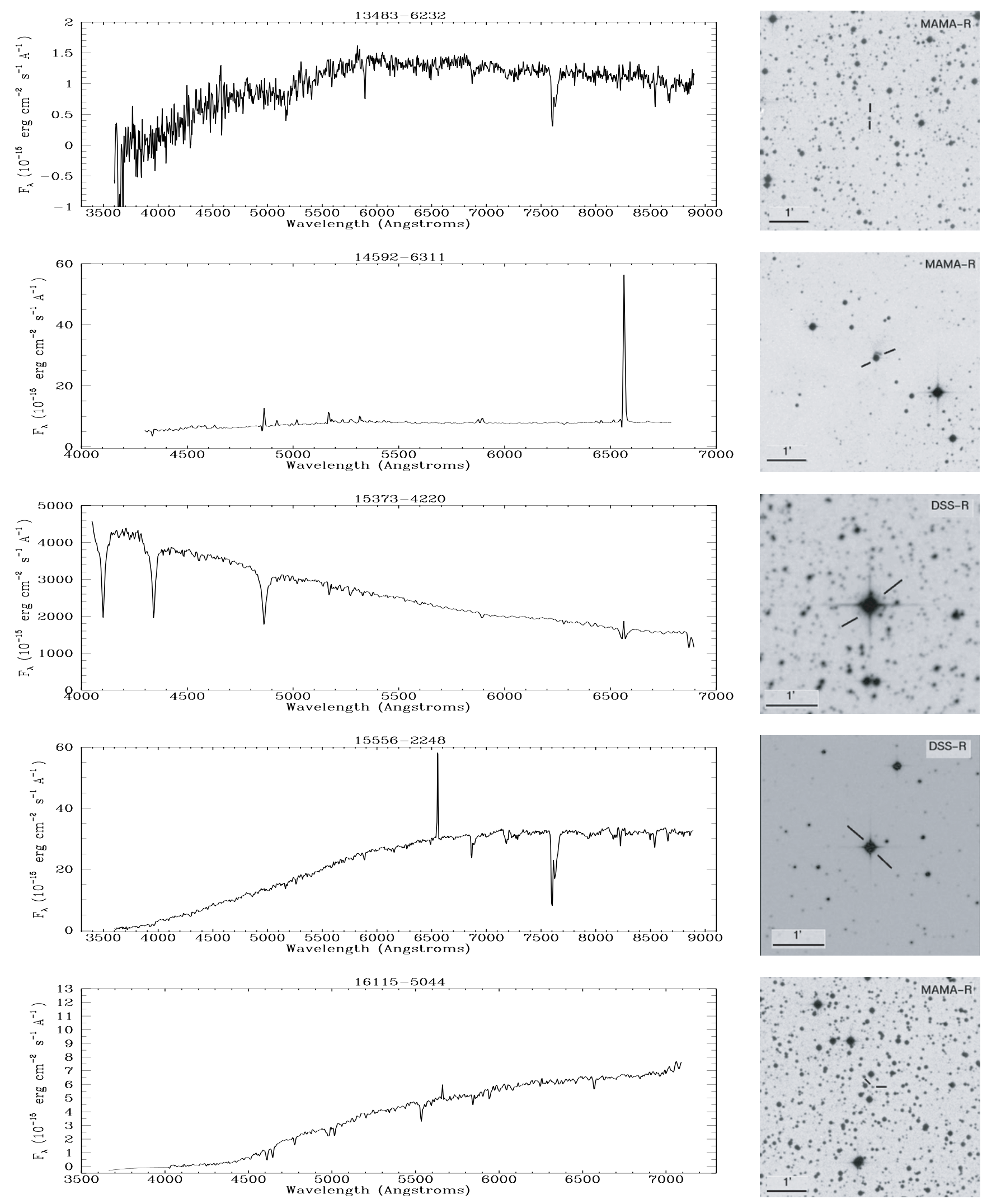

Fig. D.1. continued. 
O. Suárez et al.: Spectroscopic atlas of post-AGB and PNe, Online Material p 56
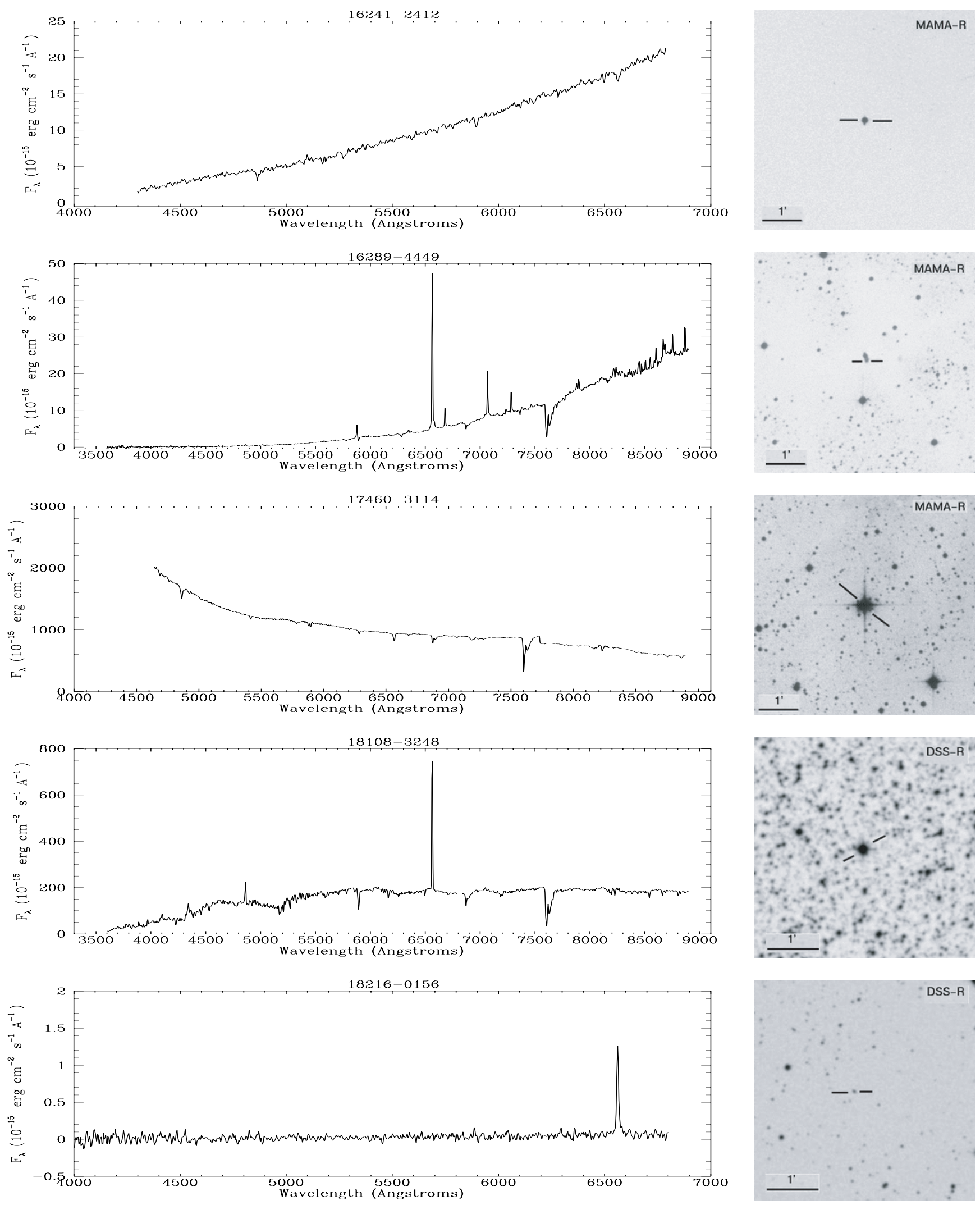

Fig. D.1. continued. 
O. Suárez et al.: Spectroscopic atlas of post-AGB and PNe, Online Material p 57
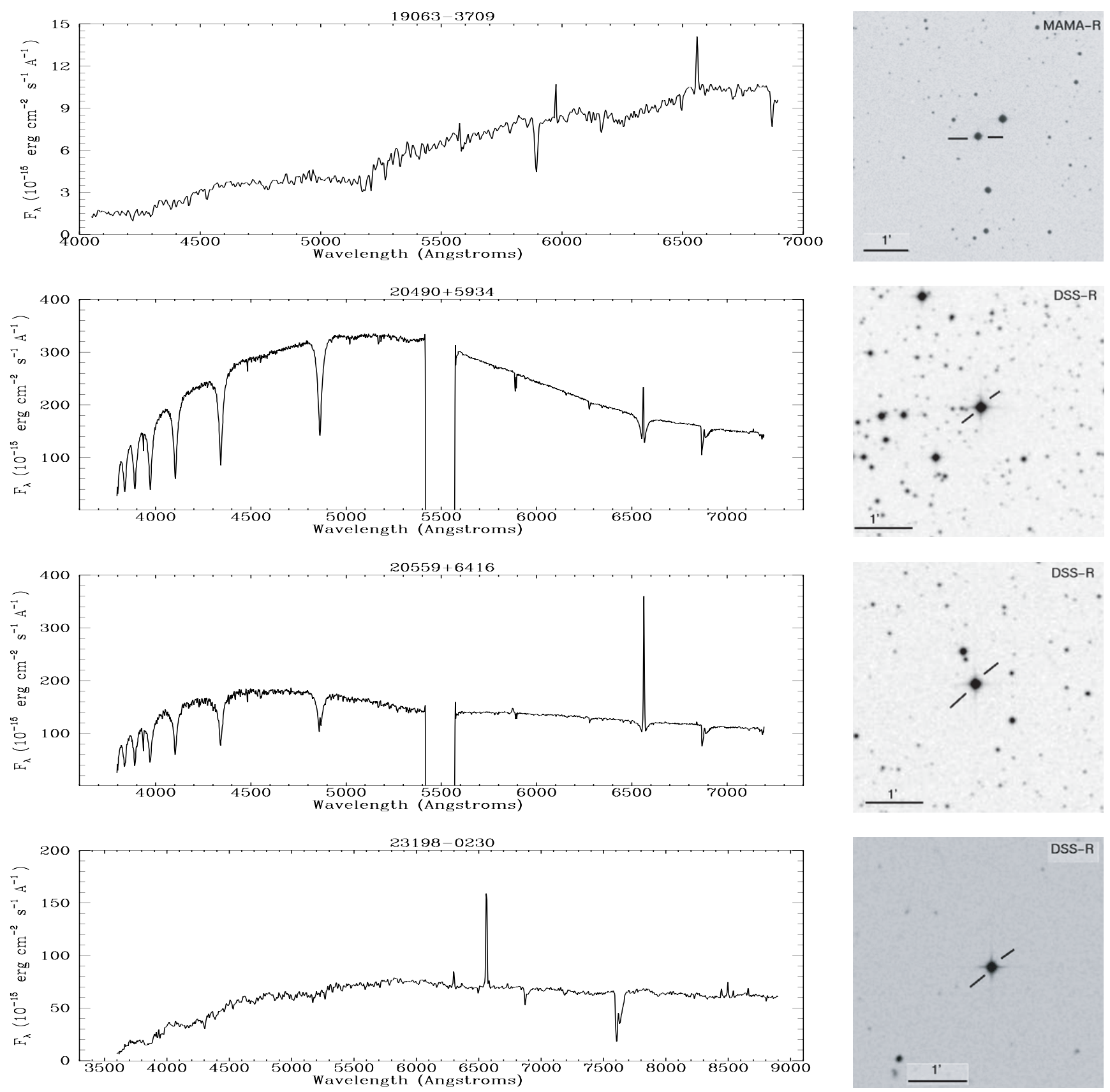

Fig. D.1. continued. 
O. Suárez et al.: Spectroscopic atlas of post-AGB and PNe, Online Material p 58

\section{Appendix E: Atlas of galaxies}
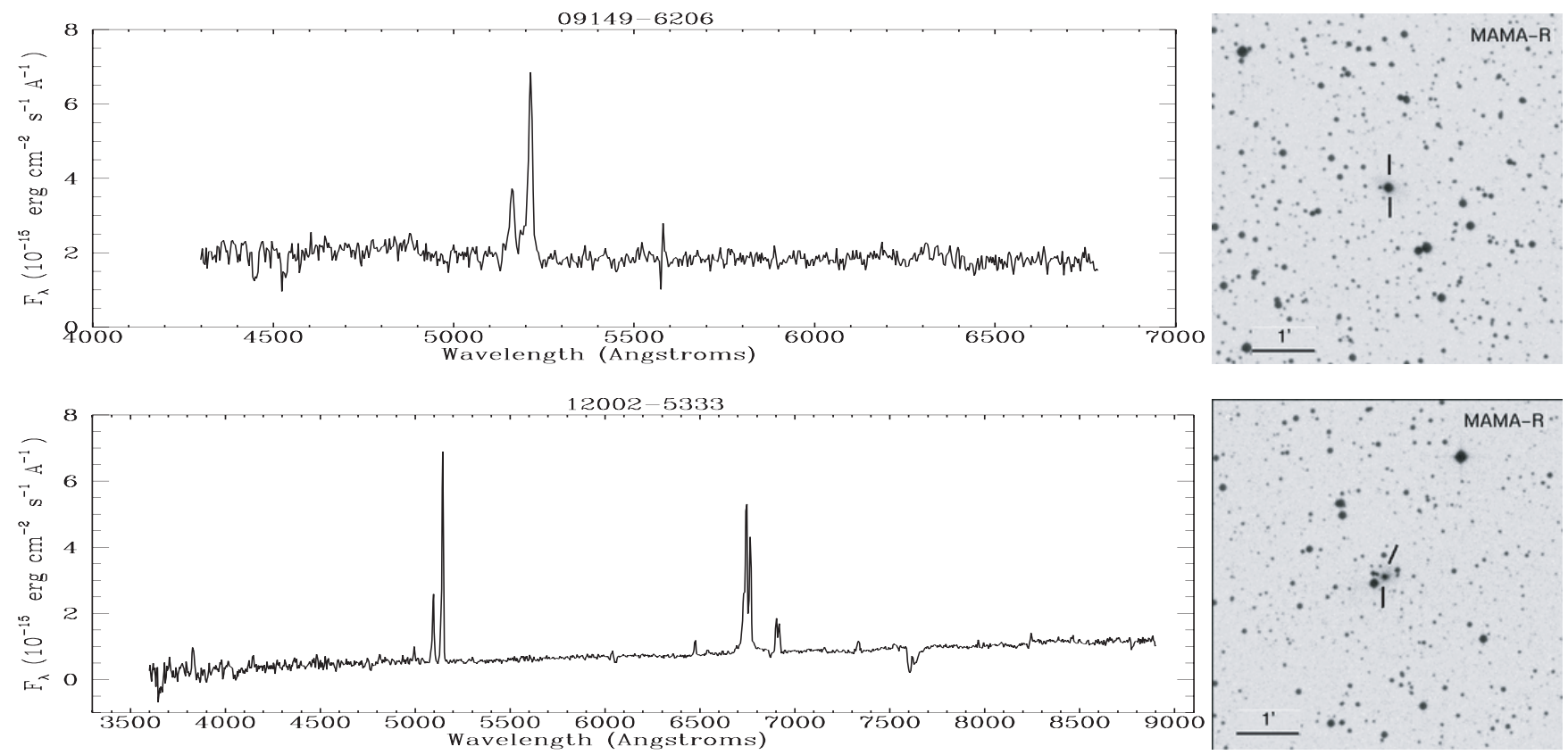

Fig. E.1. Spectra of the sources classified as galaxies in the sample together with their corresponding identification charts. 
O. Suárez et al.: Spectroscopic atlas of post-AGB and PNe, Online Material p 59

\section{Appendix F: Atlas of peculiar sources}
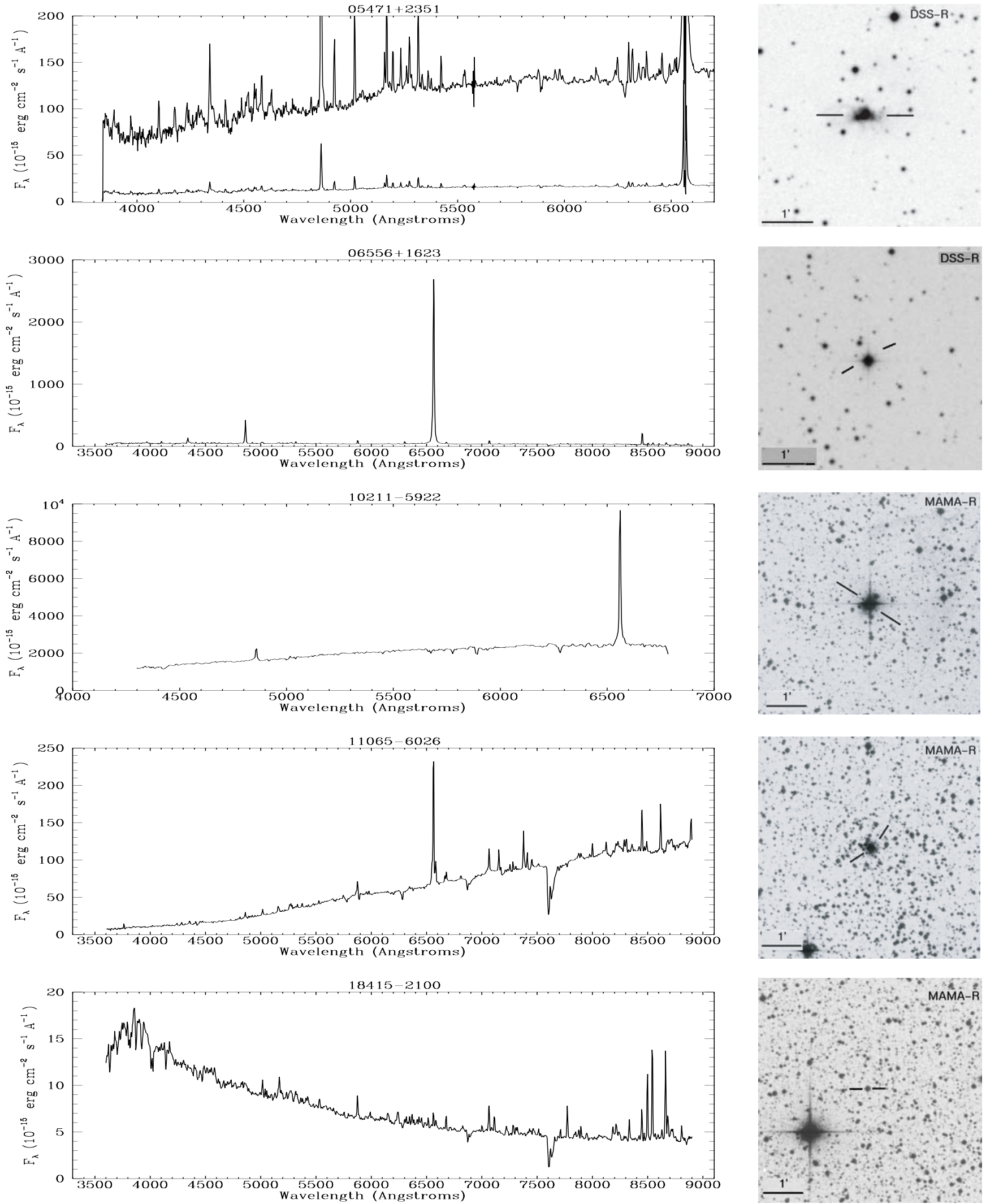

Fig. F.1. Spectra of the peculiar sources in the sample together with their corresponding identification charts. 